\title{
The Galois theory of matrix C-rings.
}

\section{Turner, Ryan}

How to cite:

Turner, Ryan (2006) The Galois theory of matrix C-rings.. thesis, Swansea University.

http://cronfa.swan.ac.uk/Record/cronfa42607

Use policy:

This item is brought to you by Swansea University. Any person downloading material is agreeing to abide by the terms of the repository licence: copies of full text items may be used or reproduced in any format or medium, without prior permission for personal research or study, educational or non-commercial purposes only. The copyright for any work remains with the original author unless otherwise specified. The full-text must not be sold in any format or medium without the formal permission of the copyright holder. Permission for multiple reproductions should be obtained from the original author.

Authors are personally responsible for adhering to copyright and publisher restrictions when uploading content to the repository.

Please link to the metadata record in the Swansea University repository, Cronfa (link given in the citation reference above.)

http://www.swansea.ac.uk/library/researchsupport/ris-support/ 


\title{
The Galois Theory of Matrix $C$-rings
}

\author{
Ryan Turner
}

Submitted to the University of Wales in fulfillment of the requirements for the Degree of Doctor of Philosophy

Department of Mathematics

University of Wales Swansea

September 2006 
ProQuest Number: 10805365

All rights reserved

INFORMATION TO ALL USERS

The quality of this reproduction is dependent upon the quality of the copy submitted.

In the unlikely event that the author did not send a complete manuscript and there are missing pages, these will be noted. Also, if material had to be removed, a note will indicate the deletion.

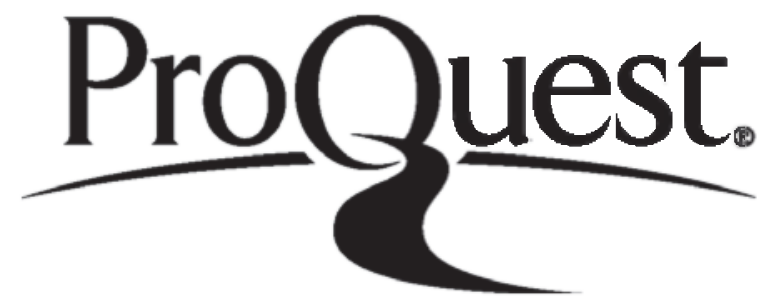

ProQuest 10805365

Published by ProQuest LLC (2018). Copyright of the Dissertation is held by the Author.

All rights reserved.

This work is protected against unauthorized copying under Title 17, United States Code Microform Edition (C) ProQuest LLC.

ProQuest LLC.

789 East Eisenhower Parkway

P.O. Box 1346

Ann Arbor, Ml $48106-1346$ 


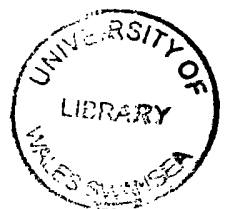




\section{Declaration}

This work has not previously been accepted in substance for any degree and is not being concurrently submitted in candidature for any degree.

Signed.

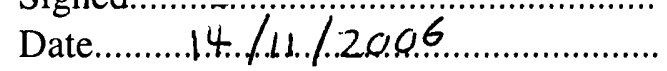

\section{Statement 1}

This thesis is the result of my own investigations, except where otherwise indicated. Other sources are acknowledged by references and a bibliography is appended.

Signed

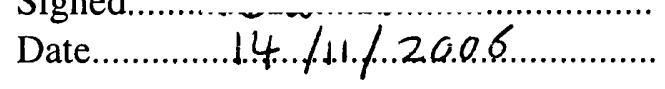

\section{Statement 2}

I hereby give consent for my thesis, if accepted, to be available for photocopying and for inter-library loan, and for the title and summary to be made available to outside organisations.

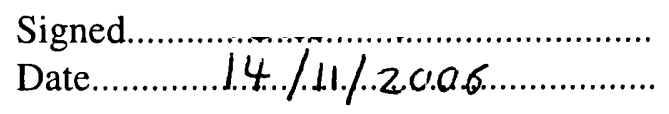




\section{Acknowledgements}

I am greatly indebted to Prof. Brzeziński for his guidance and support during the duration of my doctoral training. I would like to thank the Department of Mathematics of the University Wales Swansea for providing a happy and friendly environment for my training to take place in. Finally, I am thankful for the EPSRC support which has made all this financially viable. 


\section{Contents}

Acknowledgements iii

Preface vii

1 Introduction 1

1.1 Generalities . . . . . . . . . . . . . . . . . . . 1

1.2 Entwining structures $\ldots \ldots \ldots \ldots$

1.2.1 Origins in quantum group bundles . . . . . . . . . . . 4

1.2 .2 Conventions .................... 6

1.2 .3 Unification of modules . . . . . . . . . . . . . . 7

1.2.4 Coalgebra extensions and algebra coextensions of Galois type . . 8

1.3 Quasi-finite comodules . . . . . . . . . . . . . . . . 9

2 Corings and weak entwining structures 17

2.1 Corings ........................ . . . 17

2.1 .1 Introduction . . . . . . . . . . . . . . 17

2.1.2 Galois corings and comodules . . . . . . . . . . . 19

2.2 Weak entwining structures . . . . . . . . . . . . . 25

2.2 .1 Motivation .................... 25

2.2.2 Generalizing results from non-weak case . . . . . . . . . 28

2.3 Invertible weak entwining structures . . . . . . . . . . . . 31

3 Galois theory of $C$-rings 39

3.1 Introduction . . . . . . . . . . . . . . . . 39

3.2 Matrix rings contexts . . . . . . . . . . . . . . . . 40

3.2.1 Quasi-finite matrix ring contexts . . . . . . . . . . . . 40 40

3.2.2 Infinite (firm) matrix contexts. . . . . . . . . . . . 45

$3.3 \mathscr{A}$-coendomorphism coalgebra and Galois modules . . . . . . . . . . . 48

3.3.1 The $\mathscr{A}$-coendomorphism coalgebra and $C$-ring . . . . . . . . 48

3.3.2 Galois and principal modules . . . . . . . . . . . . . 52

3.3 .3 A Galois connection . . . . . . . . . . . . . . . . . 59

$3.4 C$-rings and invertible weak entwining structures . . . . . . . . . 62

4 Structure theorems $\quad 81$

4.1 Coextensions of self-injective algebras . . . . . . . . . . . . . 81

4.2 Extensions of self-projective coalgebras . . . . . . . . . . . 88 


\section{Preface}

The connection between geometric spaces and commutative algebras is very intimate. In algebraic geometry, geometric problems are transferred into algebraic ones by considering the functions on a space. Moving a geometric space into the realm of algebra in this way (here by geometric space we mean a locally compact Hausdorff topological space) results in a special type of algebra termed a commutative $C^{*}$-algebra. From such an algebra, up to some isomorphisms, it has been shown how a classical space can be reconstructed, via what is called the Gelfand-transform. Thus establishing a one-to-one correspondence between isomorphism classes of commutative $C^{*}$-algebras and homeomorphism classes of geometric spaces. Much of the theory of geometric spaces has been shown to have an analogue in the theory of commutative $C^{*}$-algebras. Moreover, much of this dual theory has been shown to extend to non-commutative $C^{*}$-algebras. In view of the one-to-one correspondence we have already mentioned, these non-commutative $C^{*}$-algebras can be readily thought of as a generalization of geometric space. Proceeding along these lines, the theory non-commutative geometry envisaged by Connes [19] has been highly successful, and many of the tools developed for this theory have been found to be useful when transferred back into the classical setting. One aspect of this theory which has been of interest to mathematical physicists is what happens to principal bundles in the move to this algebraic setting. Proceeding by concentrating on algebraic structure and removing some of the finer detail, a notion of what is termed a quantum principal bundle has been developed, that has the algebraic properties required of a sensible dual to a classical principal bundle. Remarkably, this notion coincided with an object, termed a Hopf-Galois extension, that had already been studied under the auspices of the theory of Hopf algebras (see [18] and [29]).

In this thesis we investigate further generalizations which can be made from the starting point of Hopf-Galois extensions. From a mathematical physics point of view, such generalizations have been required to accommodate certain classes of examples. In the development of these more general objects, new mathematical structures called entwining structures were discovered by Brzeziński and Majid [10]. In order to analyze these objects, it has proved fruitful to associate what are called corings to these objects. The theory of corings also predates non-commutative geometry, and was initiated by Sweedler in [37]. The main subject of this thesis is to develop the theory which is dual to that of corings, and then use this to provide structure theorems for what are called weak coalgebra-Galois extensions. We proceed as follows

Chapter 1 Quantum principal bundles or Hopf-Galois extensions are introduced. Entwining structures are then defined, and using this definition generalizations of quantum principal bundles are described. The category of modules for an entwining structure is 
defined and shown to be a generalizations of certain module categories appearing in the theory of Hopf algebras. $C$-Galois extensions and $A$-Galois coextension are introduced, and it is shown how to recover entwining structures from these objects. Finally an outline of the theory of quasi-finite comodules is given.

Chapter 2 An outline of the theory of $A$-corings and their comodules is described, including Galois theory. It is shown how to associate an $A$-coring to an entwining structure. Weak entwining structures and weak $C$-Galois extensions are then introduced, and it is shown how to associate a weak entwining structure to a weak $C$-Galois extension. Following joint work with T. Brzeziński and A. P. Wrightson [13], a notion of invertibility for weak entwining structures is introduced, and the consequences of the definition for associated $A$-corings and modules are explored.

Chapter 3 Following joint work with T. Brzeziński [12], an outline of the theory of $C$ rings and their modules is described, and the associated Galois theory developed. It is then shown how some of this theory extends to certain firm comodules of firm coalgebras. A Galois connection for matrix $C$-rings is given. It is shown how a $C$-ring can be associated to a weak entwining structure and necessary and sufficient conditions for a $C$-ring to be of entwined type are given. The implications of invertibility of a weak entwining structure for associated $C$-rings and modules are explored.

Chapter 4 Brings together structure theorems from [12] and [13]. Using the techniques developed in proceeding chapters, these structure theorems for $A$-Galois coextensions and $C$-Galois coextensions are proved. It is shown that some existing theorems can be seen as corollaries of these structure theorems. 


\section{Chapter 1}

\section{Introduction}

In this chapter, after agreeing on notation and terminology, we introduce the abstract concepts that will be expanded upon within this thesis.

\subsection{Generalities}

In this section we describe the basic notation and terminology that will be be used throughout this thesis. In what follows $k$ will be a commutative ring, although within some other sections of this thesis it will be a field.

Tensor Products The unadorned tensor product $\otimes$ will denote the tenor product over the base ring $k$. When the tensor product is over an algebra $A$ it will be denoted $\otimes$.

Identity map For any $k$-module $M, M: M \rightarrow M$ will denote the identity map.

Algebras Algebras will always be associative and unital. For a $k$-algebra $A$ we denote the

- product $\mu_{A}: A \otimes A \rightarrow A, \quad a \otimes a^{\prime} \mapsto a a^{\prime}$,

- unit $1_{A}: k \rightarrow A, \quad k \mapsto k 1_{A}$.

We simply write $\mu$ or 1 in cases where the algebra is clear from the context. We define the opposite algebra of $A$, written $A^{o p}$, to be the same as $A$ as a $k$-module but with the product

$$
\mu_{A^{o p}}: A^{o p} \otimes A^{o p} \rightarrow A^{o p}, \quad a \otimes a^{\prime} \mapsto \mu_{A}\left(a^{\prime} \otimes a\right) .
$$

Note that $1_{A}$ is a unit in $A^{o p}$.

Coalgebras Coalgebras will always be coassociative but not always counital, non counital coalgebras will be identified as such. For a (counital) $k$-coalgebra $C$ we adopt the Sweedler-Heyneman notation for the coproduct, and denote the

- coproduct $\Delta_{C}: C \rightarrow C \otimes C, \quad c \mapsto \sum c_{(1)} \otimes c_{(2)}$, 
- counit $\varepsilon_{C}: C \rightarrow k$.

We simply write $\Delta$ or $\varepsilon$ in cases where the coalgebra is clear from the context. Since the coproduct $\Delta$ will always be coassociative, for all $c \in C$, we write

$$
\Delta \circ(\Delta \otimes C)(c)=\Delta \circ(C \otimes \Delta)(c)=\sum c_{(1)} \otimes c_{(2)} \otimes c_{(3)},
$$

and follow the same pattern in cases where $\Delta$ is combined with itself more times.

Modules Let $A$ be an algebra. Then for any right $A$-module module $M$ and left $A$-module $N$ we usually denote the

- right $A$-action on $M$ by $\rho_{M}: M \otimes A \rightarrow M, \quad m \otimes a \mapsto m a$,

- left $A$-action on $N$ by ${ }_{N} \rho: A \otimes N \rightarrow N, \quad a \otimes n \mapsto a n$.

Occasionally, when more clarity is useful we denote the

- right $A$-action on $M$ by $\rho_{M}: M \otimes A \rightarrow M, \quad m \otimes a \mapsto m \cdot a$,

- left $A$-action on $N$ by ${ }_{N} \rho: A \otimes N \rightarrow N, \quad a \otimes n \mapsto a \cdot n$.

Comodules Let $C$ be a coalgebra, that is not necessarily counital. Then for any right $C$-comodule $M$ and left $C$-module $N$, we adopt the Sweedler-Heyneman notation for the coactions, and denote

- right $C$-coaction on $M$ by $\rho^{M}: M \rightarrow M \otimes C, \quad m \mapsto \sum m_{[0]} \otimes m_{[1]}$,

- left $C$-coaction on $N$ by ${ }^{N} \rho: N \rightarrow C \otimes N, \quad n \mapsto \sum n_{[-1]} \otimes n_{[0]}$.

Since the coaction $\rho^{M}$ will always be coassociative, for all $m \in M$, we write

$$
(M \otimes \Delta) \circ \rho^{M}(m)=\left(\rho^{M} \otimes C\right) \circ \rho^{M}(m)=\sum m_{[0]} \otimes m_{[1]} \otimes m_{[2]}
$$

and follow the same pattern in cases where $\Delta$ and $\rho^{M}$ are combined with each other more times. We adopt the symmetric convention for left $C$-comodules.

Cotensor product For any right $C$-module $M$ and left $C$-comodule $N$. The cotensor product is defined as the equalizer

$$
M \underset{C}{\square} N \longrightarrow M \otimes N \underset{M \otimes \rho^{N}}{\stackrel{\rho^{M} \otimes N}{\longrightarrow}} M \otimes C \otimes N
$$


Categories of modules and comodules For any $k$-algebra $A$ and $k$-coalgebra $C$ (not necessarily counital), we write

- $\mathbf{M}_{k}$ or Vect Vhen $_{k}$ is a field), for the category with objects that are $k$-modules and morphisms $\operatorname{Hom}_{k}(-,-)$ which are $k$-linear maps.

- $\mathbf{M}_{A}$, for the category with objects that are right $A$-modules and morphisms Hom $_{-A}(-,-)$ which are right $A$-linear maps.

- $\mathbf{M}^{C}$, for the category with objects that are right $C$-comodules and morphisms $\operatorname{Hom}^{-C}(-,-)$ which are right $C$-colinear maps. In cases where $C$ is not counital, right $C$-comodules do not necessarily satisfy any counitality condition.

- ${ }_{A} \mathbf{M}^{C}$, for the category with objects that are both right $C$-comodules and left $A$ modules, for which the left $A$-action is a right $C$-colinear map, or equivalently the right $C$-coaction is left $A$-linear. In either case we require that

$$
\rho^{M} \circ{ }_{M} \rho=\left({ }_{M} \rho \otimes C\right) \circ\left(A \otimes \rho^{M}\right) .
$$

The morphisms in this category will be right $C$-colinear left $A$-linear maps.

Symmetrically, we define ${ }_{A} \mathbf{M},{ }^{C} \mathbf{M}$ and ${ }^{C} \mathbf{M}_{A}$, in the obvious way.

Dual modules For any $M \in \mathbf{M}_{A}$ we denote the dual left $A$-module $\operatorname{Hom}_{-A}(M, A)$ by $M^{*}$. Similarly for any $N \in{ }_{A} \mathbf{M}$ we denote the dual right $A$-module $\operatorname{Hom}_{A-}(N, A)$ by ${ }^{*} N$.

Notions of projectivity Let $A$ be a $k$-algebra and $C$ be a $k$-coalgebra then we say that

- $M \in \mathbf{M}^{C}$ is a projective $C$-comodule provided that, for any surjective map of right $C$-comodules $\pi: M^{\prime} \rightarrow M^{\prime \prime}$ and any right $C$-comodule map $f: M \rightarrow M^{\prime \prime}$, there exists a right $C$-comodule map $g: M \rightarrow M^{\prime}$ such that $\pi \circ g=f$. Or equivalently, in the language of category theory, for any epimorphism $\pi: M^{\prime} \rightarrow M^{\prime \prime}$ in $\mathbf{M}^{C}$, the mapping of sets

$$
\operatorname{Hom}^{-C}(M, \pi): \operatorname{Hom}^{-C}\left(M, M^{\prime}\right) \rightarrow \operatorname{Hom}^{-C}\left(M, M^{\prime \prime}\right), \quad f \mapsto \pi \circ f
$$

is surjective.

- $M \in{ }^{C} \mathbf{M}_{A}$ is a $C$-equivariantly projective right $A$-module provided that, for any epimorphism $\pi: M^{\prime} \rightarrow M^{\prime \prime}$ in ${ }^{C} \mathbf{M}_{A}$, which splits in ${ }^{C} \mathbf{M}$, and morphism $f: M \rightarrow M^{\prime \prime}$ in ${ }^{C} \mathbf{M}_{A}$, there exists a morphism $g: M \rightarrow M^{\prime \prime}$ in ${ }^{C} \mathbf{M}_{A}$, such that $\pi \circ g=f$. Or equivalently, the map $\rho_{M}: M \otimes A \rightarrow M$ is a split epimorphism in ${ }^{C} \mathbf{M}_{A}$.

- $M \in \mathbf{M}_{A}$ is a $k$-relatively projective right $A$-module provided that, for any epimorphism $\pi: M^{\prime} \rightarrow M^{\prime \prime}$ in $\mathbf{M}_{A}$, which splits in $\mathbf{M}_{k}$, and morphism $f: M \rightarrow M^{\prime \prime}$, there exists a morphism $g: M \rightarrow M^{\prime}$ in $\mathbf{M}_{A}$ such that $\pi \circ g=f$. Or equivalently, any epimorphism $\pi: N \rightarrow M$ in $\mathbf{M}_{A}$, which splits in $\mathbf{M}_{k}$, splits in $\mathbf{M}_{A}$.

Note that the list of equivalent conditions given above is not exhaustive. Similarly we define left handed versions of these notions. 
Notions of injectivity The notions of injectivity we shall use, are those which are dual to those we have explained for projectivity.

The convolution product Let $C$ be a $k$-coalgebra and $A$ be a $k$-algebra. Then it possible to define a product on $\operatorname{Hom}_{k}(C, A)$ by defining, for all $f, g \in \operatorname{Hom}_{k}(C, A)$ and $c \in C$,

$$
(f * g)(c)=f\left(c_{(1)}\right) g\left(c_{(2)}\right) .
$$

This product is termed the convolution product. Note that with this product $\operatorname{Hom}_{k}(C, A)$ is an algebra, termed the convolution algebra, with unit element $1_{A} \circ \varepsilon_{C}$.

Bialgebras and Hopf algebras Let $H$ be a $k$-module that is both a $k$-algebra and a $k$ coalgebra. Then we shall say that $H$ is a $k$-bialgebra provided the $\mu_{H}$ and $1_{H}$, are comultiplicative, and $\Delta_{H}$ and $\varepsilon_{H}$ are multiplicative. If moreover, the identity map $H$ has an inverse $S$ in the convolution algebra $\operatorname{Hom}_{k}(H, H)$, then we shall say that $H$ is a Hopf algebra with antipode $S$.

Comodule algebras and module coalgebras Let $A$ be a $k$-algebra, $B$ be a $k$-bialgebra and $C$ be a $k$-coalgebra. Then we shall say that $A$ is a right $B$-comodule algebra provided, $A \in \mathbf{M}^{B}$ and the coaction is a multiplicative map, where $A \otimes B$ has the tensor product algebra structure. Similarly we shall say that $C$ is a right $B$-module coalgebra provided, $C \in \mathbf{M}_{B}$ and the action is a comultiplicative map, where $C \otimes B$ has the tensor product coalgebra structure. Similarly we can define left landed versions.

\subsection{Entwining structures}

\subsubsection{Origins in quantum group bundles}

Entwining structures where first introduced in [10] in order to broaden the following definition:

Definition 1.2.1. $P=P(B, H)$ is a quantum principal bundle with universal differential calculus, structure quantum group $H$ and base $B$ or a Hopf-Galois extension if and only if:

1. $H$ is a Hopf algebra.

2. $P$ is a right $H$-comodule algebra.

3. $B=P^{\mathrm{coH}}:=\left\{u \in P \mid \rho^{P}(u)=u \otimes 1\right\}$.

4. $\operatorname{can}_{P}: P \otimes_{B} P \rightarrow P \otimes H, \quad \operatorname{can}_{P}=(\mu \otimes H) \circ\left(P \otimes \rho^{P}\right)$ is a bijection.

This notion of a Hopf-Galois extension, in this form, was introduced in [29], and built upon an earlier definition given in [18]. This object can be thought of as the object dual to a principal bundle as defined in ordinary differential geometry (cf. [34]). The differential geometric meaning of a Hopf-Galois extension was further explored in [11], where the theory of connections was developed. Importantly from this standpoint it can be shown 
that the universal $n$-forms on $\mathrm{P}$, defined as $\Omega^{n} P:=\left\{\omega \in P^{\otimes n+1}: \quad \forall i \in 1, \ldots, n, \quad \mu_{i} \omega=\right.$ $0\}$ where $\mu_{i}$ denotes the multiplication in $P$ acting on the $i$ and $i+1$ factors in $P^{\otimes n+1}$, admit a right $H$-coaction. Not to loose this geometric meaning it was critical that any useful generalization should also have this property. Also, in order to include specific examples it was desirable that the conditions on the structure quantum group $H$ should be relaxed in order to accommodate generic coalgebras. With these considerations in mind a generalization of a quantum principal bundle with universal differential calculus was proposed as follows.

Definition 1.2.2. A coalgebra $C$ and an algebra $P$ are entwined if there exists a map $\psi$ : $C \otimes P \rightarrow P \otimes C$ such that the following diagram commutes:

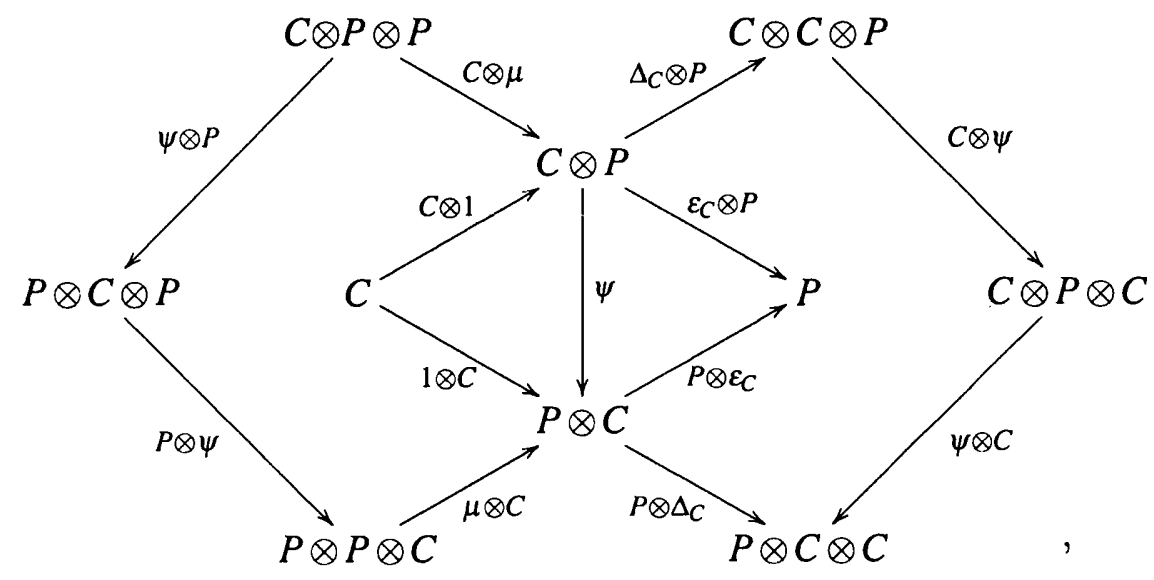

The map $\psi$ is referred to as the entwining map.

Let $n$ be a positive integer, for $1 \leq i \leq n$ let $\psi_{i, i+1}$ symbolize the entwining map applied to the $i$ and $i+1$ legs of $P^{\otimes i-1} \otimes C \otimes P \otimes P^{\otimes n-i-1}$ then the following Proposition, taken from [10], defines a generalization of a quantum principal bundle.

Proposition 1.2.3. Let $C, P$ be entwined by $\psi$. For every group-like element $e \in C$ we have the following:

1. For any positive $n, P^{\otimes n}$ is a right $C$-comodule with the coaction $\rho^{P^{\otimes n}}=\psi_{n, n+1} \circ$ $\psi_{n-1, n} \circ \ldots \circ \psi_{1,2} \circ\left(\bar{\eta}_{C} \otimes P^{\otimes n}\right)$, where $\bar{\eta}_{C}: k \rightarrow C, \alpha \mapsto \alpha e$.

2. The coaction $\rho^{p^{\otimes n}}$ restricts to a coaction on $\Omega^{n} P$.

3. $M=P_{e}^{\mathrm{coC}}=\left\{u \in p: \rho^{P}(u)=u \otimes e\right\}$ is a subalgebra of $P$.

4. The k-linear map $\operatorname{can}_{P}: P \underset{B}{\otimes} P \rightarrow P \otimes C, \quad u \otimes_{B} v \mapsto u \psi(e \otimes v)$ is well-defined. If can can is a bijection we say that we have a $\psi$-principal bundle $P(M, C, \psi, e)$.

Proof. See [10, Proposition 2.2].

In the following example, again taken from [10], we see that this notion is indeed a generalization of a quantum principal bundle. 
Example 1.2.4. Let $H$ be a Hopf algebra and $P$ be a right $H$-comodule algebra. The $k$-linear map $\psi: H \otimes P \rightarrow P \otimes H$ defined by $\psi(h \otimes p)=\sum p_{[0]} \otimes h p_{[1]}$ entwines $H$ and $P$. Therefore with this choice of $\psi, P(M, H)$ a quantum principal bundle with universal differential calculus can be seen as a $\psi$-principal bundle $P\left(M, H, \psi, 1_{H}\right)$.

Proof. See [10, Example 2.3].

Remark 1.2.5. Just as for Hopf algebras the diagram which must be satisfied for an entwined algebra and coalgebra is formally self-dual, in that by swapping $\mu_{P}$ with $\Delta_{C}, 1_{P}$ with $\varepsilon_{C}$ and $P$ with $C$, you arrive back at the initial diagram. This allows proposition 1.2.3 to be dualized as follows.

Proposition 1.2.6. Let $C, P$ be entwined by $\psi: C \otimes P \rightarrow P \otimes C$. For every algebra character $\kappa: P \rightarrow k$ we have the following:

1. For any positive integer $n, C^{\otimes n}$ is a right $P$-module with the action $\rho_{C^{\otimes n}}:=(\kappa \otimes$ $\left.P^{\otimes n}\right) \circ \psi_{1,2} \circ \psi_{2,3} \circ \ldots \circ \psi_{n, n+1}$.

2. The action $\rho_{C}^{\otimes n}$ maps $\Delta_{C}^{n}(C)$ to itself.

3. The subspace $I_{k}=\operatorname{span}\{c u-c \kappa(u) \mid c \in C, u \in P\}$ is a coideal. Hence $D=C / I_{\kappa}$ is a coalgebra. Denote the corresponding canonical surjection by $\pi_{\kappa}: C \rightarrow D$.

4. There is a map $\beta: C \otimes P \rightarrow C \square C$ defined by $\beta(c \otimes u)=\sum c_{(1)} \otimes c_{(2)} u$, where $C$ is viewed as a $(D, D)$-bicomodule via the map $\pi_{\kappa}: C \rightarrow D$. If $\beta$ is a bijection we say that $C(D, P, \psi, \kappa)$ is a dual $\psi$-principal bundle.

Proof. See [10, Proposition 2.6].

\subsubsection{Conventions}

Beyond the original situation for which they were intended, an entwined pair of a coalgebra and an algebra was found to have a lot of algebraic structure. As such, these objects have been studied in their own right. We now consider triples $(A, C, \psi)$ consisting of an algebra A, coalgebra $\mathrm{C}$ and some map $\psi$. Depending on the properties of $\psi$ we make the following definitions.

Definition 1.2.7. If $C$ and $A$ are entwined with entwining map $\psi$ as in Definition 1.2.2 (with $P=A$ ) we say that $(A, C, \psi)$ is a right-right entwining structure. Writing, for all $a \in A$ and $c \in C, \psi(c \otimes a)=\sum_{\alpha} a_{\alpha} \otimes c^{\alpha}$ this means that

$$
\begin{array}{r}
\sum_{\alpha}(a b)_{\alpha} \otimes c^{\alpha}=\sum_{\alpha, \beta} a_{\alpha} b_{\beta} \otimes c^{\alpha \beta}, \\
\sum_{\alpha} a_{\alpha} \varepsilon_{C}\left(c^{\alpha}\right)=\sum_{\alpha} \varepsilon_{C}(c) a, \\
\sum_{\alpha} a_{\alpha} \otimes \Delta_{C}\left(c^{\alpha}\right)=\sum_{\alpha, \beta} a_{\alpha \beta} \otimes c_{(1)}^{\beta} \otimes c_{(2)}{ }^{\alpha}, \\
\sum_{\alpha} 1_{\alpha} \otimes c^{\alpha}=1 \otimes c .
\end{array}
$$


The category of right-right entwining structures $\mathbf{E}_{\bullet}^{\bullet}$ is defined to have objects which are entwining structures and morphisms which are pairs $(f, g):(A, C, \psi) \rightarrow\left(A^{\prime}, C^{\prime}, \psi^{\prime}\right)$ consisting of an algebra map $f: A \rightarrow A^{\prime}$ and a coalgebra map $g: C \rightarrow C^{\prime}$ such that

$$
(f \otimes g) \circ \psi=\psi^{\prime} \circ(g \otimes f) .
$$

Definition 1.2.8. If $C$ and $A$ are entwined with entwining map $\psi: A \otimes C \rightarrow C \otimes A$ as in Definition 1.2 .2 but with $\psi$-arrows reversed we say that $(A, C, \psi)$ is a left-left entwining structure. Writing, for all $a \in A$ and $c \in C, \psi(a \otimes c)=\sum_{E} c_{E} \otimes a^{E}$ this means that

$$
\begin{array}{r}
\sum_{E} c_{E} \otimes(a b)^{E}=\sum_{E, F} c_{E F} \otimes a^{F} b^{E}, \\
\sum_{E} \varepsilon_{C}\left(c_{E}\right) a^{E}=\sum_{E} a \varepsilon_{C}(c), \\
\sum_{E} \Delta_{C}\left(c_{E}\right) \otimes a^{E}=\sum_{E, F} c_{(1) E} \otimes c_{(2) F} \otimes a^{E F}, \\
\sum_{E} c_{E} \otimes 1^{E}=c \otimes 1 .
\end{array}
$$

The category of left-left entwining structures is denoted by $: \mathbf{E}$.

Interestingly if $(A, C, \psi)$ is a right-right entwining structure with invertible $\psi$ then $\left(A, C, \psi^{-1}\right)$ is a left-left entwining structure. In the case when $\psi$ is invertible the triple $(A, C, \psi)$ is called an invertible entwining structure. Similarly one can define the notions of left-right and right-left entwining structures, for which the corresponding categories are denoted. $\mathbf{E}^{\bullet}$ and ${ }^{\bullet} \mathbf{E}$. respectively. We refer to [17] for more details about all possible conventions and definitions of categories of entwining structures.

\subsubsection{Unification of modules}

In the study of Hopf algebras many different categories of associated modules have appeared. These include Hopf modules [36], relative Hopf modules [21] [39] and YetterDrinfeld modules [42] [31]. In order to obtain a better understanding of these modules it has proved profitable to bring the theory of these modules together by considering them each as part of a more general category of modules. Such a unifying category of modules was proposed, independently in papers by Doi [22] and Koppinen [28], as follows.

Definition 1.2.9. A right-right Doi-Koppinen structure is a triple $(H, A, C)$ where $H$ is bialgebra, $A$ a right $H$-comodule algebra and $C$ a right $H$-module coalgebra. The category of modules corresponding to such a triple, denoted $\mathbf{M}(H)_{A}^{C}$, has objects which are right $C$ comodules with a right $A$-action satisfying the compatibility condition, that for all $m \in M$ and $a \in A$,

$$
\rho^{M}(m a)=\sum m_{[0]} a_{[0]} \otimes m_{[1]} a_{[1]}
$$

where $\rho^{M}(m)=\sum m_{[0]} \otimes m_{[1]} \in M \otimes H$ and $\rho^{A}(a)=\sum a_{[0]} \otimes a_{[1]} \in A \otimes H$, and morphism which are right $A$-linear and right $C$-colinear maps. Objects of this category are called unifying or Doi-Koppinen Hopf-modules. 
Similarly one can define left-right, right-left and left-left Doi-Koppinen structures and their corresponding categories of modules, denoted ${ }_{A} \mathbf{M}(H)^{C},{ }^{C} \mathbf{M}(H)_{A}$ and ${ }_{A}^{C} \mathbf{M}(H)$ respectively.

Remark 1.2.10. For any right-right Doi-Koppinen structure $(H, A, C)$ the map $\psi: C \otimes A \rightarrow$ $A \otimes C$ defined $\psi_{H}(c \otimes a)=\sum a_{[0]} \otimes c a_{[1]}$ gives a corresponding right-right entwining structure $\left(A, C, \psi_{H}\right)$. In this way one sees that right-right entwining structures are at least as general as right-right Doi-Koppinen structures. Under the assumption that $A$ is finitely generated and projective as a $k$-module it has been shown in [41] that every right-right entwining structure can be generated in this way. However for general algebras and coalgebras this is not true and an example of an entwining structure not arising from a DoiKoppinen structure was provided in [32]. Hence in some sense entwining structures can be thought of as a generalization of bialgebras.

Just as a category of modules can be associated to a Doi-Koppinen structure, one can associate a category of modules to an entwining structure, as described in [7], as follows.

Definition 1.2.11. For a right-right entwining structure $(A, C, \psi)$ the category of rightright entwined modules, denoted $\mathbf{M}(\psi)_{A}^{C}$, has objects $M$ which are right $C$-comodules with a right $A$-action satisfying the compatibility condition, for all $m \in M$ and $a \in A$

$$
\rho^{M}(m a)=\sum_{\alpha} m_{[0]} a_{\alpha} \otimes m_{[1]}^{\alpha},
$$

and morphisms which are right $A$-linear and right $C$-colinear maps.

Similarly for left-right, right-left and left-left entwining structures one can define their corresponding categories of entwined modules, denoted ${ }_{A} \mathbf{M}(\psi)^{C},{ }^{C} \mathbf{M}(\psi)_{A}$ and ${ }_{A}^{C} \mathbf{M}(\psi)$ respectively. In the notation of Remark 1.2 .10 it is easy to see that $\mathbf{M}(H)_{A}^{C}=\mathbf{M}\left(\psi_{H}\right)_{A}^{C}$ hence entwined modules are a generalization of unifying Hopf-modules. Many results about unifying Hopf-modules generalize into this broader setting.

\subsubsection{Coalgebra extensions and algebra coextensions of Galois type}

Just as a quantum principle bundle with universal differential calculus $P(B, H)$ can be described as an Hopf-Galois extension, in this section we mention how a principal $\psi$-bundle and dual principal $\psi$-bundle, can equally be viewed as certain types of algebra extension and coalgebra coextension respectively. In particular following [9] we mention how the entwining map can be recovered from these (co)extensions.

Definition 1.2.12. Let $C$ be a coalgebra and $A$ be an algebra that is also a right $C$-comodule. Then

$$
B:=A^{\mathrm{coC}}:=\left\{b \in A \mid \rho^{A}(b a)=b \rho^{A}(a), \forall a \in A\right\},
$$

is a subalgebra of $A$, which is termed the subalgebra of (right) coinvariants. We say that $B \subseteq A$ is a (right) coalgebra-Galois extension (or $C$-Galois extension) provided that the left $A$-linear right $C$-colinear map

$$
\operatorname{can}_{A}: A \underset{B}{\otimes} A \rightarrow A \otimes C, \quad \operatorname{can}_{A}:=(\mu \otimes C) \circ\left(A \underset{B}{\otimes} \rho^{A}\right),
$$

is bijective. 
Now for such an extension there is the following result.

Theorem 1.2.13. Let $B \subseteq A$ be a $C$-Galois extension. Then there exists a unique map $\psi: C \otimes A \rightarrow A \otimes C$ entwining $C$ with $A$ and such that $A \in \mathbf{M}(\psi)_{A}^{C}$ with the structure maps $\mu$ and $\rho^{A}$. This entwining map is referred to as the canonical entwining map associated to the $C$-Galois extension $B \subseteq A$.

Proof. For a $C$-Galois extension define

$$
\psi: C \otimes A \rightarrow A \otimes C, \quad \psi(c \otimes a)=\operatorname{can}_{A}\left(\left(\operatorname{can}_{A}^{-1}(1 \otimes c)\right) a\right),
$$

see [9, Theorem 2.7] for detailed proof.

Dually there is the following.

Definition 1.2.14. Let $A$ be an algebra and $C$ be a coalgebra that is also a right $A$-module. Then the space

$$
I:=\operatorname{span}\left\{\sum(c a)_{(1)} \alpha\left((c a)_{(2)}\right)-\sum c_{(1)} \alpha\left(c_{(2)} a\right) \mid a \in A, c \in C, \alpha \in \operatorname{Hom}_{k}(C, k)\right\}
$$

is a coideal of $C$ (see [9, Lemma 3.2] for a proof of this). Now $B:=C / I$ is a coalgebra and we say $C \rightarrow B$ is an (right) algebra-Galois coextension (or an A-Galois coextension) provided that the left $A$-linear right $C$-colinear map

$$
\beta: C \otimes A \rightarrow C \square_{B} C, \quad \beta:=\left(C \otimes \rho_{C}\right) \circ\left(\Delta_{C} \otimes A\right)
$$

is bijective.

In the algebra-Galois coextension case there is a dual result.

Theorem 1.2.15. Let $C \rightarrow B$ be an $A$-Galois coextension. Then there exists a unique map $\psi: C \otimes A \rightarrow A \otimes C$ entwining $C$ with $A$ and such that $C \in \mathbf{M}(\psi)_{A}^{C}$ with the structure maps $\Delta_{C}$ and $\rho_{C}$. This entwining map is referred to as the canonical entwining map associated to the $A$-Galois coextension $C \rightarrow B$.

Proof. For an $A$-Galois coextension define

$$
\psi: C \otimes A \rightarrow A \otimes C, \quad \psi(c \otimes a)=\sum\left(\left(\varepsilon_{C} \otimes A\right) \circ \beta^{-1}\left(c_{(1)} \otimes\left(c_{(2)} a\right)_{(1)}\right)\right) \otimes\left(c_{(2)} a\right)_{(2)}
$$

see $[9$, Theorem 3.5] for detailed proof.

\subsection{Dual notions of finitely-generated and projective for comodules}

In this section we recall the dual notions of finitely-generated and projective for comodules. Following [14, Section 12] and [38] we also review further constructions which can be made from comodules satisfying such notions, in particular those which will be used in subsequent chapters. Throughout this section we work over a field $k$. 
Definition 1.3.1. A right $C$-comodule $M$ is said to be quasi-finite, provided that the functor $-\otimes M:$ Vect $_{k} \rightarrow \mathbf{M}^{C}$ has a left adjoint. If such a functor exists it is denoted by $h_{C}(M,-)$ : $\mathbf{M}^{C} \rightarrow$ Vect $_{k}$ and called the co-hom functor.

Example 1.3.2. Since $\operatorname{Hom}^{-C}(M, V \otimes C) \simeq \operatorname{Hom}_{k}(M, V)$ it is clear that the forgetful functor $\mathbf{M}^{C} \rightarrow$ Vect $_{k}$ is the left adjoint of $-\otimes C:$ Vect $_{k} \rightarrow \mathbf{M}^{C}$, so $C$ is quasi-finite as a right $C$-comodule.

Remark 1.3.3. Suppose that $\mathrm{M}$ is a quasi-finite right $C$-comodule, $N$ is a right $C$-comodule and $V$ is a vector space over $k$. Let

$$
\Omega_{N, V}: \operatorname{Hom}_{k}\left(h_{C}(M, N), V\right) \rightarrow \operatorname{Hom}^{-C}(N, V \otimes M)
$$

denote the functorial isomorphism required by Definition 1.3.1. Given any map $f \in \operatorname{Hom}^{-C}(N, V \otimes M)$ let $u \in \operatorname{Hom}_{k}\left(h_{C}(M, N), V\right)$ be the unique map such that $\Omega_{N, V}(u)=$ $f$. Now recall that $\Omega_{N, V}$ can be expressed in terms of the corresponding unit of adjunction, $\varphi_{N}: N \rightarrow h_{C}(M, N) \otimes M$, in this case as $\Omega_{N, V}(u)=(u \otimes M) \circ \varphi_{N}$. Hence it is clear that any right $C$-colinear map $f: N \rightarrow V \otimes M$ can be factorized as a composition

$$
N \stackrel{\varphi_{N}}{\longrightarrow} h_{C}(M, N) \otimes M \stackrel{u \otimes M}{\longrightarrow} V \otimes M
$$

for a unique choice of $k$-linear map $u: h_{C}(M, N) \rightarrow V$.

Remark 1.3.4. By the previous remark any right $C$-comodule map $f: N \rightarrow L$ induces a map $h_{C}(M, f): h_{C}(M, N) \rightarrow h_{C}(M, L)$ determined uniquely by the condition

$$
\varphi_{L} \circ f=\left(h_{C}(M, f) \otimes M\right) \circ \varphi_{N} .
$$

Since the result of applying the co-hom functor to a map $f: N \rightarrow L$ produces a map satisfying this condition, this notation is justified, i.e. the result of applying the co-hom functor to a map $f$ results in the map $h_{C}(M, f)$.

Proposition 1.3.5. Suppose that $M$ is a quasi-finite right $C$-comodule, $N$ is a right $C$ comodule and $V$ is a vector space over $k$. Then the map

$$
\Psi_{V, N}: h_{C}(M, V \otimes N) \rightarrow V \otimes h_{C}(M, N),
$$

determined uniquely by the condition

$$
V \otimes \varphi_{N}=\left(\Psi_{V, N} \otimes M\right) \circ \varphi_{V \otimes N}
$$

is an isomorphism.

Proof. See [40, Proposition 3.2].

Corollary 1.3.6. The maps $\Psi_{-,-}$defined above are the natural transformations for an isomorphism of functors

$$
h_{C}(M,-\otimes-) \simeq-\otimes h_{C}(M,-)
$$


Proof. To see that these are natural transformations in the first component, we need to check that, for all vector spaces $\mathrm{V}$ and $\mathrm{W}$, and $k$-linear maps $f: V \rightarrow W$, the diagram

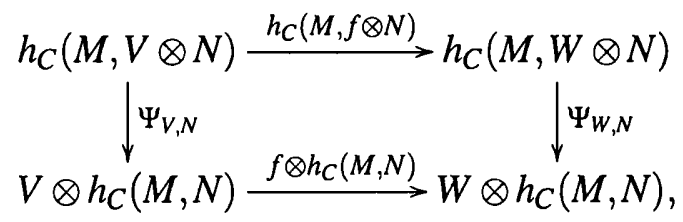

is commutative. To check this observe

$$
\begin{aligned}
\left(\left(\Psi_{W, N} \circ h_{C}(M, f \otimes N)\right) \otimes M\right) \circ \varphi_{V \otimes N} & =\left(\Psi_{W, N} \otimes M\right) \circ \varphi_{W \otimes N} \circ(f \otimes N) \\
& =\left(W \otimes \varphi_{N}\right) \circ(f \otimes N) \\
& =\left(f \otimes h_{C}(M, N) \otimes M\right) \circ\left(V \otimes \varphi_{N}\right) \\
& =\left(f \otimes h_{C}(M, N) \otimes M\right) \circ\left(\Psi_{V, N} \otimes M\right) \circ \varphi_{V \otimes N} \\
& \left.=\left(\left(f \otimes h_{C}(M, N)\right) \circ \Psi_{V, N}\right) \otimes M\right) \circ \varphi_{V \otimes N}
\end{aligned}
$$

By multiple use of the defining properties of $\Psi_{-, N}$ and $h_{C}(M, f \otimes N)$. Hence the commutativity follows by the universal mapping property observed in Remark 1.3.3. A similar calculation shows these transformations are natural in the second component.

Proposition 1.3.7. Let $M$ be a quasi-finite right $C$-comodule. Suppose that $N$ is a $(F, C)$ bicomodule then $h_{C}(M, N)$ is a left $F$-comodule with ${ }^{h_{C}(M, N)} \rho:=\Psi_{F, N} \circ h_{C}\left(M,{ }^{N} \rho\right)$.

Proof. To see this coaction is coassociative observe that the diagram

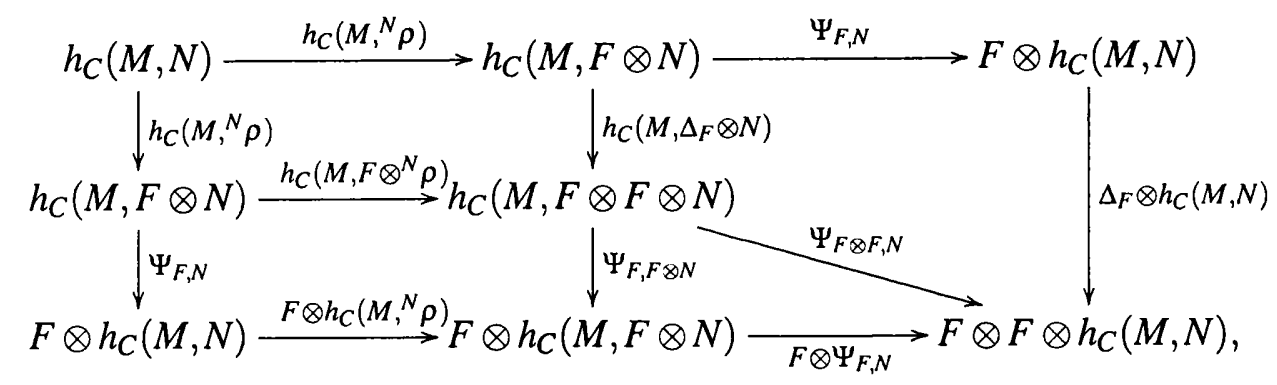

is commutative. The commutativity of the top left quadrilateral in the diagram follows from the coassociative of ${ }^{N} \rho$ and the commutativity of the other two quadrilaterals follows since $\Psi_{-,-}$is natural in either component, as shown in Corollary 1.3.6. To see that the triangle is commutative observe that, by the defining property of $\Psi_{-,-}$,

$$
\begin{aligned}
\left(\Psi_{F \otimes F, N} \otimes M\right) \varphi_{F \otimes F \otimes N} & =F \otimes F \otimes \varphi_{N} \\
& =F \otimes\left(\left(\Psi_{F, N} \otimes M\right) \circ \varphi_{F \otimes N}\right) \\
& =\left(F \otimes \Psi_{F, N} \otimes M\right) \circ\left(F \otimes \varphi_{F \otimes N}\right) \\
& =\left(F \otimes \Psi_{F, N} \otimes M\right) \circ\left(\Psi_{F, F \otimes N} \otimes M\right) \circ \varphi_{F \otimes F \otimes N} \\
& =\left(\left(\left(F \otimes \Psi_{F, N}\right) \circ \Psi_{F, F \otimes N}\right) \otimes M\right) \circ \varphi_{F \otimes F \otimes N} .
\end{aligned}
$$


Now reading around the edges of the diagram exhibits the coassociativity. By making similar observations it is possible to show that the diagram

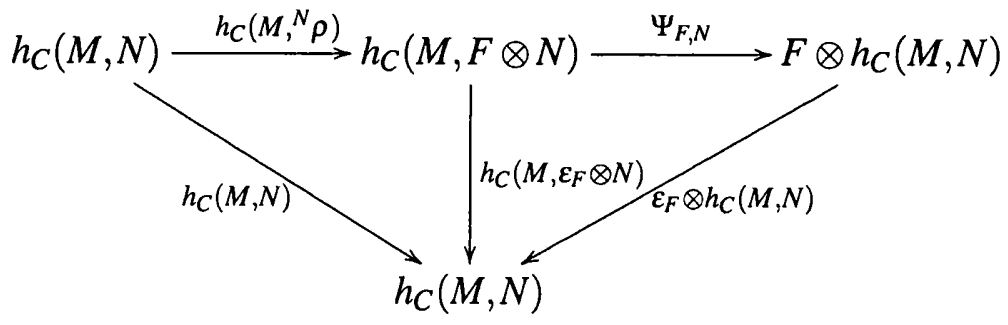

is commutative, and reading around the edges of this demonstrates that the left coaction is counital.

Proposition 1.3.8. Let $M$ be a $(D, C)$-bicomodule such that $M$ is quasi-finite as a right $C$-comodule. Suppose $N$ is a right $C$-comodule then $h_{C}(M, N)$ is a right $D$-comodule with coaction $\rho^{h_{C}(M, N)}$ uniquely determined by the condition

$$
\left(h_{C}(M, N) \otimes^{M} \rho\right) \circ \varphi_{N}=\left(\rho^{h_{C}(M, N)} \otimes M\right) \circ \varphi_{N} .
$$

Proof. The map $\rho^{h_{C}(M, N)}$ is coassociative since

$$
\begin{aligned}
\left(\left(\rho^{h_{C}(M, N)} \otimes D\right) \circ \rho^{h_{C}(M, N)} \otimes M\right) \circ \varphi_{N} & =\left(\rho^{h_{C}(M, N)} \otimes D \otimes M\right) \circ\left(\rho^{h_{C}(M, N)} \otimes M\right) \circ \varphi_{N} \\
& =\left(\rho^{h_{C}(M, N)} \otimes D \otimes M\right) \circ\left(h_{C}(M, N) \otimes{ }^{M} \rho\right) \circ \varphi_{N} \\
& =\left(h_{C}(M, N) \otimes D \otimes{ }^{M} \rho\right) \circ\left(\rho^{h_{C}(M, N)} \otimes M\right) \circ \varphi_{N} \\
& =\left(h_{C}(M, N) \otimes D \otimes{ }^{M} \rho\right) \circ\left(h_{C}(M, N) \otimes{ }^{M} \rho\right) \circ \varphi_{N} \\
& =\left(h_{C}(M, N) \otimes \Delta_{D} \otimes M\right) \circ\left(h_{C}(M, N) \otimes{ }^{M} \rho\right) \circ \varphi_{N} \\
& =\left(h_{C}(M, N) \otimes \Delta_{D} \otimes M\right) \circ\left(\rho^{h_{C}(M, N)} \otimes M\right) \circ \varphi_{N} \\
& =\left(\left(\left(h_{C}(M, N) \otimes \Delta_{D}\right) \circ \rho^{h_{C}(M, N)}\right) \otimes M\right) \circ \varphi_{N} .
\end{aligned}
$$

These equalities follow by multiple use of the defining property of $\rho^{h_{C}(M, N)}$ and the coassociativity of ${ }^{M} \rho$. Now by the adjointness it is clear that $\rho^{h_{C}(M, N)}$ is coassociative. Similarly

$$
\begin{aligned}
\left(\left(h_{C}(M, N) \otimes \varepsilon_{D}\right) \circ \rho^{h_{C}(M, N)} \otimes M\right) \circ & \varphi_{N} \\
& =\left(h_{C}(M, N) \otimes \varepsilon_{D} \otimes M\right) \circ\left(\rho^{h_{C}(M, N)} \otimes M\right) \circ \varphi_{N} \\
& =\left(h_{C}(M, N) \otimes \varepsilon_{D} \otimes M\right) \circ\left(h_{C}(M, N) \otimes{ }^{M} \rho\right) \circ \varphi_{N} \\
& =\left(h_{C}(M, N) \otimes M\right) \circ \varphi_{N},
\end{aligned}
$$

by the defining property of $\rho^{h_{C}(M, C)}$ and the counitality of ${ }^{M} \rho$. Therefore, by the adjointness it is clear the $\rho^{h_{C}(M, C)}$ is counital.

Remark 1.3.9. If $M$ is a $(D, C)$ bicomodule which is quasi-finite as a right $C$-comodule, then the previous propositions allow the co-hom functor to be viewed as a functor $h_{C}(M,-)$ : $\mathbf{M}^{C} \rightarrow \mathbf{M}^{D}$.

Proposition 1.3.10. Let $M$ be a $(D, C)$-bicomodule such that $M$ is quasi-finite as a right $C$ comodule. Suppose that $N$ is an $(F, C)$-bicomodule. Then $h_{C}(M, N)$ is an $(F, D)$-bicomodule with the coactions given in the previous propositions. 
Proof. First observe the diagram

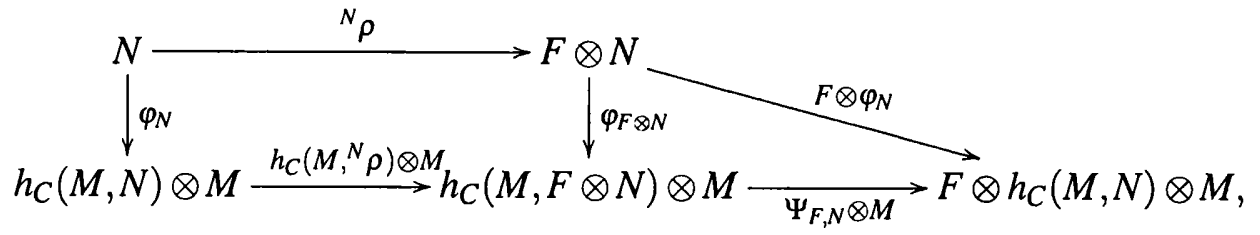

is clearly commutative and so $\varphi_{N}$ is left $F$-colinear. Now compute

$$
\begin{aligned}
\left(\left(\left({ }^{h_{C}(M, N)} \rho \otimes D\right) \circ \rho^{h_{C}(M, N)}\right) \otimes M\right) \circ \varphi_{N} \\
= \\
=\left({ }^{h_{C}(M, N)} \rho \otimes D \otimes M\right) \circ\left(\rho^{h_{C}(M, N)} \otimes M\right) \circ \varphi_{N} \\
=\left({ }^{h_{C}(M, N)} \rho \otimes D \otimes M\right) \circ\left(h_{C}(M, N) \otimes{ }^{M} \rho\right) \circ \varphi_{N} \\
=\left(F \otimes h_{C}(M, N) \otimes{ }^{M} \rho\right) \circ\left({ }^{h_{C}(M, N)} \rho \otimes M\right) \circ \varphi_{N} \\
=\left(F \otimes h_{C}(M, N) \otimes{ }^{M} \rho\right) \circ\left(F \otimes \varphi_{N}\right) \circ{ }^{N} \rho \\
=\left(F \otimes \rho^{h_{C}(M, N)} \otimes M\right) \circ\left(F \otimes \varphi_{N}\right) \circ{ }^{N} \rho \\
=\left(F \otimes \rho^{h_{C}(M, N)} \otimes M\right) \circ\left({ }^{h_{C}(M, N)} \rho \otimes M\right) \circ \varphi_{N} \\
=\left(\left(\left(F \otimes \rho^{h_{C}(M, N)}\right) \circ{ }^{h_{C}(M, N)} \rho\right) \otimes M\right) \circ \varphi_{N} .
\end{aligned}
$$

This follows by making multiple use of the defining property of the map $\rho^{h_{C}(M, N)}$ and the left $F$-colinearity of $\varphi_{N}$. Now by the universal mapping property mentioned in Remark 1.3.3 the previous computation shows that the coactions must be compatible.

Proposition 1.3.11. Suppose $M$ is quasi-finite as a right $C$-comodule. For each right $C$ comodule $N$, the map $\delta_{N}: h_{C}(M, N) \rightarrow N \square_{C} h_{C}(M, C)$ uniquely determined by the condition

$$
\left(\delta_{N} \otimes M\right) \circ \varphi_{N}=\left(N \square_{C} \varphi_{C}\right) \circ \rho^{N}
$$

has the following properties.

1. If $N$ is a $(D, C)$-bicomodule then $\delta_{N}$ is left $D$-colinear with the left $D$-coaction described in Proposition 1.3.7.

2. If $M$ is a $(D, C)$-bicomodule then $\delta_{N}$ is right $D$-colinear with the right $D$-coaction described in Proposition 1.3.8.

Proof. This is a straight forward calculation and is left to the reader.

The observation made in Remark 1.3 .9 can be further developed to give an alternative definition of quasi-finite for bicomodules as follows.

Theorem 1.3.12. Suppose that $M$ is a $(D, C)$-bicomodule, then $M$ is quasi-finite as a right $C$-comodule if and only if the functor $-\square_{D} M: \mathbf{M}^{D} \rightarrow \mathbf{M}^{C}$ has a left adjoint, moreover this left adjoint will be given by the co-hom functor. 
Proof. See [38, Proposition 1.10].

Another instance in which the co-hom functor corresponding to $\mathrm{M}$, a quasi-finite right $C$-comodule, returns an object with additional structure to that of a vector space occurs when the co-hom functor is applied to $M$ itself. In this case $E:=h_{C}(M, M)$ can be given a coalgebra structure by defining $\Delta_{E}$ and $\varepsilon_{E}$ to be the unique maps satisfying

$$
\left(E \otimes \varphi_{M}\right) \circ \varphi_{M}=\left(\Delta_{E} \otimes M\right) \circ \varphi_{M}, \quad\left(\varepsilon_{E} \otimes M\right) \circ \varphi_{M}=M .
$$

That this produces a coalgebra structure can be checked in a similar way to which $\rho^{h_{C}(M, C)}$ is shown to be coaction in proposition 1.3.8 (a full proof of this can be found in [14, Subsection 12.9]).

Definition 1.3.13. Suppose $M$ is quasi-finite as a right $C$-comodule then $E:=h_{C}(M, M)$ with the coalgebra structure defined by the above maps, is called the coendomorphism coalgebra of $M$.

Proposition 1.3.14. Let $M$ be a quasi-finite right $C$-comodule and set $E=h_{C}(M, M)$ to be the coendomorphism coalgebra of $M$. Then $M$ is an $(E, C)$-bicomodule, with left coaction given by the unit of adjunction $\varphi_{M}: M \rightarrow h_{C}(M, M) \otimes M$. Furthermore if $M$ is also a $(D, C)$-bicomodule, then there exists an unique coalgebra map $\pi: E \rightarrow D$ such that the left $D$-coaction is equal to $(\pi \otimes M) \circ \varphi_{M}$.

Proof. From the defining properties of the coendomorphism coalgebra it is clear that $\varphi_{M}: M \rightarrow E \otimes M$ will be a left coaction and by the definition of $\varphi_{M}$ this map must be right $C$-colinear. Hence $M$ is an $(E, C)$-bicomodule with this left coaction. Now suppose $M$ is also a $(D, C)$-bicomodule. By the universal mapping property of Remark $1.3 .3,{ }^{M} \rho: M \rightarrow$ $D \otimes M$ can be factorized by the composition

$$
M \stackrel{\varphi_{M}}{\longrightarrow} E \otimes M \stackrel{\pi \otimes M}{\longrightarrow} D \otimes M,
$$

for a unique choice of $k$-linear map $\pi: E \rightarrow D$. Now observe

$$
\begin{aligned}
\left(\left(\Delta_{D} \circ \pi\right) \otimes M\right) \circ \varphi_{M} & =\left(\Delta_{D} \otimes M\right) \circ{ }^{M} \rho \\
& =\left(D \otimes{ }^{M} \rho\right) \circ{ }^{M} \rho \\
& =\left(D \otimes{ }^{M} \rho\right) \circ(\pi \otimes M) \circ \varphi_{M} \\
& =\left(\pi \otimes{ }^{M} \rho\right) \circ \varphi_{M} \\
& =\left(\pi \otimes\left((\pi \otimes M) \circ \varphi_{M}\right)\right) \circ \varphi_{M} \\
& =(\pi \otimes \pi \otimes M) \circ\left(E \otimes \varphi_{M}\right) \circ \varphi_{M} \\
& =(\pi \otimes \pi \otimes M) \circ\left(\Delta_{E} \otimes M\right) \circ \varphi_{M} \\
& =\left(\left((\pi \otimes \pi) \circ \Delta_{E}\right) \otimes M\right) \circ \varphi_{M} .
\end{aligned}
$$

Where the equalities follow by the coassociativity of ${ }^{M} \rho$ and the defining characteristics of the maps $\pi$ and $\Delta_{E}$. Hence the map $\pi$ as defined above is comultiplicative. Similarly it is clear that

$$
\left(\left(\varepsilon_{D} \circ \pi\right) \otimes M\right) \circ \varphi_{M}=\left(\varepsilon_{D} \otimes M\right) \circ{ }^{M} \rho=M=\left(\varepsilon_{E} \otimes M\right) \circ \varphi_{M},
$$

hence the map $\pi$ is also counital. 
Definition 1.3.15. A right $C$-comodule $M$ is called an injector if the tensor functor $-\otimes M$ : Vect $_{k} \rightarrow \mathbf{M}^{C}$ respects injective objects.

Proposition 1.3.16. If $M$ is quasi-finite as a right $C$-comodule then $M$ is an injector if and only if the co-hom functor is exact.

Proof. Suppose $V$ is an injective object in $\operatorname{Vect}_{k}$ and $h_{C}(M,-)$ is exact. Since $V$ is an injective object the contravariant functor $\operatorname{Hom}_{k}(-, V)$ is exact. Now observe this implies that the composition $\operatorname{Hom}_{k}\left(h_{C}(M,-), V\right)$ is exact and so by the adjointness isomorphism $\operatorname{Hom}^{-C}(-, V \otimes M)$ must also be exact. Therefore $V \otimes M$ is an injective object in $\mathbf{M}^{C}$. Conversely suppose that the tensor functor $-\otimes M$ : Vect ${ }_{k} \rightarrow \mathbf{M}^{C}$ respects injective objects. Since $h_{C}(M,-)$ is a left adjoint it is right exact, so it is only necessary to check that $h_{C}(M,-)$ preserves monomorphisms. Let $f: N \rightarrow L$ be a monomorphism and set $g=h_{C}(M, f)$. Now by the adjointness for all $V \in$ Vect $_{k}$ there is a commutative diagram

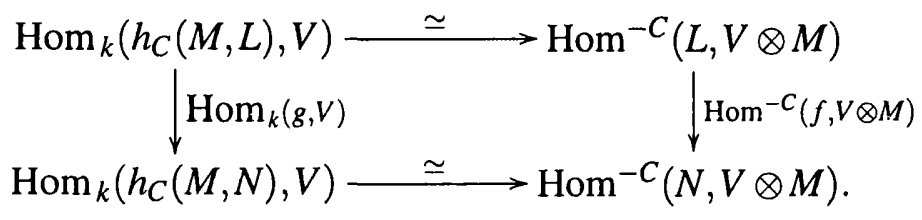

All categories of modules over rings have an injective cogenerator, and so in particular such a diagram exists for $V$ an injective cogenerator in Vect ${ }_{k}$. With this choice of $V$ and the assumption that the tensor functor preserves injective objects $\mathrm{Hom}^{-C}(-, V \otimes M)$ is exact and so $\operatorname{Hom}^{-C}(f, V \otimes M)$ is a surjection. By the diagram this means that $\operatorname{Hom}_{k}(g, V)$ is surjective. Now since $V$ was chosen to be a cogenerator this implies that $g=h_{C}(M, f)$ is a monomorphism. Therefore the co-hom functor is exact.

Remark 1.3.17. Suppose $M$ is a $(D, C)$-bicomodule which is quasi-finite as a right $C$ comodule. By the the previous proposition saying that $M$ is an injector as a right $C$ comodule is equivalent to saying that the functor $h_{C}(M,-): \mathbf{M}^{C} \rightarrow$ Vect $_{k}$ is exact. In view of Theorem 1.3.12 in this situation it is just as well to view the co-hom functor as $h_{C}(M,-): \mathbf{M}^{C} \rightarrow \mathbf{M}^{D}$. Now $h_{C}(M,-): \mathbf{M}^{C} \rightarrow \operatorname{Vect}_{k}$ is exact if and only if $h_{C}(M,-):$ $\mathbf{M}^{C} \rightarrow \mathbf{M}^{D}$ is exact. By a similar method to that used in the previous proposition it is possible to show that $h_{C}(M,-): \mathbf{M}^{C} \rightarrow \mathbf{M}^{D}$ is exact if and only if $-\square_{D} M: \mathbf{M}^{D} \rightarrow \mathbf{M}^{C}$ preserves injective objects. Hence the following statements are equivalent

1. $\mathrm{M}$ is an injector as a right $C$-comodule.

2. $h_{C}(M,-): \mathbf{M}^{C} \rightarrow$ Vect $_{k}$ is exact.

3. $-\square_{D} M: \mathbf{M}^{D} \rightarrow \mathbf{M}^{C}$ preserves injective objects.

4. $h_{C}(M,-): \mathbf{M}^{C} \rightarrow \mathbf{M}^{D}$ is exact.

Proposition 1.3.18. Suppose $M$ is a quasi-finite injector as a right $C$-comodule. Then the map $\delta_{N}$ (as defined in Proposition 1.3.11) is an isomorphism for every right $C$-comodule $N$. Moreover these isomorphisms are natural transformations, and hence form an isomorphism of functors

$$
h_{C}(M,-) \simeq-\square_{C} h_{C}(M, C),
$$


where $h_{C}(M, C)$ has the left coaction described in Proposition 1.3.7.

Proof. By the assumptions the co-hom functor preserves injective maps hence the rows of the following diagram are exact

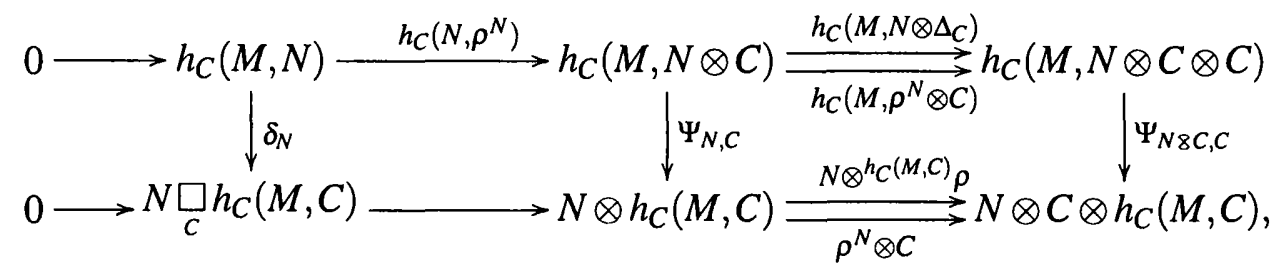

where the maps $\Psi$ are isomorphisms as defined in Proposition 1.3.5. Now a few straight forward calculations show that this diagram is commutative and since the maps $\Psi$ are isomorphisms so is the map $\delta_{N}$. For all right $C$-comodule maps $f: N \rightarrow L$, the diagram

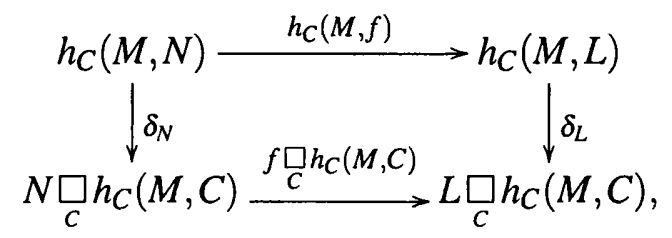

is commutative since

$$
\begin{aligned}
\left(\left(\delta_{L} \circ h_{C}(M, f)\right) \otimes M\right) \circ \varphi_{N} & =\left(\delta_{L} \otimes M\right) \circ \varphi_{L} \circ f \\
& =\left(L \square \varphi_{C}\right) \circ \rho^{L} \circ f \\
& =\left(L \square_{C} \varphi_{C}\right) \circ(f \square C) \circ \rho^{N} \\
& =\left(f \square_{C} h_{C}(M, C) \otimes M\right) \circ\left(N \square_{C} \varphi_{C}\right) \circ \rho^{N} \\
& =\left(\left(\left(f \square_{C} h_{C}(M, C)\right) \circ \delta_{N}\right) \otimes M\right) \circ \varphi_{N} .
\end{aligned}
$$

The above equalities follow by the repeated use of the defining characteristics of the maps $\delta$ and $h_{C}(M, f)$, and the right $C$-colinearity of $f$. Hence the result follows by the universal mapping property of Remark 1.3.3.

This completes our introduction to the theory of quasi-finite injectors. In chapter 3 we will return to the results we have mentioned here to study a special class of quasi-finite comodules. 


\section{Chapter 2}

\section{Corings and weak entwining structures}

\subsection{Corings}

\subsubsection{Introduction}

The concept of a coring is a generalization of a coalgebra. It is an interesting structure as many results which can be proven for coalgebras over commutative rings hold in this more general setting. Also the concept of a coring unifies many other algebraic structures. Finally there are situations in which one is drawn to consider such a structure, for example in investigating ring extensions. In fact it was in this context in which corings were first seriously studied as algebraic objects, see [37].

Definition 2.1.1. An $A$-coring is an (A,A)-bimodule $\mathfrak{C}$ together with two $(A, A)$-bimodule maps $\varepsilon_{\mathfrak{C}}: \mathfrak{C} \rightarrow A$ and $\Delta_{\mathfrak{C}}: \mathfrak{C} \rightarrow \mathfrak{C} \otimes \underset{A}{\otimes}$ which render the diagrams
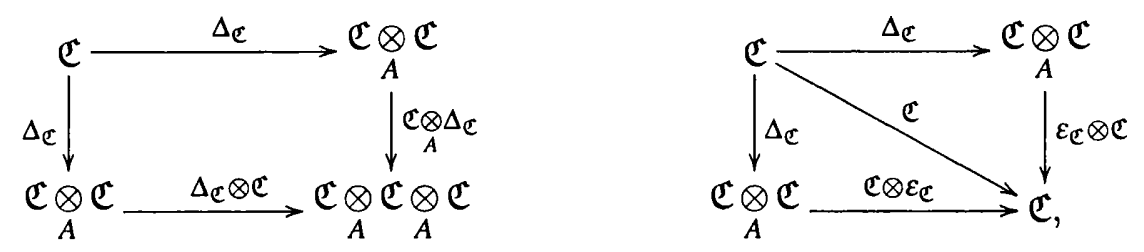

commutative. The map $\varepsilon_{\mathfrak{C}}$ is referred to as the counit and map $\Delta_{\mathfrak{C}}$ as the coproduct. A morphism of $A$-corings is a map $f: \mathfrak{C} \rightarrow \mathfrak{D}$ satisfying

$$
(f \underset{A}{\otimes} f) \circ \Delta_{\mathfrak{C}}=\Delta_{\mathfrak{D}} \circ f, \quad \varepsilon_{\mathfrak{D}} \circ f=\varepsilon_{\mathfrak{C}}
$$

Example 2.1.2. To any algebra map $f: B \rightarrow A$ it is possible to associate an $A$-coring as follows. First define $\mathfrak{C}=A \underset{B}{\otimes} A$ where $A$ is viewed as a $(B, B)$-bimodule via the map $f$ and view $\mathfrak{C}$ as an $(A, A)$-bimodule in the obvious way. Then set

$$
\begin{gathered}
\Delta_{\mathfrak{C}}: \mathfrak{C} \rightarrow \underset{A}{\mathbb{C}} \underset{A}{\mathbb{C}}, \quad a \underset{B}{\otimes} a^{\prime} \mapsto a \underset{B}{\otimes} \underset{A}{\otimes} \underset{B}{\otimes} \otimes_{B} a^{\prime} \\
\varepsilon_{\mathfrak{C}}: \mathfrak{C} \rightarrow A, \quad a \underset{B}{\otimes} a^{\prime} \mapsto a a^{\prime} .
\end{gathered}
$$

An A-coring arising in this way is called a Sweedler A-coring (see [37]). 
Generalizing these most fundamental examples of A-corings, are the following type of A-corings introduced in [23].

Example 2.1.3. Given a $(B, A)$-bimodule $M$ that is finitely generated and projective as a right $A$-module, set $M^{*}:=\operatorname{Hom}_{A}(M, A)$ to be the dual $(A, B)$-module. Then letting $\left\{e_{i} \in\right.$ $\left.M, e_{i}^{*} \in M^{*}\right\}_{i \in I}$ be some choice of finite dual basis of $M$, it is possible to define an $A$-coring

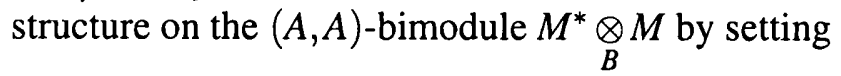

$$
\begin{gathered}
\Delta_{M^{*} \otimes}^{\otimes}: M_{B}^{*} \underset{B}{\otimes} M \rightarrow M_{B}^{*} \underset{B}{\otimes} \underset{A}{\otimes} M^{*} \underset{B}{\otimes} M, \quad \phi \otimes m \mapsto \sum_{i \in I} \phi \otimes e_{i} \otimes e_{i}^{*} \otimes m, \\
\varepsilon_{M^{*} \otimes M}^{\otimes}: M_{B}^{*} \underset{B}{\otimes} M \rightarrow A, \quad \phi \otimes m \mapsto \phi(m) .
\end{gathered}
$$

An A-coring arising in this way is called a comatrix A-coring. It is shown in [23] that for such an $A$-coring the coproduct is independent of the choice of finite dual basis.

The notion of comodules for a coalgebra can be generalized to $A$-coring as follows.

Definition 2.1.4. A comodule for an $A$-coring is a pair $\left(M, \rho^{M}\right)$ where $M$ is a right $A$ module and $\rho^{M}: M \rightarrow M \underset{A}{\otimes} \mathfrak{C}$ is a map of right $A$-modules satisfying

$$
\left.\left(\rho^{M} \underset{A}{\otimes} \mathfrak{C}\right) \circ \rho^{M}=\left(\underset{A}{\otimes} \Delta_{\mathfrak{C}}\right) \circ \rho^{M}, \quad \underset{A}{\left(M \otimes \varepsilon_{\mathfrak{C}}\right.}\right) \circ \rho^{M}=M .
$$

The map $\rho^{M}$ is referred to as a right $\mathfrak{C}$-coaction and just as for comodules the above conditions are referred to as coassociativity and counitality. The category of all such objects with morphisms $f: M \rightarrow N$ that are right $A$-linear and right $\mathfrak{C}$-colinear (in the sense that $\left.(f \otimes \mathfrak{C}) \circ \rho^{M}=\rho^{N} \circ f\right)$ is denoted $\mathbf{M}^{\mathfrak{C}}$.

Example 2.1.5. Suppose $M$ is a right $\mathfrak{C}$-comodule that is finitely generated and projective as a right $A$-module. Then a left $\mathfrak{C}$-module structure can be defined on $M^{*}=\operatorname{Hom}_{-A}(M, \mathfrak{C})$ by the composition of maps

$$
M^{*} \rightarrow \operatorname{Hom}_{-A}(M, \mathfrak{C}) \rightarrow \underset{A}{\mathfrak{C}} \otimes M^{*}
$$

described for some choice of dual basis $\left\{e_{i} \in M, e_{i}^{*} \in M^{*}\right\}_{i \in I}$ as follows

$$
g \mapsto(\underset{A}{\otimes} \mathfrak{C}) \circ \rho^{M} \mapsto \sum_{i \in I} g\left(e_{i[0]}\right) e_{i[1]} \underset{A}{\otimes} e_{i}^{*},
$$

where $\rho^{M}\left(e_{i}\right)=\sum e_{i[0]} \underset{A}{\otimes} e_{i[1]}$. See [14, Subsection 19.14] for a more detailed account of these constructions. It will also be useful to note that there is a natural isomorphism between $\operatorname{End}^{-\mathfrak{C}}(M)$ and End ${ }^{\mathfrak{C}-}\left(M^{*}\right)$ which we shall denote by $\Gamma_{M}: \operatorname{End}^{-\mathfrak{C}}(M) \rightarrow \operatorname{End}^{\mathfrak{C}-}\left(M^{*}\right)$. For a choice of dual basis $\left\{e_{i} \in M, e_{i}^{*} \in M^{*}\right\}_{i \in I}, s \in \operatorname{End}^{-\mathfrak{C}}(M), \xi \in M^{*}, t \in \operatorname{End}^{\mathfrak{C}-}\left(M^{*}\right)$ and $m \in M$

$$
\Gamma_{M}(s)(\xi):=\sum_{i \in I} \xi\left(s\left(e_{i}\right)\right) e_{i}^{*}=\xi \circ s, \quad \Gamma_{M}^{-1}(t)(m)=\sum_{i \in I}(t)(m)=\sum_{i \in I} e_{i} t\left(e_{i}^{*}\right)(m) .
$$
$\xi \circ s$.

In this way one views $M^{*}$ as a right $\operatorname{End}^{-\mathfrak{C}}(M)$-module with the action $\xi_{s}=\Gamma_{M}(s)(\xi)=$ 
We shall now highlight the unifying power of the theory of $A$-corings by showing how concepts relating to entwining structures can be viewed as special cases of the theory of $A$-corings. This was first noticed by Takeuchi, and was the main cause of the recent resurgence of interest in corings.

Proposition 2.1.6. For an entwining structure $(A, C, \psi)$ it is possible to define an $A$-coring structure on $\mathfrak{C}:=A \otimes C$ as follows. First view $\mathfrak{C}$ as an $(A, A)$-bimodule by setting $a \cdot\left(a^{\prime} \otimes\right.$ c) $\cdot a^{\prime \prime}:=a a^{\prime} \psi\left(c \otimes a^{\prime \prime}\right)$ and then define $\Delta_{\mathfrak{C}}: \mathfrak{C} \rightarrow \mathfrak{C} \otimes \mathfrak{C} \simeq A \otimes C \otimes C, \quad \Delta_{\mathfrak{C}}:=A \otimes \Delta_{C}$ and $\varepsilon_{\mathfrak{C}}=A \otimes \varepsilon_{C}$. Conversely if the the maps above define an $A$-coring structure on $A \otimes C$ then $(A, C, \psi)$ must be an entwining structure. Furthermore under this correspondence $\mathbf{M}^{\mathfrak{C}}=\mathbf{M}(\psi)_{A}^{C}$.

Proof. See [4, Proposition 2.2].

\subsubsection{Galois corings and comodules}

With the previous proposition in mind it is possible to define a special type of $A$-coring which generalizes $A$-corings which are constructed in the way described in Proposition 2.1.6, from the canonical entwining structure associated to a $C$-Galois extension $B \subseteq A$. These constructions first appeared in [4]. As background to these constructions we will first investigate how the notion of a grouplike element in coalgebra generalizes to $A$-corings. This idea was originally developed by Sweedler in [37].

Definition 2.1.7. An element $g$ of an $A$-coring $\mathfrak{C}$ is said to be grouplike if $\Delta_{\mathfrak{C}}(g)=g \underset{A}{\otimes} g$ and $\varepsilon(g)=1$.

For such elements there is the following result [37, Proposition 1.9].

Proposition 2.1.8. Let $\mathfrak{C}$ be the Sweedler A-coring associated to an extension $\phi: B \rightarrow A$ then

(a) $1 \underset{B}{\otimes} 1$ is grouplike element of $\mathfrak{C}$.

(b) Let $\mathfrak{D}$ be some other $A$-coring and let $G_{\phi}(\mathfrak{D})$ denote the set of grouplike elements, $g$, in $\mathfrak{D}$ satisfying $\phi(b) g=g \phi(b)$ for all elements of $B$. For each $g \in G_{\phi}(\mathfrak{D})$ there exists a unique A-coring map $\zeta: \mathfrak{C} \rightarrow \mathfrak{D}$ such that $\zeta\left(1 \otimes_{B} 1\right)=g$. Conversely for each $A$-coring map $\xi: \mathfrak{C} \rightarrow \mathfrak{D}$, the element $\xi(1 \underset{B}{\otimes} 1) \in G_{\phi}(\mathfrak{D})$.

Also as regards the comodules of an $A$-coring we have the following correspondence

Proposition 2.1.9. For any $A$-coring $\mathfrak{C}$, there is a one to one correspondence between right $\mathfrak{C}$ coactions on $A$ and grouplike elements in $\mathfrak{C}$.

Proof. The correspondence goes as follows: given a right $\mathfrak{C}$-coaction on $A$, the element $\rho^{\mathfrak{C}}(1)$ is grouplike. Conversely given a grouplike element $g \in \mathfrak{C}$, set $\rho^{A}(a):=1 \underset{A}{\otimes} g a$. See [4, Lemma 5.1] for details. 
Now given an entwining $\psi$ that arises from a $C$-Galois extension $B \subseteq A$, let $\mathfrak{D}$ denote the associated $A$-coring. Since $A \in \mathbf{M}(\psi)_{A}^{C}=M^{\mathfrak{D}}$ there is a distinguished grouplike element $g \in \mathfrak{D}$. Furthermore letting $\phi: B \rightarrow A$ be the canonical inclusion of the coinvariant subalgebra we obtain that $g \in G_{\phi}(\mathfrak{D})$. So by Proposition 2.1 .8 for the Sweedler coring $\mathfrak{C}$ associated to $\phi$ there is a unique coring map $\xi: \mathfrak{C} \rightarrow \mathfrak{D}$ such that $\xi(1 \underset{B}{\otimes} 1)=g$. In this situation it turns out that $\xi$ is an isomorphism. Removing the requirement that $\mathfrak{D}$ arises in this way one arrives at the following entwining free definition.

Definition 2.1.10. Let $\mathfrak{C}$ be an $A$-coring with a grouplike element $g$ and define $B:=A^{\text {coC }}=$ $\{b \in A \mid b g=g b\}$. $\mathfrak{C}$ is said to be Galois (with respect to $g$ ) if the map $\xi: A \otimes A \rightarrow \mathfrak{C}$ uniquely defined by the condition $\xi(1 \underset{A}{\otimes} 1)=g$ is an isomorphism of $A$-corings.

By the considerations already mentioned the $A$-coring arising from a $C$-Galois extension via the canonical entwining is a Galois coring. Furthermore the converse is true. This is described in the following example from [4].

Example 2.1.11. Let $(A, C, \psi)$ be a (right-right) entwining structure and let $\mathfrak{C}=A \otimes C$ be the associated $A$-coring. Then $\mathfrak{C}$ is a Galois coring if and only if $(A, C, \psi)$ is the canonical entwining structure of a $C$-Galois extension.

Continuing this chain of generalizations, in order to obtain further insight into Galois corings it has proved productive to study special types of comodules of an A-coring. This approach started in [23] and was further investigated in [5].

Definition 2.1.12. Let $\mathfrak{C}$ be an $A$-coring, $M \in \mathbf{M}^{\mathfrak{C}}$ and set $S:=\operatorname{End}^{-\mathfrak{C}}(M)$. Then $M$ is said to be a Galois (right) comodule provided that it is finitely generated and projective as a right $A$-module, and the evaluation map

$$
\varphi_{\mathfrak{C}}: \operatorname{Hom}^{-\mathfrak{C}}(M, \mathfrak{C}) \underset{S}{\otimes} M \rightarrow \mathfrak{C}, \quad f \otimes m=f(m),
$$

is an isomorphism of right $\mathfrak{C}$-comodules, where $\mathfrak{C}$ is viewed as a right $\mathfrak{C}$-comodule in the obvious way via its coproduct.

The map $\varphi$ above can been seen to have its origins as the counit of the pair of adjoint functors $\left(-\underset{S}{\otimes} M, \operatorname{Hom}^{-\mathfrak{C}}(M,-)\right)$, for which the counit is the evaluation map and unit is given by the map $x \mapsto[x \mapsto x \otimes m]$. Equivalently such comodules can be defined in a way which is easily seen to generalize Galois $A$-corings. First observe that $M$ as described above is an $(S, A)$-bimodule and $\operatorname{Hom}^{-\mathfrak{C}}(M, \mathfrak{C}) \simeq M^{*}$. Studying the situation in this way one observes it is possible to make the following equivalent definition.

Definition 2.1.13. Let $\mathfrak{C}$ be an $A$-coring, $M \in \mathbf{M}^{\mathfrak{C}}$ and set $S:=\operatorname{End}^{-\mathfrak{C}}(M)$. Suppose $M$ is finitely generated projective as a right $A$-module then $M$ is $(S, A)$-bimodule and $M^{*} \underset{S}{\otimes} M$ can be viewed as a comatrix $A$-coring as described in Example 2.1.3. Now there is a natural $A$-coring map

$$
\operatorname{can}_{\mathrm{M}}: \mathrm{M}^{*} \underset{\mathrm{S}}{\otimes \mathrm{M}} \rightarrow \mathfrak{C}, \quad \xi \otimes \mathrm{m} \mapsto \sum \xi\left(\mathrm{m}_{[0]}\right) \mathrm{m}_{[1]}
$$

$\mathrm{M}$ is a Galois comodule if this map is an isomorphism. 
Remark 2.1.14. Now with this definition it will be useful to observe that in the special case when $M=A$ that the corresponding comatrix $A$-coring is precisely the Sweedler $A$ coring of the inclusion of the subalgebra of coinvariants $A^{\text {coC }}$. To see this, first note that each $f \in \operatorname{End}^{-\mathfrak{C}}(A)$ is right $A$-linear and therefore uniquely determined by its value at 1 . Then note that any right $A$-linear map $g: A \rightarrow A$ uniquely determined by the condition that $g(1)=b$ is right $\mathfrak{C}$-colinear if and only if $b \in A^{\text {coC }}$. It is then easily seen that $A^{\text {coC }}$ is isomorphic to $S=\mathrm{End}^{-\mathfrak{C}}(A)$. In view of this, it is clear that $A$ is a Galois $\mathfrak{C}$-comodule if and only if $\mathfrak{C}$ is a Galois $A$-coring.

The main properties of such comodules are described in the following theorem first stated in [23, Theorem 3.2].

Theorem 2.1.15. (The Galois comodule structure theorem) Let $\mathfrak{C}$ be an A-coring and let $M$ be a right $\mathfrak{C}$-comodule which is finitely generated projective as a right $A$-module. Set $S=\operatorname{End}^{-\mathfrak{C}}(M)$.

(a) The following are equivalent:

(1) $M$ is Galois comodule which is flat as a left S-module.

(2) $\mathfrak{C}$ is a flat left A-module and $M$ is a generator in $\mathbf{M}^{\mathfrak{C}}$.

(3) $\mathfrak{C}$ is flat left $A$-module and, for any $N \in \mathbf{M}^{\mathfrak{C}}$, the counit of adjunction $\varphi_{N}$ : $\operatorname{Hom}^{-\mathfrak{C}}(M, N) \underset{S}{\otimes} M \rightarrow N$ is an isomorphism of right $\mathfrak{C}$-comodules.

(b) The following are equivalent:

(1) $M$ is a Galois comodule which is faithfully flat as a left S-module.

(2) $\mathfrak{C}$ is a flat left A-module and $M$ is a projective generator in $\mathbf{M}^{\mathfrak{C}}$.

(3) $\mathfrak{C}$ is a flat left $A$-module and $\operatorname{Hom}^{-\mathfrak{C}}(M,-): \mathbf{M}^{\mathfrak{C}} \rightarrow \mathbf{M}_{S}$ is an equivalence with inverse $-\underset{S}{\otimes} M: \mathbf{M}_{S} \rightarrow M^{\mathfrak{C}}$.

Proof. See [14, Subsections 18.23 and 18.27].

We have already seen how it is possible to associate an $A$-coring to a $C$-Galois extension, how Galois $A$-corings generalize such an object and then continuing the chain of generalization introduced Galois comodules. Now as a model for non-commutative principal bundles, a particular type of $C$-Galois extension termed a principal $C$-extension has been introduced in [6]. This notion generalizes that of a faithfully flat Hopf-Galois extension with a bijective antipode and is a mild generalization in that it enjoys many of the same nice properties. Making a chain of generalizations for these principal $C$-extensions, as has already been demonstrated for $C$-Galois extensions, one can arrive at a special class of $\mathrm{Ga}$ lois comodules. These comodules generalize faithfully flat Hopf-Galois extensions with bijective antipode, yet still admit a theorem similar to what is referred to as Schneider's Theorem 1, as stated in [34]. We first recall Schneider's result.

Theorem 2.1.16. Let $H$ be a Hopf algebra over a field. Assume the antipode of $H$ is bijective and $B \subseteq A$ is a $H$-Galois extension. Then the following are equivalent:

(a) (1) $A$ is injective as a right $H$-comodule;

(2) $\operatorname{can}_{A}: A \underset{B}{\otimes} A \rightarrow A \otimes H, a \otimes a^{\prime} \mapsto \sum a a_{[0]}^{\prime} \otimes a_{[1]}$ is surjective. 
(b) $\mathbf{M}_{B} \rightarrow \mathbf{M}_{A}^{H}, M \mapsto M \underset{B}{\otimes} A$, is an equivalence.

(c) ${ }_{B} \mathbf{M} \rightarrow{ }_{A} \mathbf{M}^{H}, M \mapsto \underset{B}{A} \underset{B}{\otimes}$, is an equivalence.

(d) (1) A is faithfully flat as a left B-module;

(2) can $_{A}$ is an isomorphism.

(e) (1) A is faithfully flat as a right B-module;

(2) can $_{A}$ is an isomorphism.

Now this result can be generalized as follows. For completeness we provide a proof for algebras over a commutative ring. For algebras over a field this is precisely [5, Theorem 4.4].

Theorem 2.1.17. Let $\mathfrak{C}$ be an $A$-coring and $M$ a right $\mathfrak{C}$-comodule that is finitely generated and projective as a right $A$-module. Let $S=\operatorname{End}^{-\mathfrak{C}}(M)$. Suppose that:

(a) the map

$$
\chi: S \otimes M \rightarrow \operatorname{Hom}^{\mathfrak{C}-}\left(M^{*}, M^{*} \otimes M\right), \quad b \otimes m \mapsto[\xi \mapsto \xi \circ b(-) \otimes m],
$$

is an isomorphism of left S-modules;

(b) the map

$$
\widetilde{\operatorname{can}_{M}}: M^{*} \otimes M \rightarrow \mathfrak{C}, \quad \xi \otimes m \mapsto \sum \xi\left(m_{[0]}\right) m_{[1]},
$$

is a split epimorphism of left $\mathfrak{C}$-comodules.

Then $M$ is a Galois comodule and $M$ is a $k$-relatively projective left $S$-module (meaning that any $S$-module epimorphism $N \rightarrow M$ that splits as an $k$-module map splits as a $S$-module map).

Proof. First observe that $\operatorname{Hom}^{\mathfrak{C}-}\left(M^{*}, \mathfrak{C}\right) \simeq{ }^{*}\left(M^{*}\right) \simeq M$, where the first isomorphism is an example of a Hom-tensor relation for left $\mathfrak{C}$-comodules (see [14, Subsection 18.27]) explicitly, for general $L, N \in{ }^{\mathfrak{C}} M$ and $X \in{ }_{A} M$,

$$
\operatorname{Hom}^{\mathfrak{C}-}(N, \mathfrak{C} \underset{A}{\otimes} X) \rightarrow \operatorname{Hom}_{A-}(N, X), \quad f \mapsto\left(\varepsilon_{\mathfrak{C}} \underset{A}{\otimes X} X\right) \circ f .
$$

The second of the isomorphisms, $M \simeq{ }^{*}\left(M^{*}\right)$, is given by, for each $m \in M$, evaluating elements of $M^{*}$ at $m$. This produces a natural map $M \rightarrow \operatorname{Hom}_{A-}\left(\operatorname{Hom}_{-A}(M, A), A\right)$ which must be by bijective since $M$ is finitely generated projective. Now in view of assumption (b) $\mathfrak{C}$ is a direct summand of $M^{*} \otimes M$, by the isomorphism in (a) and the isomorphism described above, this then means that $\operatorname{Hom}^{\mathfrak{C}-}\left(M^{*}, \mathfrak{C}\right) \simeq M$ is a direct summand of the left $S$ module $\mathrm{Hom}^{\mathfrak{C}-}\left(M^{*}, M^{*} \otimes M\right) \simeq S \otimes M$. Now observe that $S \otimes M$ is a k-relatively projective left $S$-module, since given any $N \in{ }_{S} \mathbf{M}$ and left $S$-module epimorphism $f: N \rightarrow S \otimes M$, with a $k$-linear splitting $\hat{v}$, it is easily verified that $v: S \otimes M \rightarrow N$ given by the formula $v(s \otimes m)=s \hat{v}(1 \otimes m)$, is a left $S$-linear splitting of $f$. Therefore, since $S \otimes M$ is a $k$-relatively projective left $S$-module, so is $M$.

For the adjoint pair of functors

$$
M^{*} \underset{S}{\otimes}-:{ }_{s} \mathbf{M} \rightarrow{ }^{\mathfrak{C}} \mathbf{M}, \quad \operatorname{Hom}^{\mathfrak{C}-}\left(M^{*},-\right):{ }^{\mathfrak{C}} \mathbf{M} \rightarrow s^{\mathbf{M}}
$$


the counit of the adjunction

$$
\widehat{\varphi}_{M^{*} \otimes M}: M^{*} \underset{S}{\otimes \operatorname{Hom}^{\mathfrak{C}}-}\left(M^{*}, M^{*} \otimes M\right) \rightarrow M^{*} \otimes M, \quad m^{*} \underset{S}{\otimes} f \mapsto f\left(m^{*}\right)
$$

can be factored as the following composition of maps

$$
M^{*} \underset{S}{\otimes} \operatorname{Hom}^{\mathfrak{C}-}\left(M^{*}, M^{*} \otimes M\right) \stackrel{M^{*} \otimes \chi^{-1}}{\longrightarrow} M^{*} \underset{S}{\otimes} S \otimes M \stackrel{\cong}{\longrightarrow} M^{*} \otimes M .
$$

Hence $\widehat{\varphi}_{M^{*} \otimes M}$ is an isomorphism. Using the naturality of $\widehat{\varphi}$ it is possible to produce a diagram

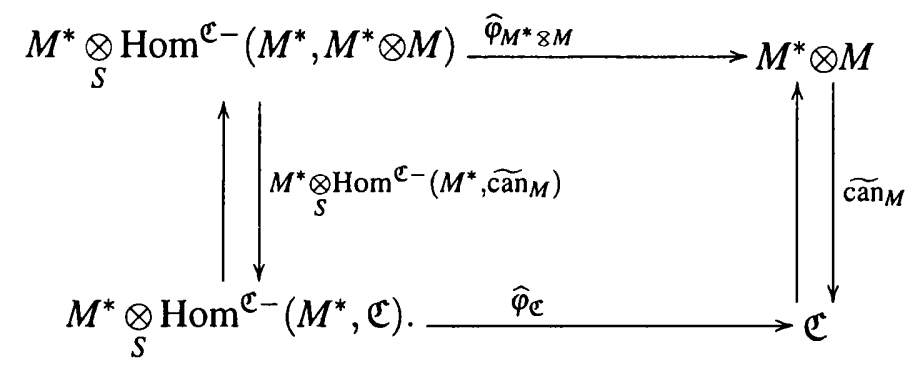

Where the upward pointing arrows are sections of $M^{*} \otimes{ }_{S} \mathrm{Hom}^{\mathfrak{C}-}\left(M^{*}, \widetilde{\mathrm{can}}_{M}\right)$ and $\widetilde{\mathrm{can}_{M}}$ respectively. The existence of these maps is guaranteed by assumption (b). Since $\widehat{\varphi}_{M^{*} \otimes M}$ is an isomorphism, the map $\widehat{\varphi}_{\mathfrak{C}}$ is bijective (it is a $k$-linear isomorphism). Using the identifications $\operatorname{Hom}^{\mathfrak{C}-}\left(M^{*}, \mathfrak{C}\right) \simeq M$ and $M^{*} \simeq \operatorname{Hom}^{-\mathfrak{C}}(M, \mathfrak{C})$ there is a $k$-linear isomorphism $M^{*} \otimes_{S} \mathrm{Hom}^{\mathfrak{C}-}\left(M^{*}, \mathfrak{C}\right) \simeq \operatorname{Hom}^{-\mathfrak{C}}(M, \mathfrak{C}) \otimes_{S} M$, and using this isomorphism we can construct a commutative diagram

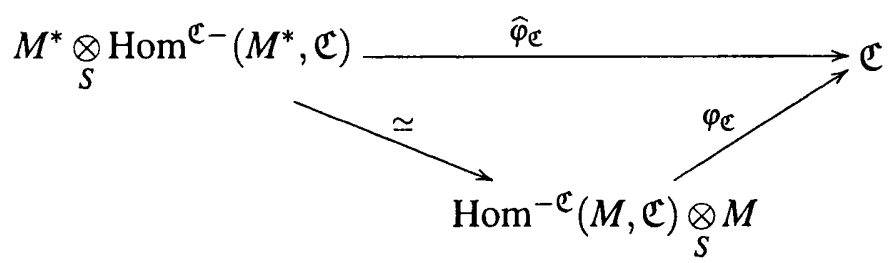

from which we can deduce that since $\widehat{\varphi}_{\mathfrak{C}}$ is bijective, so is $\varphi_{\mathfrak{C}}$. Therefore since $\varphi_{\mathfrak{C}}$ is a bijective morphism in $M^{\mathfrak{C}}$, it must an isomorphism of right $\mathfrak{C}$-comodules, so we conclude that $M$ is a Galois right $\mathfrak{C}$-comodule.

In the case of algebras over a field, which is of primary interest, to non-commutative geometry for example, the special class of Galois comodules which satisfy the assertions of Theorem 2.1.17 is described as follows.

Definition 2.1.18. A Galois (right) comodule $M$ is called a principal comodule provided $M$ is projective as a left $S:=$ End $^{-\mathfrak{C}}(M)$ module.

This definition is equally valid for algebras over a commutative ring, and it is possible to obtain a generalized version of [5, Theorem 4.4]. We are able to realize such a result as corollary of the previous theorem as follows. 
Corollary 2.1.19. Let $\mathfrak{C}$ be an $A$-coring and $M$ a right $\mathfrak{C}$-comodule that is finitely generated and projective as a right A-module. Let $S=\operatorname{End}^{-\mathfrak{C}}(M)$. Suppose that:

(a) $M$ is a projective $k$-module;

(b) the map

$$
\widetilde{\operatorname{can}}_{M}: M^{*} \otimes M \rightarrow \mathfrak{C}, \quad \xi \otimes m \mapsto \sum \xi\left(m_{[0]}\right) m_{[1]},
$$

is a split epimorphism of left $\mathfrak{C}$-comodules.

Then $M$ is a principal comodule.

Proof. It is well known that for any left $A$-module $N$ which if finitely generated projective and $\mathrm{k}$-module $V$ the map

$$
\theta: \operatorname{End}_{A-}(N) \otimes V \rightarrow \operatorname{Hom}_{A-}(N, N \otimes V), \quad t \otimes v \mapsto t(-) \otimes v
$$

is a $k$-isomorphism. The inverse of $\theta$ can be described in terms of a choice of dual basis $\left\{e_{i} \in N,{ }^{*} e_{i} \in{ }^{*} N\right\}_{i \in I}$ of ${ }_{A} N$ as follows, for all $f \in \operatorname{Hom}_{A-}(N, N \otimes V)$, writing $f(n)=$ $\sum f(n)^{[1]} \otimes f(n)^{[2]} \in N \otimes V$

$$
\theta^{-1}(f)=\sum_{i \in I}^{*} e_{i}(-) f\left(e_{i}\right)^{[1]} \otimes f\left(e_{i}\right)^{[2]}
$$

We now consider such a map $\theta$ with $V=M$ and $N=M^{*}$. By the definition of $\theta$ it is clear that it maps elements of End ${ }^{\mathfrak{C}-}\left(M^{*}\right) \otimes M$ to left $\mathfrak{C}$-colinear maps. We shall now show that, going in the other direction, $\theta^{-1}$ will map left $\mathfrak{C}$-colinear maps into $\operatorname{End}^{\mathfrak{C}-}\left(M^{*}\right) \otimes M$. Since $M$ is a projective k-module it must also be flat as a $k$-module, hence we are able to check if $\xi \otimes m$ is an element of End ${ }^{\mathfrak{C}-}\left(M^{*}\right) \otimes M$ as follows. First define a map

$$
\delta: \operatorname{End}_{A-}\left(M^{*}\right) \rightarrow \operatorname{Hom}_{A-}\left(M^{*}, \underset{A}{\otimes} \otimes\right)
$$

by

$$
\delta(\xi)(x):=\sum x_{[-1]} \underset{A}{\otimes} \xi\left(x_{[0]}\right)-\sum(\xi(x))_{[-1]} \underset{A}{\otimes}(\xi(x))_{[0]} .
$$

Where $\sum x_{[-1]} \otimes x_{[0]}$ is the result of applying the left $\mathfrak{C}$-coaction on $x \in M^{*}$, as described in Example 2.1.5. With this definition $\xi \in \operatorname{End}^{\mathfrak{C}-}\left(M^{*}\right) \subseteq \operatorname{End}_{A-}\left(M^{*}\right)$ if and only if $\xi \in$ $\operatorname{ker}(\delta)$. By the flatness of $M \operatorname{ker}(\delta \otimes M)=\operatorname{ker}(\delta) \otimes M$. Hence to check $\theta^{-1}(f) \in$ End $^{\mathfrak{C}-}\left(M^{*}\right) \otimes M$ all that is required is to verify that $(\delta \otimes M) \circ \theta^{-1}(f)=0$. For any left $\mathfrak{C}$-colinear map $f: M^{*} \rightarrow M^{*} \otimes M$ and $x \in M^{*}$, we can compute

$$
\begin{aligned}
x_{[-1]} \otimes \theta^{-1}(f)\left(x_{[0]}\right) & =\sum_{i \in I} x_{[-1]} \otimes{ }^{*} e_{i}\left(x_{[0]}\right) f\left(e_{i}\right)^{[1]} \otimes f\left(e_{i}\right)^{[2]} \\
& =\sum_{i \in I} x_{[-1]} \otimes f\left(^{*} e_{i}\left(x_{[0]}\right) e_{i}\right)^{[1]} \otimes f\left(^{*} e_{i}\left(x_{[0]}\right) e_{i}\right)^{[2]} \\
& =\sum_{i \in I} x_{[-1]} \otimes f\left(x_{[0]}\right)^{[1]} \otimes f\left(x_{[0]}\right)^{[2]} \\
& =\sum f(x)^{[1]}{ }_{[-1]} \otimes f(x)^{[1]}[0] \otimes f(x)^{[2]} .
\end{aligned}
$$


Where the second equality follows by the left $A$-linearity of $f$, the third by the properties of a dual basis and the last equality by the assumption that $f$ is left $\mathfrak{C}$-colinear. Likewise

$$
\begin{aligned}
\left(M^{*} \rho \otimes M\right) \circ \theta^{-1}(f)(x) & =\sum_{i \in I}\left({ }^{*} e_{i}(x) f\left(e_{i}\right)^{[1]}\right)_{[-1]} \otimes\left({ }^{*} e_{i}(x) f\left(e_{i}\right)^{[1]}\right)_{[0]} \otimes f\left(e_{i}\right)^{[2]} \\
& =\sum_{i \in I}\left(f\left({ }^{*} e_{i}(x) e_{i}\right)^{[1]}\right)_{[-1]} \otimes\left(f\left({ }^{*} e_{i}(x) e_{i}\right)^{[1]}\right)_{[0]} \otimes f\left({ }^{*} e_{i}(x) e_{i}\right)^{[2]} \\
& =\sum f(x)^{[1]}{ }_{[-1]} \otimes f(x)^{[1]}{ }_{[0]} \otimes f(x)^{[2]} .
\end{aligned}
$$

So $(\delta \otimes M) \circ \theta^{-1}(f)=0$. Now it clear that $\theta$ restricts to a $k$-isomorphism End $^{\mathfrak{C}-}\left(M^{*}\right) \otimes$ $M \simeq \operatorname{Hom}^{\mathfrak{C}-}\left(M^{*}, M^{*} \otimes M\right)$. Now using the isomorphism

$$
\Gamma_{M}: S=\operatorname{End}^{-\mathfrak{C}}(M) \rightarrow \operatorname{End}^{\mathfrak{C}-}\left(M^{*}\right),
$$

as described in (2.1), we can construct is a chain of isomorphisms

$$
\begin{gathered}
S \otimes M \stackrel{\Gamma_{M} \otimes M}{\longrightarrow} \text { End }^{\mathfrak{C}-}\left(M^{*}\right) \otimes M \stackrel{\theta}{\rightarrow} \operatorname{Hom}^{\mathfrak{C}-}\left(M^{*}, M^{*} \otimes M\right) \\
s \otimes m \mapsto[\xi \mapsto \xi \circ s] \otimes m \mapsto[\xi \mapsto \xi \circ s(-) \otimes m]
\end{gathered}
$$

This is precisely condition (a) of Theorem 2.1.17. Now since $M$ satisfies the conditions of this theorem $M$ is a Galois comodule and $M$ is a $k$-relatively projective left $S$-module. Since $M$ is a $k$-relatively projective left $S$ module and $M$ is a projective $k$-module, $M$ is a projective $S$-module, hence a principal comodule as required.

\subsection{Weak entwining structures}

\subsubsection{Motivation}

In certain topics in mathematical physics, for example in the theory of operator algebras and monoidal categories, it has been useful to consider an algebraic structure which is a generalization of a bialgebra. Following [3] and [30] the axioms for this object are as follows.

Definition 2.2.1. A weak $k$-bialgebra $H$ is a $k$-module with a $k$-algebra structure $(\mu, 1)$ and a $k$-coalgebra structure $(\Delta, \varepsilon)$, such that $\Delta$ is a multiplicative map and

$$
\begin{gathered}
\Delta^{2}(1)=\sum 1_{(1)} \otimes 1_{(2)} 1_{\left(1^{\prime}\right)} \otimes 1_{\left(2^{\prime}\right)}=\sum 1_{(1)} \otimes 1_{\left(1^{\prime}\right)} 1_{(2)} \otimes 1_{\left(2^{\prime}\right)} \\
\varepsilon(h k l)=\sum \varepsilon\left(h k_{(1)}\right) \varepsilon\left(k_{(2)} l\right)=\sum \varepsilon\left(h k_{(2)}\right) \varepsilon\left(k_{(1)} l\right)
\end{gathered}
$$

for all $h, k, l \in H$. Here $\Delta(1)=\sum 1_{(1)} \otimes 1_{(2)}=\sum 1_{\left(1^{\prime}\right)} \otimes 1_{\left(2^{\prime}\right)}$.

When considering such a structure it becomes natural to consider certain projections $H \rightarrow H$, since these maps will feature a lot in what what follows we label them as follows.

$$
\begin{aligned}
\Pi^{L}(h) & =\sum \varepsilon\left(_{(1)} h\right) 1_{(2)}, & \bar{\Pi}^{L}(h) & =\sum \varepsilon\left(_{(2)} h\right) 1_{(1)}, \\
\Pi^{R}(h) & =\sum \varepsilon\left(h 1_{(2)}\right) 1_{(1)}, & \bar{\Pi}^{R}(h) & =\sum \varepsilon\left(h 1_{(1)}\right) 1_{(2)} .
\end{aligned}
$$

Firstly using these projections one can give sensible conditions for an antipode. 
Definition 2.2.2. A weak Hopf algebra is a weak $k$-bialgebra with a $k$-linear map $S: H \rightarrow$ $H$, called the antipode, such that, for all $h \in H$,

$$
\sum h_{(1)} S\left(h_{(2)}\right)=\Pi^{L}(h), \quad \sum S\left(h_{(1)}\right) h_{(2)} S\left(h_{(3)}\right)=S(h), \quad \sum S\left(h_{(1)}\right) h_{(2)}=\Pi^{R}(h) .
$$

With this definition the antipode is both an anti-algebra and an anti-coalgebra map. Furthermore it satisfies

$$
\Pi^{L}=\bar{\Pi}^{R} \circ S, \quad \Pi^{R}=\bar{\Pi}^{L} \circ S,
$$

and

$$
S \circ \Pi^{L}=\Pi^{R} \circ S, \quad S \circ \Pi^{R}=\Pi^{L} \circ S .
$$

With mind to generalize Doi-Koppinen structures to the case where the auxiliary bialgebra is replaced with a weak bialgebra, notions of (co)module (co)algebras for weak bialgebras were developed in [2]. Such objects were defined as follows.

Definition 2.2.3. Given a weak bialgebra $H$, a right $H$-comodule algebra is a $k$-algebra $A$ with a right $H$ coaction $\rho^{A}: A \rightarrow A \otimes H$, such that for all $a, b \in A$,

$$
\rho^{A}(a b)=\rho^{A}(a) \rho^{A}(b),
$$

and

$$
\sum a_{[0]} \otimes \Pi^{L}\left(a_{[1]}\right)=\sum 1_{[0]} a \otimes 1_{[1]}
$$

Given the multiplicativity of $\rho^{A}(2.6)$, as was noticed in [2, Definition 2.1] and [15, Proposition 4.10], there are numerous equivalent ways in which to state the condition (2.7). We list these for future reference.

$$
\begin{array}{r}
\rho^{2}(1)=\sum 1_{[0]} \otimes 1_{[1]} 1_{(1)} \otimes 1_{(2)}, \\
\rho^{2}(1)=\sum 1_{[0]} \otimes 1_{(1)} 1_{[1]} \otimes 1_{(2)}, \\
\sum a_{[0]} \otimes \bar{\Pi}^{R}\left(a_{[1]}\right)=\sum a 1_{[0]} \otimes 1_{[1]}, \\
\sum 1_{[0]} \otimes \bar{\Pi}^{R}\left(1_{[1]}\right)=\rho(1) \\
\sum 1_{[0]} \otimes \Pi^{L}\left(1_{[1]}\right)=\rho(1) .
\end{array}
$$

Dually one considers the following

Definition 2.2.4. A right $H$-module coalgebra is defined as a coalgebra $C$ and a right $H$ module such that, for all $h, k \in H$ and $c \in C$,

$$
\Delta_{C}(c h)=\sum c_{(1)} h_{(1)} \otimes c_{(2)} h_{(2)}
$$

and

$$
\varepsilon_{C}(c h k)=\sum \varepsilon_{C}\left(h_{(2)}\right) \varepsilon_{H}\left(h_{(1)} k\right)
$$

Again with this dual definition given that $\Delta_{C}$ satisfies the comultiplicative condition 2.13 there are several equivalent statements which can replace 2.14 , see [15, Proposition 4.12] for details. Left $H$-comodule algebras and left $H$-module coalgebras can be defined in a similar way. 
Definition 2.2.5. A weak right-right Doi-Koppinen structure is a triple $(H, A, C)$ where $H$ is a weak $k$-bialgebra, $A$ a right $H$-comodule algebra and $C$ a right $H$-module coalgebra. The category of modules corresponding to such a triple, denoted $\overline{\mathbf{M}}(H)_{A}^{C}$, has objects which are right $C$-comodules with a right $A$-action satisfying the compatibility condition

$$
\rho^{M}(m a)=\sum m_{[0]} a_{[0]} \otimes m_{[1]} a_{[1]}
$$

and morphisms which are right $A$-linear and right $C$-colinear maps. Objects of this category are called weak Doi-Koppinen Hopf-modules.

Similarly one can define left-right, right-left and left-left weak Doi-Koppinen structures and their corresponding categories of modules, denoted ${ }_{A} \overline{\mathbf{M}}(H)^{C},{ }^{C} \overline{\mathbf{M}}(H)_{A}$ and ${ }_{A}^{C} \overline{\mathbf{M}}(H)$ respectively.

Now a reasonable question to ask is whether it is possible to relax the axioms of an entwining structure in order replace the auxiliary weak $k$-bialgebra in a weak right-right Doi-Koppinen structure, in a similar way to what had been done in the non-weak case. This question was resolved in [15] where a more general type of entwining was introduced as follows.

Definition 2.2.6. A triple $\left(A, C, \psi_{R}\right)$ consisting of a $k$-algebra $A, k$-coalgebra $C$ and $k$-linear map $\psi_{R}: C \otimes A \rightarrow A \otimes C$ is called a (right-right) weak entwining structure provided the map $\psi_{R}$ satisfies the conditions encoded in the following commutative diagram.

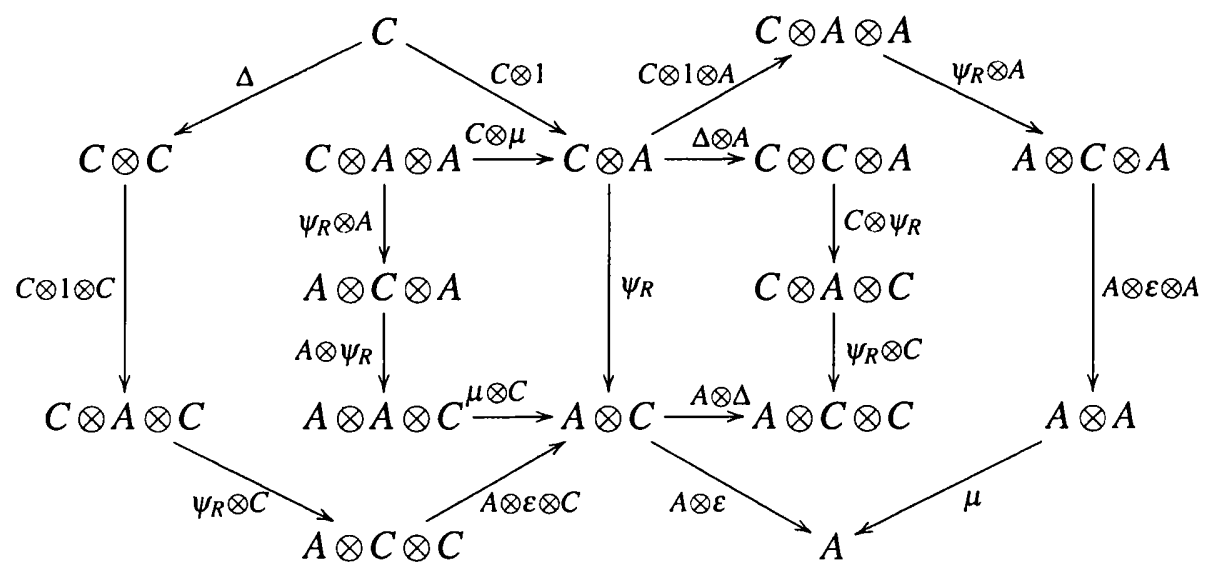

Writing, for all $a \in A$ and $c \in C, \psi_{R}(c \otimes a)=\sum_{\alpha} a_{\alpha} \otimes c^{\alpha}, \psi_{R}(c \otimes a)=\sum_{\beta} a_{\beta} \otimes c^{\beta}$ etc., these conditions can be expressed as

$$
\begin{array}{r}
\sum_{\alpha}(a b)_{\alpha} \otimes c^{\alpha}=\sum_{\alpha, \beta} a_{\alpha} b_{\beta} \otimes c^{\alpha \beta} \\
\sum_{\alpha} a_{\alpha} \varepsilon_{C}\left(c^{\alpha}\right)=\sum_{\alpha} \varepsilon_{C}\left(c^{\alpha}\right) 1_{\alpha} a \\
\sum_{\alpha} a_{\alpha} \otimes \Delta_{C}\left(c^{\alpha}\right)=\sum_{\alpha, \beta} a_{\alpha \beta} \otimes c_{(1)}^{\beta} \otimes c_{(2)}{ }^{\alpha} \\
\sum_{\alpha} 1_{\alpha} \otimes c^{\alpha}=\sum_{\alpha} \varepsilon_{C}\left(c_{(1)}^{\alpha}\right) 1_{\alpha} \otimes c_{(2)} .
\end{array}
$$


Modules over right-right weak entwining structures are defined with the same compatibility as in the non-weak case. We use the same notation for the category of comodules weak entwining structures, i.e. $\mathbf{M}(\psi)_{A}^{C}$. Also as in in the non-weak case these conditions are self dual, in the sense they are invariant under the operation which replaces $A$ with $C$, $\varepsilon_{C}$ with 1 and $\Delta$ with $\mu$.

Definition 2.2.7. A triple $(A, C, \psi)$ consisting of a $k$-algebra $A, k$-coalgebra $C$ and $k$-linear map $\psi_{L}: A \otimes C \rightarrow C \otimes A$ is called a (left-left) weak entwining structure provided the map $\psi$ satisfies the conditions encoded in the diagram obtained from the diagram in Definition 2.2.6, by reversing the $\psi_{R}$ arrows and replacing $\psi_{R}$ with $\psi_{L}$. Writing, for all $a \in A$ and $c \in C, \psi_{L}(a \otimes c)=\sum_{E} c_{E} \otimes a^{E}, \psi_{L}(a \otimes c)=\sum_{F} c_{F} \otimes a^{F}$ etc., these conditions can be expressed as

$$
\begin{array}{r}
\sum_{E} c_{E} \otimes(a b)^{E}=\sum_{E, F} c_{E F} \otimes a^{F} b^{E}, \\
\sum_{E} \varepsilon_{C}\left(c_{E}\right) a^{E}=\sum_{E} a \varepsilon_{C}\left(c_{E}\right) 1^{E}, \\
\sum_{E} \Delta_{C}\left(c_{E}\right) \otimes a^{E}=\sum_{E, F} c_{(1) E} \otimes c_{(2) F} \otimes a^{E F} \\
\sum_{E} c_{E} \otimes 1^{E}=\sum_{E} c_{(1)} \otimes \varepsilon_{C}\left(c_{(2) E}\right) 1^{E} .
\end{array}
$$

Modules over left-left weak entwining structures are defined with the same compatibility as in the non-weak case. Similarly one is able to define (left-right) and (right-left) weak entwining structures and their associated categories of modules.

Remark 2.2.8. Just as in the non-weak case for any weak right-right Doi-Koppinen structure $(H, A, C)$ one is able to give a corresponding right-right entwining structure $\left(A, C, \psi_{H}\right)$ where $\psi_{H}(c \otimes a):=\sum a_{[0]} \otimes c a_{[1]}$. Furthermore the modules associated to these corresponding structures are the same, i.e. $\overline{\mathbf{M}}_{A}^{C}(H)=\mathbf{M}\left(\psi_{H}\right)_{A}^{C}$.

\subsubsection{Generalizing results from non-weak case}

With the axioms for a weak entwining structure in place, we now investigate how it is possible to generalize some of the results for non-weak entwining structures to weak entwining structures. We start by showing how it is possible to associated an $A$-coring to a weak entwining structure. This result builds upon the first part of Proposition 2.1.6 and first appeared in [4, Proposition 2.3].

Proposition 2.2.9. Let $\left(A, C, \psi_{R}\right)$ be a right-right weak entwining structure. Define the map

$$
p_{R}: A \otimes C \rightarrow A \otimes C, \quad p_{R}:=(\mu \otimes C) \circ\left(C \otimes \psi_{R}\right) \circ(A \otimes C \otimes 1),
$$

and the set

$$
\mathfrak{C}:=\operatorname{Im} p_{R}=\left\{\sum_{i, \alpha} a_{i} 1_{\alpha} \otimes c_{i}^{\alpha} \mid \sum_{i} a_{i} \otimes c_{i} \in A \otimes C\right\} .
$$

Then $p_{R}$ is a projection, i.e. $p_{R} \circ p_{R}=p_{R}$, and 
(1) $\mathfrak{C}$ is an $(A, A)$-bimodule with the left action $a^{\prime}\left(\sum_{\alpha} a 1_{\alpha} \otimes c^{\alpha}\right)=\sum_{\alpha} a^{\prime} a 1_{\alpha} \otimes c^{\alpha}$ and the right action $\left(\sum_{\alpha} a^{\prime} 1_{\alpha} \otimes c^{\alpha}\right) a=\sum_{\alpha, \beta} a^{\prime} 1_{\alpha} a_{\beta} \otimes c^{\alpha \beta}=\sum_{\alpha} a^{\prime} a_{\alpha} \otimes c^{\alpha}$.

(2) $\mathfrak{C}$ is an $A$-coring with counit $\varepsilon_{\mathfrak{C}}=\left.\left(A \otimes \varepsilon_{C}\right)\right|_{\mathfrak{C}}$ and coproduct, for all $a \in A, c \in C$,

$$
\Delta_{\mathfrak{C}}\left(\sum_{\alpha} a 1_{\alpha} \otimes c^{\alpha}\right)=\sum_{\alpha} a 1_{\alpha} \otimes c^{\alpha}{ }_{(1)} \underset{A}{\otimes} \otimes c^{\alpha}{ }_{(2)}
$$

(3) $\mathbf{M}\left(\psi_{R}\right)_{A}^{C} \equiv \mathbf{M}^{\mathfrak{C}}$.

Similarly for a left-left weak entwining structure there is another version of this proposition.

Proposition 2.2.10. Let $\left(A, C, \psi_{L}\right)$ be a left-left weak entwining structure. Define the map

$$
p_{L}: C \otimes A \rightarrow C \otimes A, \quad p_{L}=(C \otimes \mu) \circ\left(\psi_{L} \otimes A\right) \circ(1 \otimes C \otimes A),
$$

and the set

$$
\mathfrak{D}:=\operatorname{Im} p_{L}=\left\{\sum_{i, E} c_{E}^{i} \otimes 1^{E} a^{i} \mid \sum_{i} c^{i} \otimes a^{i} \in C \otimes A\right\} .
$$

Then $p_{L}$ is a projection, i.e. $p_{L} \circ p_{L}=p_{L}$, and

(1) $\mathfrak{D}$ is an $(A, A)$-bimodule with the left action $a\left(\sum_{E} c_{E} \otimes 1^{E} a^{\prime}\right)=\sum_{E, F} c_{E F} \otimes a^{F} 1^{E} a^{\prime}$ and the right action $\left(\sum_{E} c_{E} \otimes 1^{E} a^{\prime}\right) a=\sum_{E} c_{E} \otimes 1^{E} a^{\prime} a$.

(2) $\mathfrak{D}$ is an $A$-coring with counit $\varepsilon_{\mathfrak{D}}=\left.\left(\varepsilon_{C} \otimes A\right)\right|_{\mathfrak{D}}$ and coproduct, for all $a \in A, c \in C$,

$$
\Delta_{\mathfrak{D}}\left(\sum_{E} c_{E} \otimes 1^{E} a\right)=\sum_{E} c_{E(1)} \otimes 1 \underset{A}{\otimes} c_{(2) E} \otimes 1^{E} a
$$

(3) ${ }_{A}^{C} \mathbf{M}\left(\psi_{L}\right) \equiv{ }^{\mathfrak{D}} \mathbf{M}$.

Recall we have already seen that it is possible to associate an entwining structure to a $C$-Galois extension $B \subseteq A$. Is there some more general type of extension to which it is possible to associate a weak entwining structure?

Definition 2.2.11. Let $C$ be a coalgebra and $A$ algebra that is also a right $C$-comodule. Then let

$$
B:=\left\{b \in A \mid \rho^{A}(b a)=b \rho^{A}(a), \forall a \in A\right\},
$$

denote the subalgebra of (right) coinvariants. We say that $B \subseteq A$ is a (right) weak coalgebraGalois extension (or weak $C$-Galois extension) provided that the left $A$-linear right $C$ colinear map

$$
\operatorname{can}_{A}: A \underset{B}{\otimes} A \rightarrow A \otimes C, \quad \operatorname{can}_{A}:=(\mu \otimes C) \circ\left(A \underset{B}{\otimes} \rho^{A}\right),
$$

is a split monomorphism in the category ${ }_{A} \mathbf{M}^{C}$, that is to say there exists some morphism $\chi: A \otimes C \rightarrow \underset{B}{\otimes} A$ in ${ }_{A} \mathbf{M}^{C}$ such that $\chi \circ \operatorname{can}_{A}=\underset{B}{A \otimes} A$.

Now for such an extension there is the following result 
Theorem 2.2.12. Let $B \subseteq A$ be a weak $C$-Galois extension. Then there exists a unique right-right weak entwining structure $\left(A, C, \psi_{R}\right)$ such that $A \in \mathbf{M}\left(\psi_{R}\right)_{A}^{C}$ with the canonical right $A$ action (multiplication in $A$ ) and the predetermined right $C$-coaction. This weak entwining structure is referred to as the canonical weak entwining structure associated to the weak $C$-Galois extension $B \subseteq A$.

Proof. Let $\chi$ be the map which splits $\operatorname{can}_{A}$ and set

$$
\tau: C \rightarrow \underset{B}{A \otimes A} A, \quad \tau:=\chi(1 \otimes c),
$$

to be what we will refer to as the weak translation map. Then the map

$$
\psi_{R}: C \otimes A \rightarrow A \otimes C, \quad \psi_{R}:=\operatorname{can}_{A} \circ(A \underset{B}{\otimes} \mu) \circ(\tau \otimes A),
$$

is the weak entwining map with the required properties. See [14, Subsection 37.9] for more details.

Proposition 2.2.13. Let $\mathfrak{C}$ be an $A$-coring associated to a right-right weak entwining structure, $\left(A, C, \psi_{R}\right)$, and $g \in \mathfrak{C}$ be a grouplike element. Then $\mathfrak{C}$ is a Galois $A$-coring with respect to $g$ if and only if $\operatorname{can}_{A}: A \otimes_{B} A \rightarrow C \otimes A$ is injective and $\mathfrak{C} \subset \operatorname{Im}\left(\operatorname{can}_{A}\right)$, where the right $C$ coaction on $A$ used in the computation of can $_{A}$ is that induced by $g$ via Proposition 2.1.9 and Theorem 2.2.9.

Proof. By Proposition 2.1.9 for the grouplike element $\mathrm{g}$, there is a corresponding right $\mathfrak{C}$-coaction on $A, \rho^{\mathfrak{C}}(a)=1 \underset{A}{\otimes} g a$, and in turn by Theorem 2.2 .9 a corresponding right $C$-coaction on $A, \rho^{A}=g a$, for which $A \in \mathbf{M}\left(\psi_{R}\right)_{A}^{C}$. Clearly with these choices of coactions

$$
A^{\mathrm{coC}}=\{b \in A \mid b g=g b\}=\left\{b \in A \mid \rho^{A}(b)=b \rho^{A}(1)\right\}=A^{\mathrm{coC}} .
$$

Therefore, setting $B:=A^{\mathrm{coC}}=A^{\mathrm{coC}}$ the unique $A$-coring map $\xi: A \underset{B}{\otimes} \rightarrow \mathfrak{C}$ determined by the condition $\xi(1 \underset{B}{\otimes} 1)=g$ (see Lemma 2.1.8) must be identically equal to $\operatorname{can}_{A}: A \underset{B}{\otimes} A \rightarrow$ $A \otimes C$, since

$$
\operatorname{can}_{A}\left(a \otimes a^{\prime}\right)=a \rho^{A}\left(a^{\prime}\right)=a g a^{\prime}=\xi\left(a \otimes a^{\prime}\right) .
$$

Hence the result follows.

Proposition 2.2.14. The A-coring associated to the weak entwining structure in Theorem 2.2.12 is a Galois A-coring. Conversely, if the A-coring associated to a weak entwining $\left(A, C, \psi_{R}\right)$ is a Galois A-coring, then $A$ is a weak $C$-Galois extension.

Proof. Suppose $\left(A, C, \psi_{R}\right)$ is as in Theorem 2.2.12 then $\operatorname{can}_{A}$ is clearly injective and since $A \in \mathbf{M}\left(\psi_{R}\right)_{A}^{C}, \rho^{C}(1) \in \mathfrak{C}$ is grouplike. Furthermore by the definition of $\psi_{R}$ it is clear that $\operatorname{Im}\left(\psi_{R}\right) \subseteq \operatorname{Im}\left(\operatorname{can}_{A}\right)$ and so since $\operatorname{can}_{A}$ is left $A$-linear all elements $a 1_{\alpha} \otimes c^{\alpha} \in$ $\operatorname{Im}\left(\operatorname{can}_{A}\right)$. Hence $\mathfrak{C} \subseteq \operatorname{Im}\left(\operatorname{can}_{A}\right)$ and $\mathfrak{C}$ is Galois with respect to $\rho^{C}(1) \in \mathfrak{C}$. Conversely, suppose $\mathfrak{C}$ is an $A$-coring associated to an entwining structure and that $\mathfrak{C}$ is Galois with respect to $g$. By the characterization of such a coring given in proposition 2.2.13 must have 
that $\mathfrak{C} \subseteq \operatorname{Im}\left(\operatorname{can}_{A}\right)$ and the map $c a n_{A}$ is injective. Also note that from the proof of 2.2 .13 it is clear that in fact $\operatorname{Im}\left(\operatorname{can}_{A}\right)=\mathfrak{C}$. Hence the corestriction $\overline{c a n}_{A}: A \otimes A \rightarrow \mathfrak{C}$ is invertible.

Since $\operatorname{can}_{A}$ is a morphism in ${ }_{A} \mathbf{M}^{C}$ it is clear that $\overline{c a n}_{A}^{-1}$ must also be a morphism in this category. Now observe that $p_{R}: A \otimes C \rightarrow \mathfrak{C}$ is also morphism in ${ }_{A} \mathbf{M}^{C}$. That it is left $A$-linear is clear. To see it is right $C$-colinear observe that

$$
\begin{aligned}
\rho^{\mathfrak{C}} \circ p_{R}(a \otimes c) & =\sum_{\alpha} a 1_{\alpha} \otimes c^{\alpha}{ }_{(1)} \otimes c^{\alpha}{ }_{(2)} \\
& =\sum_{\alpha} a 1_{\alpha \beta} \otimes c_{(1)}{ }^{\beta} \otimes c_{(2)}{ }^{\alpha} \\
& =\sum_{\alpha} a 1_{\alpha \beta} \varepsilon\left(c_{(2)}{ }^{\alpha}\right) \otimes c_{(1)}{ }^{\beta} \otimes c_{(3)} \\
& =\sum_{\alpha} a 1_{\alpha} \varepsilon\left(c_{(1)}{ }^{\alpha}{ }_{(2)}\right) \otimes c_{(1)}{ }^{\alpha}{ }_{(1)} \otimes c_{(2)} \\
& =\sum_{\alpha} a 1_{\alpha} \otimes c_{(1)}{ }^{\alpha} \otimes c_{(2)} .
\end{aligned}
$$

By use of relations (2.18) and (2.19). Having made these considerations it is clear that the composition $\overline{c a n}_{A}^{-1} \circ p_{R}: A \otimes C \rightarrow A \underset{B}{\otimes}$ splits $\operatorname{can}_{A}$ in the required way. Hence $A$.is a weak coalgebra-Galois extension.

\subsection{Invertible weak entwining structures}

With the notion of a weak-entwining structures in place, we now investigate what it should mean for a weak entwining structure to be invertible. We do this with mind to generalize results which require invertibility of an entwining structure in their assumptions in particular, [5, Theorem 4.6]. First observe this is a non-trivial problem since the obvious choice, requiring the entwining map to be bijective, leads to something unsatisfactory.

Proposition 2.3.1. If $\left(A, C, \psi_{R}\right)$ is a right-right weak entwining structure such that $\psi_{R}$ is bijective then $\left(A, C, \psi_{R}\right)$ must be a right-right entwining structure.

Proof. Let $\psi_{R}^{-1}: A \otimes C \rightarrow C \otimes A$ be the $k$-linear inverse of $\psi_{R}$, which must exist since $\psi_{R}$ is assumed to be bijective. Using the convention of writing $\psi_{R}^{-1}(a \otimes c)=\sum_{E} c_{E} \otimes a^{E}$ we immediately have that for all $a \in A$ and $c \in C$,

$$
\sum_{\alpha, E} c^{\alpha}{ }_{E} \otimes a_{\alpha}^{E}=c \otimes a, \quad \sum_{\alpha, E} a_{\alpha}^{E} \otimes c_{E}^{\alpha}=a \otimes c .
$$

Combining these with the relation (2.16) it is clear that, for all $a, b \in A$ and $c \in C$,

$$
\sum_{\alpha, E, F}\left(a^{F} b^{E}\right)_{\alpha} \otimes c_{E F}{ }^{\alpha}=\sum_{\alpha, E, F} a_{\alpha}^{F} b_{\beta}^{E} \otimes c_{E F}{ }^{\alpha \beta}=a b \otimes c .
$$

So applying $\psi_{R}^{-1}$ to the above we obtain, for all $a, b \in A$ and $c \in C$,

$$
\sum_{E, F} c_{E F} \otimes a^{F} b^{E}=\sum_{E} c_{E} \otimes(a b)^{E}
$$


With this knowledge, for any $a \in A$ and $c \in C$, we can perform the computation

$$
\begin{aligned}
\sum_{\alpha} a 1_{\alpha} \otimes c^{\alpha} & =\sum_{\alpha} \psi_{R} \circ \psi_{R}^{-1}\left(a 1_{\alpha} \otimes c^{\alpha}\right) \\
& =\sum_{\alpha} \psi_{R}\left(c^{\alpha}{ }_{E F} \otimes a^{F} 1_{\alpha}{ }^{E}\right) \\
& =\sum_{F} \psi_{R}\left(c_{F} \otimes a^{F}\right)=a \otimes c .
\end{aligned}
$$

Setting $a=1$ in the above it is clear that $\sum_{\alpha} 1_{\alpha} \otimes c^{\alpha}=1 \otimes c$. Furthermore this observation can be combined with relation (2.17) to compute that

$$
\sum_{\alpha} a_{\alpha} \varepsilon\left(c^{\alpha}\right)=\sum_{\alpha} \varepsilon\left(c^{\alpha}\right) a_{\alpha}=\varepsilon(c) a
$$

Hence $\left(A, C, \psi_{R}\right)$ is a right-right entwining structure.

Hence in the case of weak entwining structures a weaker notion of invertibility is required. We propose

Definition 2.3.2. An invertible weak entwining structure is a quadruple $\left(A, C, \psi_{R}, \psi_{L}\right)$ such that

(a) $\left(A, C, \psi_{R}\right)$ is a right-right weak entwining structure and $\left(A, C, \psi_{L}\right)$ is a left-left weak entwining structure;

(b) $\psi_{R} \circ \psi_{L}=p_{R}$ and $\psi_{L} \circ \psi_{R}=p_{L}$.

Initially when we proposed the definition in [13] we required a third condition, that for all $c \in C$

$$
\sum_{E} \varepsilon\left(c_{E}\right) 1^{E}=\sum_{\alpha} 1_{\alpha} \varepsilon\left(c^{\alpha}\right)
$$

However it was subsequently observed in [1] that in fact this is a consequence of the other assumptions. For completeness we recall the proof of this.

Proposition 2.3.3. For an invertible weak entwining structure $\left(A, C, \psi_{R}, \psi_{L}\right)$ equation (2.24) holds for all $c \in C$.

Proof. To see this equation is always satisfied take any $c \in C$ and compute

$$
\begin{aligned}
\sum_{\alpha, E} \varepsilon\left(c_{(1)}^{\alpha}\right) 1_{\alpha} \varepsilon\left(c_{(2) E}\right) 1^{E} & =\sum_{\alpha, E} 1_{\alpha} \varepsilon\left(c^{\alpha}{ }_{E}\right) 1^{E} \\
& =\sum_{\alpha, E} \varepsilon\left(c^{\alpha}{ }_{E}\right) 1_{\alpha}{ }^{E} \\
& =\sum_{E} \varepsilon\left(c_{E}\right) 1^{E}
\end{aligned}
$$

Where the first equality follows by relation (2.19), the second by (2.21) and the last equality since $\psi_{L} \circ \psi_{R}(c \otimes 1)=p_{L}(c \otimes 1)=\sum_{E} c_{E} \otimes 1^{E}$. However it is also possible to simplify the 
expression as

$$
\begin{aligned}
\sum_{\alpha, E} \varepsilon\left(c_{(1)}^{\alpha}\right) 1_{\alpha} \varepsilon\left(c_{(2) E}\right) 1^{E} & =\sum_{\alpha, E} \varepsilon\left(c_{E}^{\alpha}\right) 1_{\alpha} 1^{E} \\
& =\sum_{\alpha, E} \varepsilon\left(c_{E}^{\alpha}\right) 1_{\alpha}^{E} \\
& =\sum_{\alpha} \varepsilon\left(c^{\alpha}\right) 1_{\alpha}
\end{aligned}
$$

Where the first equality follows by relation (2.23), the second by (2.17) and the last equality since $\psi_{R} \circ \psi_{L}(1 \otimes c)=p_{R}(1 \otimes c)=\sum_{\alpha} 1_{\alpha} \otimes c^{\alpha}$.

As motivation for the definition of an invertible weak entwining structure the following observations were made. In the non-weak case the right-right entwining structure corresponding to a Doi-Koppinen structure $(H, A, C)$, where $H$ is a Hopf algebra with invertible antipode is invertible. To see this recall that the associated entwining map $\psi_{H}: C \otimes A \rightarrow A \otimes C$ is given by $\psi_{H}(c \otimes a)=\sum a_{[0]} \otimes c a_{[1]}$, with the knowledge that the antipode $S$ of $H$ is invertible it is possible to define an inverse for this map

$$
\psi_{H}^{-1}: A \otimes C \rightarrow C \otimes A, \quad a \otimes c \mapsto \sum c S^{-1} a_{[1]} \otimes a_{[0]}
$$

In the weak case one could expect the right-right entwining structure corresponding to a weak Doi-Koppinen structure $(H, A, C)$, where $H$ is a weak Hopf algebra with invertible antipode, to be invertible in a weaker sense. This is demonstrated in the following.

Proposition 2.3.4. Let $(H, A, C)$ be a right-right weak Doi-Koppinen structure such that $H$ is a weak Hopf algebra with an invertible antipode $S$. Then $\left(A, C, \psi_{R}, \psi_{L}\right)$ is an invertible entwining structure where $\psi_{R}$ is the associated right-right weak entwining map, i.e. $\psi_{R}(c \otimes$ $a)=\sum a_{[0]} \otimes c a_{[1]}$, and $\psi_{L}: A \otimes C \rightarrow C \otimes A$ is defined as $\psi_{L}(a \otimes c)=\sum c S^{-1}\left(a_{[1]}\right) \otimes a_{[0]}$.

Proof. We first need to establish that $\left(A, C, \psi_{L}\right)$ is a left-left weak entwining structure. Rather than checking the necessary relations directly we shall do this by showing that in fact $\psi_{L}$ is the natural left-left weak entwining structure associated to some left-left weak Doi-Koppinen structure $(\bar{H}, A, C)$. We define such as structure as follows. Firstly define $\bar{H}:=H^{o p}$, the opposite algebra to $H$. Agreeing to write $\bar{h}$ for element an element $h \in H$ but viewed in $\bar{H}$, there is a natural choice of left $\bar{H}$-module structure on $C$ given by

$$
\bar{h} c=c h,
$$

for all $c \in C$. Moreover this left $\bar{H}$-action is comultiplicative since the given right $H$-action on $C$ is comultiplicative and the comultiplication in $\bar{H}$ is the same as in $H$. Explicitly one computes

$$
\Delta_{C}(\bar{h} c)=\Delta_{C}(c h)=\sum c_{(1)} h_{(1)} \otimes c_{(2)} h_{(2)}=\sum \overline{h_{(1)}} c_{(1)} \otimes \overline{h_{(2)}} c_{(2)}=\sum \bar{h}_{(1)} c_{(1)} \otimes \bar{h}_{(2)} c_{(2)} .
$$

Also observe that, for all $c \in C$ and $k, h \in H$,

$$
\varepsilon_{C}(\bar{k} \bar{h} c)=\varepsilon_{C}(c h k)=\sum \varepsilon_{C}\left(c_{(2)}\right) \varepsilon_{H}\left(h_{(1)} k\right)=\sum \varepsilon_{H}\left(\bar{k} \bar{h}_{(1)}\right) \varepsilon_{C}\left(\bar{h}_{(2)} c\right)
$$


and so $C$ is a left $\bar{H}$-module coalgebra. Next as a candidate for a left $\bar{H}$ coaction on $A$ consider a $k$-linear map

$$
\lambda: A \rightarrow \bar{H} \otimes A, \quad a \mapsto \sum S^{-1} a_{[1]} \otimes a_{[0]} .
$$

That this defines a coaction follows immediately from the fact that $S$ and therefore $S^{-1}$ is an anti-coalgebra map. That $\lambda$ is a multiplicative map can easily be seen by using the multiplicativity of the right $H$-coaction on $A$ and the fact that $S^{-1}$ must be an anti-algebra map. Finally by virtue of equations (2.5) and (2.12) we can compute

$$
\sum \Pi^{R}\left(S^{-1} 1_{[1]}\right) \otimes 1_{[0]}=\sum S^{-1} \Pi^{L}\left(1_{[1]}\right) \otimes 1_{[0]}=\sum S^{-1} 1_{[1]} \otimes 1_{[0]},
$$

which can be seen as a left-handed version of condition 2.11. Hence $A$ is a left $\bar{H}$-comodule algebra and $(\bar{H}, A, C)$ is left-left weak Doi-Koppinen structure for which the associated entwining $\psi_{\bar{H}}$ must be equal to $\psi_{L}$ since

$$
\psi_{\bar{H}}(a \otimes c)=\sum a_{[-1]} c \otimes a_{[0]}=\sum c S^{-1} a_{[1]} \otimes a_{[0]}=\psi_{L}(a \otimes c) .
$$

Hence $\left(A, C, \psi_{L}\right)$ must be a left-left weak entwining structure.

To complete the proof we now show that $\psi_{R}$ and $\psi_{L}$ are compatible, in the sense of part (b) of Definition 2.3.2. First with these choices of entwining maps the corresponding projections can be written explicitly as

$$
p_{R}(a \otimes c)=\sum a 1_{[0]} \otimes c 1_{[1]}, \quad p_{L}(c \otimes a)=\sum c S^{-1} 1_{[1]} \otimes 1_{[0]} a .
$$

Now to verify the required compatibility first take any $a \in A$ and $c \in C$ and compute

$$
\begin{aligned}
\psi_{L} \circ \psi_{R}(c \otimes a) & =\psi_{L}\left(\sum a_{[0]} \otimes c a_{[1]}\right)=\sum c a_{[1](2)} S^{-1} a_{[1](1)} \otimes a_{[0]} \\
& =\sum c S^{-1}\left(a_{[1](1)} S a_{[1](2)}\right) \otimes a_{[0]}=\sum c S^{-1} \Pi^{L}\left(a_{[1]}\right) \otimes a_{[0]} \\
& =\sum c S^{-1} 1_{[1]} \otimes 1_{[0]} a=p_{L}(c \otimes a) .
\end{aligned}
$$

Where the third equality holds since $S$ and therefore $S^{-1}$ are anti-algebra maps. The fourth equality then follows by one of the defining characteristics of a weak Hopf algebra. The fifth equality follows since $A$ is a right $H$-comodule coalgebra and therefore condition (2.7) holds. It now only remains to show that the compositions of the entwining maps in the other order yields the required result. Before performing this calculation it will first be useful to note that, for all $h \in H$,

$$
S^{-1} \Pi^{R}(h)=\Pi^{L}\left(S^{-1} h\right)=\sum \varepsilon\left(h 1_{(1)}\right) 1_{(2)}=\bar{\Pi}^{R}(h) .
$$

This follows by the properties of the antipode stated in (2.4) and (2.5). Now take any $a \in A$ and $c \in C$ and compute

$$
\begin{aligned}
\psi_{R} \circ \psi_{L}(a \otimes c) & =\psi_{R}\left(\sum c S^{-1} a_{[1]} \otimes a_{[0]}\right)=\sum a_{[0]} \otimes c S^{-1}\left(a_{[1](2)}\right) a_{[1](1)} \\
& =\sum a_{[0]} \otimes c S^{-1}\left(\left(S a_{[1](1)}\right) a_{[1](2)}\right)=\sum a_{[0]} \otimes c S^{-1} \Pi^{R}\left(a_{[1]}\right) \\
& =\sum a_{[0]} \otimes c \bar{\Pi}^{R}\left(a_{[1]}\right)=\sum a 1_{[0]} \otimes c 1_{[1]}=p_{R}(a \otimes c) .
\end{aligned}
$$


Again the third equality holds since $S$ and therefore $S^{-1}$ are anti-algebra maps and the fourth equality then follows by one of the defining characteristics of a weak Hopf algebra. The fifth equality follows by the computation (2.26). Finally the sixth equality follows because $A$ is a right $H$-comodule algebra and therefore (2.10) holds. Therefore $\left(A, C, \psi_{R}, \psi_{L}\right)$ must be an invertible weak entwining structure.

In particular restricting this to the case of weak Doi Koppinen structures of the form $(H, A, H)$ we obtain

Corollary 2.3.5. Let $H$ be a weak Hopf algebra with an invertible antipode $S$ and let $A$ be a right $H$-comodule algebra. Then $\left(A, H, \psi_{R}, \psi_{L}\right)$ is an invertible entwining structure where $\psi_{R}$ is the associated right-right weak entwining map, i.e. $\psi_{R}(h \otimes a)=\sum a_{[0]} \otimes h a_{[1]}$, and

$$
\psi_{L}: A \otimes H \rightarrow H \otimes A, \quad a \otimes h \mapsto \sum h S^{-1} a_{[1]} \otimes a_{[0]}
$$

Before continuing it will be useful to point out an obvious consequence of Definition 2.3.2.

Lemma 2.3.6. Let $\left(A, C, \psi_{R}, \psi_{L}\right)$ be an invertible weak entwining structure. Then:

(1) $\psi_{R} \circ p_{L}=\psi_{R}$,

(2) $\psi_{L} \circ p_{R}=\psi_{L}$.

Proof. For (1) first observe

$$
p_{R} \circ \psi_{R}(c \otimes a)=\sum_{\alpha, \beta} a_{\alpha} 1_{\alpha} \otimes c^{\alpha \beta}=\sum_{\alpha} a_{\alpha} \otimes c^{\alpha},
$$

by the use of relation (2.16). So by the conditions (b) of definition 2.3 .2

$$
\psi_{R} \circ p_{L}=\psi_{R} \circ \psi_{L} \circ \psi_{R}=p_{R} \circ \psi_{R}=\psi_{R} .
$$

Part (2) of the this Lemma can be shown in a similar way.

We now investigate what an invertible weak entwining structure means for the $A$ corings which can be associated to its constituent weak entwining maps.

Proposition 2.3.7. Let $\left(A, C, \psi_{R}, \psi_{L}\right)$ be an invertible weak entwining structure and let $\mathfrak{C}=\operatorname{Im} p_{R}$ and $\mathfrak{D}=\operatorname{Im} p_{L}$ be the corresponding A-corings. Then the restrictions of the entwining maps

$$
\psi_{L}: \mathfrak{C} \rightarrow \mathfrak{D}, \quad \psi_{R}: \mathfrak{D} \rightarrow \mathfrak{C}
$$

are inverse isomorphisms of A-corings.

Proof. First observe that it does make sense to restrict the targets of $\psi_{R}$ and $\psi_{L}$ in this way since from the conditions (b) of Definition 2.3.2 it is apparent that $\operatorname{Im} \psi_{R} \subseteq \mathfrak{C}$ and $\operatorname{Im} \psi_{L} \subseteq \mathfrak{D}$. Moreover, since the maps $p_{R}$ and $p_{L}$ are projections these conditions immediately imply that the given restrictions of $\psi_{R}$ and $\psi_{L}$ are inverse isomorphisms of $k$-modules. Now we demonstrate that $\psi_{R}$ is a morphism of $A$-corings, starting by showing that it is $(A, A)$-bilinear. Firstly observe that, by Lemma 2.3.6, for all $a \in A$ and $c \in C$,

$$
\psi_{R}\left(\sum_{E} c_{E} \otimes 1^{E} a\right)=\psi_{R} \circ p_{L}(c \otimes a)=\psi_{R}(c \otimes a)=\sum_{\alpha} a_{\alpha} \otimes c^{\alpha} .
$$


We can also compute that

$$
\psi_{R}\left(\sum_{E} c_{E} \otimes 1^{E}\right) a=\sum_{\alpha}\left(1_{\alpha} \otimes c^{\alpha}\right) a=\sum_{\alpha, \beta} 1_{\alpha} a_{\beta} \otimes c^{\alpha \beta}=\sum_{\alpha} a_{\alpha} \otimes c^{\alpha}
$$

by using relation (2.16) in the last step. Therefore we conclude that $\psi_{R}$ is a right $A$-module map. We can also compute that, for all $a \in A$ and $c \in C$,

$$
\psi_{R}\left(\sum_{E} a\left(c_{E} \otimes 1^{E}\right)\right)=\psi_{R}\left(\sum_{E, F} c_{E F} \otimes a^{F} 1^{E}\right)=\psi_{R}\left(\sum_{E} c_{E} \otimes a^{E}\right)=p_{R}(a \otimes c)=\sum_{\alpha} a 1_{\alpha} \otimes c^{\alpha}
$$

where the second equality follows be the relation (2.20) and the third equality by the conditions (b) of Definition 2.3.2. Also using these conditions we can compute

$$
a \psi_{R}\left(\sum_{E} c_{E} \otimes 1^{E}\right)=a p_{R}(1 \otimes c)=\sum_{\alpha} a 1_{\alpha} \otimes c^{\alpha} .
$$

Therefore, since we have shown that $\psi_{R}$ is right $A$-linear, we conclude that $\psi_{R}$ is a left $A$-module map. This completes the proof that $\psi_{R}$ is an $(A, A)$-bimodule map.

Next we aim to show that $\psi_{R}$ respects the counit. To do this take any $c \in C$ and $a \in A$ and make the computation

$$
\begin{aligned}
\varepsilon_{\mathfrak{C}}\left(\psi_{R}\left(\sum_{E} c_{E} \otimes 1^{E} a\right)\right) & =\sum_{\alpha} a_{\alpha} \varepsilon_{C}\left(c^{\alpha}\right)=\sum_{\alpha} 1_{\alpha} \varepsilon_{C}\left(c^{\alpha}\right) a \\
& =\sum_{E} \varepsilon_{C}\left(c_{E}\right) 1^{E} a=\varepsilon_{\mathfrak{D}}\left(\sum_{E} c_{E} \otimes 1^{E} a\right) .
\end{aligned}
$$

Where the first equality holds by Lemma 2.3 .6 and the definition of $\varepsilon_{\mathfrak{C}}$. The second equality then follows by the relation (2.17) and the third by Proposition 2.3.3. The final equality follows immediately from the definition of $\varepsilon_{\mathfrak{D}}$. Hence $\psi_{R}$ is a counital map.

Finally we show that $\psi_{R}$ is comultiplicative. Again start by taking $c \in C$ and $a \in A$ and first computing that

$$
\Delta_{\mathfrak{C}} \circ \psi_{R}\left(\sum_{E} c_{E} \otimes 1^{E} a\right)=\Delta_{\mathfrak{C}}\left(\sum_{\alpha} a_{\alpha} \otimes c^{\alpha}\right)=\sum_{\alpha} a_{\alpha} \otimes c^{\alpha}{ }_{(1)} \otimes c^{\alpha}{ }_{(2)},
$$

by using Lemma 2.3.6 and the definition of coproduct in $\mathfrak{C}$. On the other hand,

$$
\begin{aligned}
\left(\psi_{R} \otimes \psi_{R}\right) \circ \Delta_{\mathfrak{D}}\left(\sum_{E} c_{E} \otimes 1^{E} a\right) & =\sum_{E} \psi_{R}\left(c_{E(1)} \otimes 1\right) \underset{A}{\otimes} \psi_{R}\left(c_{E(2)} \otimes 1^{E} a\right) \\
& =\sum_{E, F} \psi_{R}\left(c_{(1) E} \otimes 1\right) \underset{A}{\otimes} \psi_{R}\left(c_{(2) F} \otimes 1^{E F} a\right) \\
& =\sum_{E} \psi_{R}\left(c_{(1) E} \otimes 1^{E}\right) \underset{A}{\otimes} \psi_{R}\left(c_{(2)} \otimes a\right) \\
& =\sum_{\alpha, \beta} 1_{\alpha} \otimes c_{(1)}^{\alpha} \underset{A}{\otimes} a_{\beta} \otimes c_{(2)}^{\beta} \\
& =\sum_{\alpha, \beta, \gamma} 1_{\alpha} a_{\beta \gamma} \otimes c_{(1)} \underset{A}{\alpha \gamma} \underset{A}{1} \otimes c_{(2)} \beta \\
& =\sum_{\alpha} a_{\alpha} \otimes c_{(1)}^{\alpha}{ }_{A} \underset{A}{1} \otimes c_{(2)}^{\alpha}=\Delta_{\mathfrak{C}}\left(\sum_{\alpha} a_{\alpha} \otimes c^{\alpha}\right) .
\end{aligned}
$$


Where the first equality follows by the definition of the coproduct in $\mathfrak{C}$ and the second by the relation (2.22). The third equality follows by the bimodule structure of $\mathfrak{D}$ and the fact that $\psi_{R}$ is an $(A, A)$-bimodule map. Next part (b) of Definition 2.3.2 is applied, noting that $\sum p_{R}\left(1 \otimes c_{(1)}\right) \otimes c_{(2)}=\sum_{\alpha} 1_{\alpha} \otimes c_{(1)}{ }^{\alpha} \otimes c_{(2)}$. The fifth and sixth equalities follow by the applications of relations (2.16) and (2.18). The final equality is just the definition of the comultiplication in $\mathfrak{C}$. Thus $\psi_{R}$ is a morphism of $A$-corings. Similarly one can demonstrate that $\psi_{L}$ is also an $A$-coring map.

In the case of invertible entwining structures it was shown in [7, Section 6] that if $A \in$ $\mathbf{M}(\psi)_{A}^{C}$ then $A \in{ }_{A}^{C} \mathbf{M}\left(\psi^{-1}\right)$. Similarly in the weak case we can use the previous proposition to show

Corollary 2.3.8. Let $\left(A, C, \psi_{R}, \psi_{L}\right)$ be an invertible weak entwining structure. If $A \in$ $\mathbf{M}\left(\psi_{R}\right)_{A}^{C}$, then $A \in{ }_{A}^{C} \mathbf{M}\left(\psi_{L}\right)$ with the coaction

$$
{ }^{A} \rho(a)=\psi_{L}\left(\sum a 1_{[0]} \otimes 1_{[1]}\right) .
$$

Proof. Suppose that $A \in \mathbf{M}\left(\psi_{R}\right)_{A}^{C}$, then, by the correspondence given in Theorem 2.2 .9 , it is clear that $A$ is right $\mathfrak{C}$-comodule. Therefore, by Proposition 2.1 .9 , there is a corresponding grouplike element $g \in \mathfrak{C}$ where $g=\rho^{A}(1)=\sum 1_{[0]} \otimes 1_{[1]}$. Since one can just as easily show a left-handed version of Proposition 2.1.9, $A$ is a left $\mathfrak{C}$-comodule with the coaction $a \mapsto a g \otimes 1$. Now by Proposition 2.3.7, the map $\psi_{L}: \mathfrak{C} \rightarrow \mathfrak{D}$ is an isomorphism of $A$-corings. Therefore using $\psi_{L}$ it is possible to give a left $\mathfrak{D}$-coaction on $A$, explicitly

$$
{ }^{A} \rho(a)=\psi_{L}(a g) \underset{A}{\otimes 1}
$$

Therefore $A \in{ }_{A}^{C} \mathbf{M}\left(\psi_{L}\right)$ with the coaction ${ }^{A} \rho(a)=\psi_{L}\left(\sum a 1_{[0]} \otimes 1_{[1]}\right)$, as stated. 


\section{Chapter 3}

\section{Galois theory of $C$-rings}

\subsection{Introduction}

In this chapter we develop the theory of objects which are dual to $A$-corings which were first defined in [4, Section 6]. In order to avoid making any flatness assumptions in this chapter all algebras, coalgebras etc. will be over a field $k$. Recall

Definition 3.1.1. A $C$-ring is a $(C, C)$-bicomodule $\mathscr{A}$ together with two $(C, C)$-bicomodule maps $\eta_{\mathscr{A}}: C \rightarrow \mathscr{A}$ and $\mu_{\mathscr{A}}: \mathscr{A} \square_{C} \mathscr{A} \rightarrow \mathscr{A}$ which render the diagrams
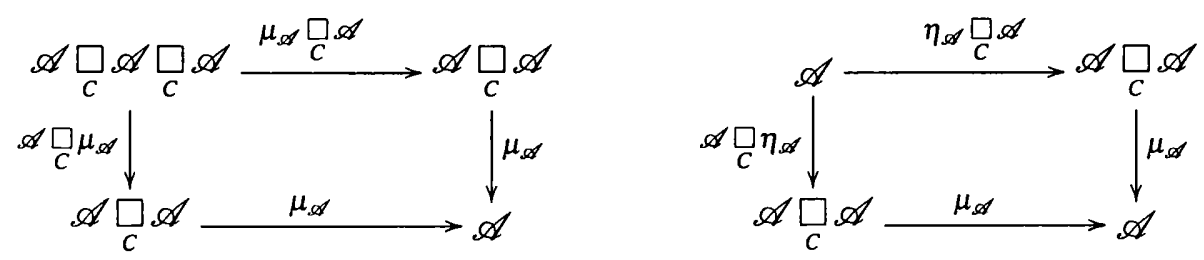

commutative, where the standard isomorphisms $\mathscr{A} \square_{C} C \simeq \mathscr{A} \simeq C \square_{C} \mathscr{A}$ provided by the $C$ coactions are implicit. The map $\eta_{\mathscr{A}}$ is referred to as the unit and map $\mu_{\mathscr{A}}$ as the product. A morphism of $C$-rings is a map $f: \mathscr{A} \rightarrow \mathscr{B}$ satisfying

$$
\mu_{\mathscr{B}} \circ\left(f \square_{C} f\right)=f \circ \mu_{A}, \quad f \circ \eta_{\mathscr{A}}=\eta_{\mathscr{B}} .
$$

In principle the theory of such objects should be just as rich as the theory of corings. Importantly, where as $A$-corings can be used to study algebra extensions, we can expect to study coalgebra coextensions using $C$-rings.

Example 3.1.2. To any coalgebra map $f: C \rightarrow D$ it is possible to associate a $C$-ring as follows. First define $\mathscr{A}=C \square_{D} C$ viewing $C$ as a $(D, D)$-bicomodule via the map $f$ and let $\mathscr{A}$ have the natural choice of $(C, C)$-bicomodule structure. Then set

$$
\mu_{\mathscr{A}}: \mathscr{A} \square_{C} \mathscr{A} \rightarrow \mathscr{A}, \quad c^{1} \otimes c^{2} \otimes c^{3} \otimes c^{4} \mapsto c^{1} \varepsilon\left(c^{2}\right) \otimes \varepsilon\left(c^{3}\right) c^{4}
$$

and

$$
\eta_{\mathscr{A}}: C \rightarrow \mathscr{A}, \quad c \mapsto \sum c_{(1)} \otimes c_{(2)}
$$

We shall refer to a $C$-ring arising in this way as a $S$ weedler $C$-ring. 
It is clear that $C$-rings generalize algebras and that a notion of a module for a algebra can be extended in a sensible way to $C$-rings as

Definition 3.1.3. A (right) module for a $C$-ring is a pair $\left(M, \overline{\rho_{M}}\right)$ where $M$ is a right $C$ comodule and $\overline{\rho_{M}}: M \square_{C} \mathscr{A} \rightarrow M$ is a map of right $C$-comodules satisfying

$$
\overline{\rho_{M}} \circ\left(\overline{\rho_{M}} \square_{C} \mathscr{A}\right)=\overline{\rho_{M}} \circ\left(M \square_{C} \mu_{\mathscr{A}}\right), \quad \overline{\rho_{M}} \circ\left(M \square_{C} \eta_{\mathscr{A}}\right)=M .
$$

Where again the standard isomorphism $M \square C C \simeq M$ provided by the $C$-coaction on $M$ is implicit in what is written. The map $\overline{\rho_{M}}$ is referred to as a right $\mathscr{A}$-action and just as for standard modules, the above conditions are referred to as associativity and unitality. The category of all such objects with morphisms $f: M \rightarrow N$ which are right $C$-colinear and right $\mathscr{A}$-linear maps (in the sense that $f \circ \overline{\rho_{M}}=\overline{\rho_{N}} \circ\left(f \square \square_{C}\right)$ ) is denoted by $\mathbf{M}_{\mathscr{A}}$.

\subsection{Matrix rings contexts}

In trying to further develop the theory of $C$-rings in the same way as has been outlined for corings one soon encounters a problem. In the case of $A$-corings, Galois comodules are assumed to be finitely generated and projective as $A$-modules, which is equivalent to saying that there is a finite dual basis. It is then standard to use such a basis in proving things about Galois comodules. However the notion of the existence of finite dual basis is not easily dualized (cf. Section 1.3). Instead we proceed by dualising the notion of a comatrix coring context as defined in [8].

\subsubsection{Quasi-finite matrix ring contexts}

Definition 3.2.1. A matrix ring context, $\left(C, D,{ }^{C} N^{D},{ }^{D} M^{C}, \sigma, \tau\right)$, consists of a pair of coalgebras $C$ and $D$, a $(C, D)$-bicomodule $N$, a $(D, C)$-bicomodule $M$ and a pair of bicomodule maps

$$
\sigma: C \rightarrow N \underset{D}{\square} M, \quad \tau: M \square_{C} N \rightarrow D
$$

such that the diagrams
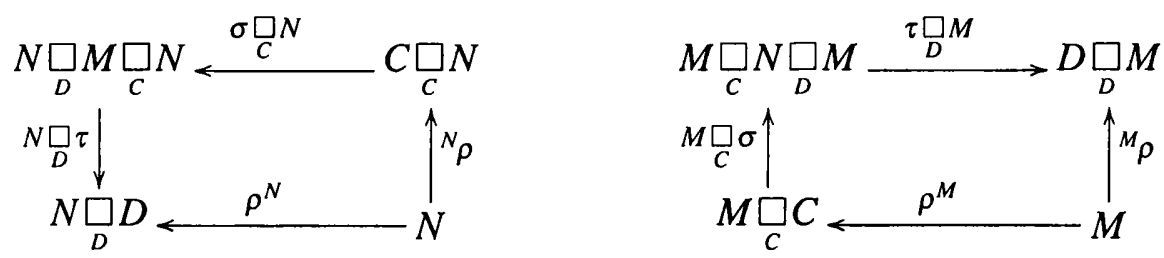

commute. The map $\sigma$ is called a unit and $\tau$ is called a counit of a matrix context.

By the assumption that the counit $\tau$ of a matrix ring context is $D$-bicolinear, it is uniquely determined by its reduced form $\widehat{\tau}=\varepsilon_{D} \circ \tau$. To see this not that any $D$-bicolinear map $f: L \rightarrow D$ can expressed in terms of its reduced form $\tilde{f}=\varepsilon_{D} \circ f$ as $f=(D \otimes \tilde{f}) \circ{ }^{L} \rho=$ $(\tilde{f} \otimes D) \circ \rho^{L}$. From this observation it is also clear that

$$
(D \otimes \widehat{\tau}) \circ\left({ }^{M} \rho \otimes N\right)=(\widehat{\tau} \otimes D) \circ\left(M \otimes \rho^{N}\right) .
$$


We shall refer to the map $\widehat{\tau}$ as the reduced counit of the matrix ring context. Using the counitality of the coactions on $M$ and $N$, the diagrams in Definition (3.2.1) can be rewritten in term of $\widehat{\tau}$ as

$$
(N \otimes \widehat{\tau}) \circ\left(\sigma \square_{C} N\right) \circ{ }^{N} \rho=N, \quad(\widehat{\tau} \otimes M) \circ\left(M \square_{C} \sigma\right) \circ \rho^{M}=M .
$$

As justification of the word context in our terminology recall the definition of pre-equivalence data or a Morita-Takeuchi context as was introduced in [38, Definition 2.3]. If in a context of this type

$$
\left(C, D,{ }^{C} N^{D},{ }^{D} M^{C}, f: C \rightarrow N \underset{D}{\square} M, g: D \rightarrow M \underset{C}{\square} N\right),
$$

the map $g$ is injective then by [38, Theorem 2.3] $g$ must be an isomorphism. Due to the constraints which are imposed on $f$ and $g$ in the definition of a Morita-Takeuchi context it is then apparent that

$$
\left(C, D,{ }^{C} N^{D},{ }^{D} M^{C}, f: C \rightarrow N \underset{D}{\square} M, g^{-1}: M \square_{C} N \rightarrow D\right),
$$

is a matrix ring context. Of greater importance to us, is the reason why the word ring appears in Definition 3.2.1

Proposition 3.2.2. Let $\left(C, D,{ }^{C} N^{D},{ }^{D} M^{C}, \sigma, \tau\right)$ be a matrix ring context. Then $\mathscr{A}:=N \square_{D} M$ is a $C$-ring with the product and unit

$$
\mu_{\mathscr{A}}=N \square_{D} \widehat{\tau} \square_{D} M, \quad \eta_{\mathscr{A}}=\sigma
$$

where $\widehat{\tau}$ is the reduced counit. Furthermore, $M$ is a right $\mathscr{A}$-module with the action $\widehat{\tau} \square_{D} M$ and $N$ is a left $\mathscr{A}$-module with the action $N \square \widehat{\tau}$. The $C$-ring $\mathscr{A}$ is called a matrix $C$-ring.

Proof. It is clear from their definitions that both $\mu_{\mathscr{A}}$ and $\eta_{\mathscr{A}}$ are $C$-bicolinear. To see that the map $\mu_{\mathscr{A}}$ is well defined, i.e. $\operatorname{Im}\left(\mu_{\mathscr{A}}\right) \subseteq N \square_{D} M$, observe that $\tau$ is $D$-bicolinear so $\operatorname{Im}\left(N \underset{D}{\square} \tau \square \square_{D} M\right) \subseteq N \square \square_{D} D \square_{D} M$. Similarly $N \square_{D} \varepsilon_{D}: N \square_{D} D \rightarrow N$ is right $D$-colinear so $\operatorname{Im}\left(N \square_{D} \varepsilon_{D} \square M\right) \subseteq N \square_{D} M$ and therefore

$$
\operatorname{Im}\left(\mu_{\mathscr{A}}\right)=\operatorname{Im}\left(\left(N \square_{D} \varepsilon_{D} \square_{D} M\right) \circ\left(N \square_{D} \tau \square \square_{D} M\right)\right) \subseteq N \square_{D} M .
$$

That $\mu_{\mathscr{A}}$ is associative is also easily verified as

$$
\begin{aligned}
& \left(N \underset{D}{\square} \widehat{\tau} \square_{D} M\right) \circ\left(N \square_{D} M \square_{C} N \square_{D} \widehat{\tau} \square_{D} M\right)=\left(N \underset{D}{\square} \widehat{\tau} \square_{D} \widehat{\tau} \square_{D} M\right) \\
& =(N \underset{D}{\square} \widehat{\tau} \underset{D}{\square} M) \circ\left(N \underset{D}{\widehat{\tau}} \square_{D} M \square_{C} N \square_{D} M\right) .
\end{aligned}
$$

Using equation (3.2) it is a straight forward calculation to show that $\eta_{\mathscr{A}}$ is the unit for $\mu_{\mathscr{A}}$. The statements about the actions of $\mathscr{A}$ are proven in a similar way.

Example 3.2.3. To any coalgebra coextension $f: C \rightarrow D$ it possible to associate a matrix ring context $\left(C, D,{ }^{C} C^{D},{ }^{D} C^{C}, \Delta_{C}, f\right)$, where the $D$-coactions on $C$ are those induced by $f$. The corresponding matrix $C$-ring is precisely the Sweedler type $C$-ring associated to the coextension. 
We now explore the meaning of a matrix ring context.

Proposition 3.2.4. If $\left(C, D,{ }^{C} N^{D},{ }^{D} M^{C}, \sigma, \tau\right)$ is a matrix ring context, then the cotensor functor $F=-\square_{C} N: \mathbf{M}^{C} \rightarrow$ Vect $_{k}$ is a left adjoint of the tensor functor $G=-\otimes M:$ Vect $_{k} \rightarrow$ $\mathbf{M}^{C}$.

Proof. Define natural transformations

$$
\begin{gathered}
\varphi: \mathbf{M}^{C} \rightarrow G F, \quad \varphi_{X}:=(X \underset{c}{\square} \sigma) \circ \rho^{X}, \\
v: F G \rightarrow \operatorname{Vect}_{k}, \quad v_{Y}:=\left(Y \otimes \varepsilon_{D}\right) \circ(Y \otimes \tau)=Y \otimes \widehat{\tau} .
\end{gathered}
$$

We need to show that these morphisms are the unit and counit, respectively, of the adjunction. Take any right $C$-comodule $X$ and compute

$$
\begin{aligned}
v_{F(X)} \circ F\left(\varphi_{X}\right) & =(X \underset{C}{\square} N \otimes \widehat{\tau}) \circ\left(\left((X \underset{C}{\square} \sigma) \circ \rho^{X}\right) \square_{C} N\right) \\
& =(X \underset{C}{\square} N \otimes \widehat{\tau}) \circ(X \underset{C}{\square} \underset{C}{\square} N) \circ\left(X \square_{C}^{N} \rho\right)=X \square_{C} N=F(X),
\end{aligned}
$$

where the second equality follows by the definition of the cotensor product, and the third equality follows by the first of equations (3.2). On the other hand, for all vector spaces $Y$,

$$
G\left(v_{Y}\right) \circ \varphi_{G(Y)}=(Y \otimes \widehat{\tau} \otimes M) \circ\left(Y \otimes M \square_{C} \sigma\right) \circ\left(Y \otimes \rho^{M}\right)=Y \otimes M=G(Y),
$$

by the second of equations (3.2). Hence the natural transformations $\varphi$ and $v$ satisfy the required properties.

Remark 3.2.5. Suppose $\left(C, D,{ }^{C} N^{D},{ }^{D} M^{C}, \sigma, \tau\right)$ is a matrix ring context. In view of section 1.3 and the previous proposition we can draw the following conclusions. Firstly since $-\otimes M:$ Vect $_{k} \rightarrow \mathbf{M}^{C}$ has a left adjoint, $M$ is a quasi-finite right $C$-comodule. Moreover by the uniqueness of adjoints there must be a functorial isomorphism $h_{C}(M,-) \simeq-\square_{C} N$. Now observe that $h_{C}(M,-)$ has a right adjoint so is right exact, and since $-\square_{C} N$ is left exact, the above isomorphism of functors implies that the co-hom functor must be exact. Therefore by Proposition 1.3.16 $M$ is an injector as a right $C$-comodule. Secondly, with the knowledge that $M$ is a quasi-finite injector as a right $C$-comodule, it then follows from Proposition 1.3.18 that there is a functorial isomorphism $h_{C}(M,-) \simeq-\square_{C} h_{C}(M, C)$. Combining this with the functorial isomorphism $h_{C}(M,-) \simeq-\square_{C} N$ it is then clear there must be an isomorphism $h_{C}(M, C) \simeq N$.

Theorem 3.2.6. Let $M$ be a $(D, C)$-bicomodule and suppose that the right $C$-comodule $M$ is a quasi-finite injector. Define $N:=h_{C}(M, C)$. Then there exist maps $\sigma$ and $\tau$ such that the sextuple $\left(C, D,{ }^{C} N^{D}, M^{C}, \sigma, \tau\right)$ is a matrix ring context.

First observe from Proposition 1.3.14 that the left $D$-coaction on $M$ must be induced from the natural left coaction of the coendomorphism coalgebra $E=h_{C}(M, M)$ by some unique choice of coalgebra map $\pi: E \rightarrow D$. With this in mind we will prove Theorem 3.2.6 by firstly considering the case where $D=E$. 
Lemma 3.2.7. Suppose that a right $C$-comodule $M$ is a quasi-finite injector and define $N:=h_{C}(M, C)$ and $E:=h_{C}(M, M)$. Then there exist maps $\sigma_{E}$ and $\tau_{E}$ such that the sextuple $\left(C, E,{ }^{C} N^{E},{ }^{E} M^{C}, \sigma_{E}, \tau_{E}\right)$ is a matrix ring context, where the coactions on $N$ and $M$ are as described in Propositions 1.3 .10 and 1.3.14 respectively.

Proof. Since $M$ is an $(E, C)$-bicomodule which is quasi-finite as a right $C$-comodule, by Theorem 1.3.12 the functor $-\square_{E} M: \mathbf{M}^{E} \rightarrow \mathbf{M}^{C}$ has a left adjoint, which is given by the co-hom functor viewed as a functor $h_{C}: \mathbf{M}^{C} \rightarrow \mathbf{M}^{E}$. Furthermore since $M^{C}$ is a quasi-finite injector, by Proposition 1.3.18, $h_{C}(M,-) \simeq-\square_{C} h_{C}(M, C)$. Therefore $\left(-\square_{C} N,-\square_{E} M\right)$ is an adjoint pair of functors. This is characterized by the existence of a unit and counit of adjunction,

$$
\varphi: \mathbf{M}^{C} \rightarrow-\underset{c}{\square} N \underset{E}{\square} M, \quad v:-\underset{E}{\square} M \underset{c}{\square} N \rightarrow \mathbf{M}^{E} .
$$

Using these maps define right comodule maps

$$
\sigma_{E}:=\varphi_{C}: C \rightarrow C \underset{C}{\square} N \square_{E} M \simeq N \underset{E}{\square} M, \quad \tau_{E}:=v_{E}: M \underset{C}{\square} N \simeq E \square_{E} M \square_{C} N \rightarrow E .
$$

In addition to being right comodule maps it turns out these maps are bimodule maps. To see that $\sigma_{E}$ is a $C$-bicomodule map use the fact that $\varphi$ is a natural transformation to produce commutative diagrams
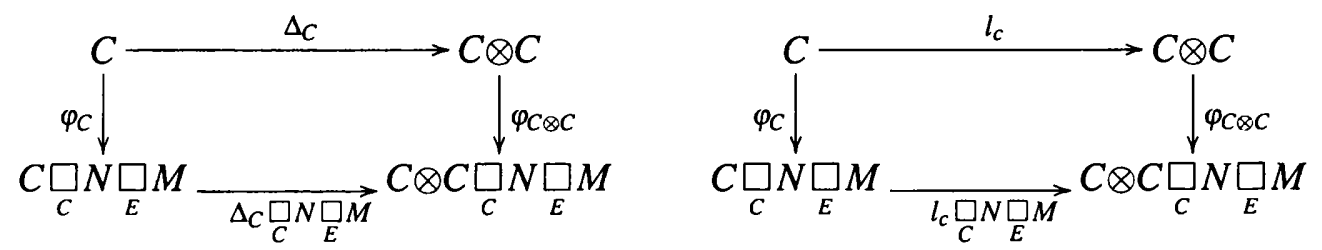

where $l_{c}\left(c^{\prime}\right)=c \otimes c^{\prime}$, for all $c, c^{\prime} \in C$. Since $\Delta_{C} \underset{c}{\square} N \underset{E}{\square} M$ can be identified with the left $C$-coaction ${ }_{E}^{N \square_{E} M} \rho$, putting these two diagrams together we obtain, for all $c \in C$,

$$
\begin{aligned}
& N \underset{E}{N{ }^{\prime} M} \rho \circ \varphi_{C}(c)=\sum \varphi_{C \otimes C}\left(c_{(1)} \otimes c_{(2)}\right)=\sum \varphi_{C \otimes C} \circ l_{c_{(1)}}\left(c_{(2)}\right) \\
& =\sum\left(l_{c_{(1)}} \underset{C}{\square} \prod_{D} M\right) \circ \varphi_{C}\left(c_{(2)}\right)=\sum c_{(1)} \otimes \varphi_{C}\left(c_{(2)}\right) \text {. }
\end{aligned}
$$

Hence $\sigma_{E}=\varphi_{C}$ is a $C$-bicomodule map. A similar method can be used to show that $\tau_{E}$ is an $E$-bicomodule map. By the properties of the unit and counit of adjunction, the composition

$$
C \underset{C}{\square} N \stackrel{\varphi_{C} \underset{C}{\square} N}{\longrightarrow} C \underset{C}{\square} N \underset{E}{\square} M \underset{C}{\square} N \stackrel{v_{C \square} N}{\longrightarrow} C \underset{C}{\square} N
$$

yields the identity. Since $v$ is a natural transformation, the commutative diagrams induced by the morphisms $\rho^{N}, l_{n}: E \rightarrow N \otimes E, x \mapsto n \otimes x$, and ${ }^{N} \rho$, give the following equalities

$$
\begin{gathered}
v_{N \otimes E} \circ\left(\rho^{N} \underset{E}{\square} M \underset{c}{\square} N\right)=\rho^{N} \circ v_{N} \\
v_{N \otimes E} \circ\left(l_{n} \underset{E}{\square} M \square_{C} N\right)=l_{n} \circ v_{E}
\end{gathered}
$$


respectively. Hence, for all $n \otimes m \otimes n^{\prime} \in N \square_{E} M \square_{C} N$ (summation suppressed for simplicity),

$$
\begin{aligned}
\rho^{N} \circ v_{N}\left(n \otimes m \otimes n^{\prime}\right) & =\sum v_{N \otimes E} \circ\left(n_{[0]} \otimes n_{[1]} \otimes m \otimes n^{\prime}\right) \\
& =\sum v_{N \otimes E} \circ\left(l_{n_{[0]}}\left(n_{[1]}\right) \otimes m \otimes n^{\prime}\right) \\
& =\sum l_{n_{[0]}} \circ v_{E}\left(n_{[1]} \otimes m \otimes n^{\prime}\right)=\sum n_{[0]} \otimes v_{E}\left(n_{[1]} \otimes m \otimes n^{\prime}\right)
\end{aligned}
$$

where the first equality is from (3.4) and last by (3.5). And so applying $N \otimes \varepsilon_{E}$ to both sides and using the canonical identification $N \square E \simeq N$, we obtain $v_{N}=N \otimes \widehat{\tau}_{E}$, where $\widehat{\tau}_{E}:=$ $\varepsilon_{E} \circ \tau_{E}$. Since $\sigma_{E}=\varphi_{C}$, the first of relations (3.2) follows by the fact that the composition (3.3) is the identity. The other condition in (3.2) is proven in a similar way.

With this knowledge we can now state a proof for Theorem 3.2.6.

Proof. (of Theorem 3.2.6). By Proposition 1.3.14 there exists a unique coalgebra map $\pi: E \rightarrow D$ such that ${ }^{M} \rho=(\pi \otimes M) \circ \varphi_{M}$. Combining this with the matrix ring context $\left(C, E,{ }^{C} N^{E},{ }^{E} M^{C}, \sigma_{E}, \tau_{E}\right)$ constructed in Lemma 3.2.7 it is possible to define the required matrix ring context by

$$
\sigma: C \stackrel{\sigma_{E}}{\longrightarrow} N \underset{E}{\square} M \rightarrow N \underset{D}{\square} M, \quad \tau: M \square_{C} N \stackrel{\tau_{E}}{\longrightarrow} E \stackrel{\pi}{\rightarrow} D .
$$

It is immediate that these are bimodule maps and that they satisfy equation (3.2) is easily verified, for example

$$
\begin{aligned}
(N \otimes \widehat{\tau}) \circ\left(\sigma \square \square_{C} N\right) \circ{ }^{N} \rho & =\left(N \otimes\left(\varepsilon_{D} \circ \pi \circ \tau_{E}\right)\right) \circ\left(\sigma_{E} \otimes N\right) \circ{ }^{N} \rho \\
& =\left(N \otimes \widehat{\tau}_{E}\right) \circ\left(\sigma_{E} \otimes N\right) \circ{ }^{N} \rho=N .
\end{aligned}
$$

Where the second equality follows since $\pi$ is counital and the final equality since

$$
\left(C, E,{ }^{C} N^{E},{ }^{E} M^{C}, \sigma_{E}, \tau_{E}\right)
$$

is a matrix ring context.

It will also be useful to observe the following.

Proposition 3.2.8. Suppose that a right $C$-comodule $M$ is a quasi-finite injector, define $N:=h_{C}(M, C), E:=h_{C}(M, M)$ and let $\left(C, E,{ }^{C} N^{E},{ }^{E} M^{C}, \sigma_{E}, \tau_{E}\right)$ be the corresponding matrix ring context, as constructed in Proposition 3.2.7. Then by Proposition 1.3.18 there is an isomorphism $E \simeq M \square N$. Under this identification the induced coalgebra structure on $M \square_{C} N$ can be written as

$$
\Delta_{M \square_{C} N}=M \square_{C} \sigma_{E} \square_{C} N, \quad \varepsilon_{M \square_{C} N}=\widehat{\tau}_{E} .
$$

Proof. By the definition of the coendomorphism coalgebra the coproduct for $M \square N$ should be the unique map satisfying the condition

$$
\left(\Delta_{M} \underset{C}{\square_{N}} \otimes M\right) \circ \varphi_{M}=\left(M \square_{C} N \otimes \varphi_{M}\right) \circ \varphi_{M}
$$


Recall from the proof of Proposition 3.2.4

$$
\varphi_{M}:=(M \underset{C}{\square} \sigma) \circ \rho^{M}
$$

so we can verify

$$
\begin{aligned}
\left(\left((M \otimes \sigma \otimes N) \circ\left(\rho^{M} \otimes N\right)\right) \otimes M\right) \circ \varphi_{M} & =\left(\left((M \otimes \sigma) \circ \rho^{M}\right) \otimes N \otimes M\right) \circ(M \otimes \sigma) \circ \rho^{M} \\
& =(M \otimes \sigma \otimes \sigma) \circ\left(\rho^{M} \otimes C\right) \circ \rho^{M} \\
& =\left(M \otimes\left((\sigma \otimes \sigma) \circ \Delta_{C}\right)\right) \circ \rho^{M} \\
& =\left(M \otimes\left((N \otimes M \otimes \sigma) \circ\left(N \otimes \rho^{M}\right) \circ \sigma\right)\right) \circ \rho^{M} \\
& =\left(M \otimes N \otimes \varphi_{M}\right) \circ \varphi_{M} .
\end{aligned}
$$

Where the fourth equality follows since $\sigma$ is a $C$-bicolinear map. Hence the coproduct has the stated form. It is possible to check the counit in a similar way. $\square$

Remark 3.2.9. The notion of a matrix ring context has a very natural interpretation in the language of bicategories. Consider the bicategory of bicomodules where 0 -cells are coalgebras, 1-cells are bicomodules and 2-cells are bicomodule maps. Define the composite, $g \circ f$, of two 1-cells $f: X \rightarrow Y$ and $g: Y \rightarrow Z$ to be $f \square_{Y} g: X \rightarrow Z$. Then there are obvious associativity and unit isomorphisms. When the isomorphisms implicitly used in Definition 3.2.1, such as $\left(N \square_{D} M\right) \square_{C} N \cong N \square_{D}\left(M \square_{C} N\right)$, are fully described it becomes apparent that in this language $(C, D, g: C \rightarrow D, f: D \rightarrow C, \sigma, \tau)$ is a matrix ring context if and only if the 2-cells $\sigma: 1_{C} \Rightarrow f \circ g$ and $\tau: g \circ f \Rightarrow 1_{D}$ form an adjoint pair in the bicategory.

\subsubsection{Infinite (firm) matrix contexts.}

In the paper [27] the notion of a comatrix coring was generalized to allow for firm rings. In this section we follow their approach in the dual setting. This will lead to a more general theory in which we are not forced to consider comodules which are quasi-finite injectors.

Firstly recall a non-counital coalgebra $\mathrm{D}$ is said to be a firm coalgebra provided that the coproduct $\Delta_{D}: D \rightarrow D \square_{D} D$ is an isomorphism. Denoting the inverse of $\Delta_{D}$ by $\nabla_{D}: D \square_{C} D \rightarrow$ $D$ we are able to define firm comodules for a firm coalgebra as follows. A right (resp. left) non-counital $D$-comodule $M$ is firm provided $\rho^{M}: M \rightarrow M \square_{D} D$ (resp. ${ }^{M} \rho: M \rightarrow D \square_{D} M$ ) is an isomorphism of comodules. In calculations we shall denote the inverse of the coaction by $\nabla_{M}$ (resp. ${ }_{M} \nabla$ ). It will be useful to note that if $X$ is a firm right $D$-comodule and $Y$ a firm left $D$ comodule then

$$
\left(\nabla_{X} \square_{D} Y\right)=\left(X \square_{C} \nabla\right)
$$

To see this apply $\rho^{X} \square_{D} Y=X \square_{D}^{Y} \rho$ to both sides of the equation.

Definition 3.2.10. An infinite matrix ring context, $\left(C, D,{ }^{C} N^{D},{ }^{D} M^{C}, \sigma, \tau\right)$, consists of a counital coalgebra $C$, firm coalgebra $D$, a $(C, D)$-bicomodule $N$, a $(D, C)$-bicomodule $M$, both counital as $C$-comodules and firm as $D$-comodules, and a pair of bicomodule maps

$$
\sigma: C \rightarrow N \square_{D} M, \quad \tau: M \square_{C} N \rightarrow D
$$

such that the diagrams in Definition 3.2.1 commute. 
In the case of an infinite matrix ring context we do not assume the existence of a counit for $D$, therefore we are unable to form a reduced counit as for matrix ring contexts. However assuming $N$ is firm right $D$-comodule we are able to replace instances of $N \otimes \varepsilon_{D}$ with $\nabla_{N}$. A similar substitution can be made for left $D$-comodules. Therefore in terms of $\nabla_{N}$ and ${ }_{M} \nabla$, the commutative diagrams in Definition 3.2.1 read

$$
\nabla_{N} \circ(N \square \underset{D}{\square} \tau) \circ(\sigma \underset{C}{\square} N) \circ{ }^{N} \rho=N, \quad{ }_{M} \nabla \circ(\tau \underset{D}{\square} M) \circ(M \underset{C}{\square} \sigma) \circ \rho^{M}=M .
$$

Likewise we are able to adapt the proof of Proposition 3.2.4 to associate an adjoint pair of functors to an infinite matrix ring context.

Proposition 3.2.11. Given an infinite matrix ring context $\left(C, D,{ }^{C} N^{D},{ }^{D} M^{C}, \sigma, \tau\right)$, denote by $\mathbf{M}^{D}$ the category of firm right $D$-comodules. Then the functor $F=-\square_{C} N: \mathbf{M}^{C} \rightarrow \mathbf{M}^{D}$ is the left adjoint of $G=-\square_{D} M: \mathbf{M}^{D} \rightarrow \mathbf{M}^{C}$.

Proof. Define natural transformations

$$
\begin{gathered}
\varphi: \mathbf{M}^{C} \rightarrow G F, \quad \varphi_{X}:=(X \underset{C}{\square} \sigma) \circ \rho^{X}, \\
v: F G \rightarrow \mathbf{M}^{D}, \quad v_{Y}:=\nabla_{Y} \circ\left(Y \square_{D} \tau\right)
\end{gathered}
$$

Then check these that these morphisms are the unit and counit, respectively, of the adjunction. Firstly take any right $C$-comodule $\mathrm{X}$ and compute

$$
\begin{aligned}
v_{F(X)} \circ F\left(\varphi_{X}\right) & =\nabla_{X} \square_{C} \circ\left(X \square_{C} N \square_{D} \tau\right) \circ\left(\left((X \underset{C}{\square} \sigma) \circ \rho^{X}\right) \square_{C} N\right) \\
& =\nabla_{X} \square_{C} \circ\left(X \underset{C}{\square} N \square_{D} \tau\right) \circ\left(X \square_{C} \sigma \square_{C}^{N} \rho\right) \\
& =\left(X \underset{C}{\square} \nabla_{N}\right) \circ(X \underset{C}{\square} N \underset{D}{\square} \tau) \circ\left(X \underset{C}{\square} \sigma \square_{C} N\right) \circ\left(X \square_{C}^{N} \rho\right) \\
& =X \square_{C} N=F(X) .
\end{aligned}
$$

Where the second equality follows the definition of the cotensor product and the third since it is clear that $\left(X \square \nabla_{N}\right)$ is the inverse of $\rho^{X{ }_{C} N}=X \square \rho_{C} \rho^{N}$. The fourth equality the follows from the equations (3.8). On the other hand, for all firm right $D$-comodules $\mathrm{Y}$,

$$
\begin{aligned}
G\left(v_{Y}\right) \circ \varphi_{G(Y)} & =\left(\left(\nabla_{Y} \circ\left(Y \square_{D} \tau\right)\right) \underset{D}{\square} M\right) \circ\left(Y \underset{D}{\square} M \square_{C} \sigma\right) \circ \rho^{Y \square_{D} M} \\
& =\left(Y \underset{D}{\square}\left(M \nabla \circ\left(\tau \square_{D} M\right)\right)\right) \circ(Y \underset{D}{\square} M \underset{C}{\square} \sigma) \circ\left(Y \square_{D} \rho^{M}\right) \\
& =Y \square_{D} M=G(Y) .
\end{aligned}
$$

Where the second equality follows by the relation (3.7) and the third by the second of the equations (3.8).

Note that since we do not know that $\nabla_{N}: N \square_{D} D \rightarrow N$ extends naturally to a function on $N \otimes D$, we do not know that this adjoint pair of functors extends to functors between $\mathbf{M}^{C}$ and Vect $_{k}$. Therefore $\mathrm{M}$ is no longer forced to be a quasi-finite injector as a right $C$-comodule. However we are still able to associate a firm coalgebra and a $C$-ring to an infinite matrix ring context. 
Proposition 3.2.12. Let $\left(C, D,{ }^{C} N^{D},{ }^{D} M^{C}, \sigma, \tau\right)$ be an infinite matrix ring context.

(1) $\mathscr{A}:=N \underset{D}{\square} M$ is a $C$-ring with the product and unit

$$
\mu_{\mathscr{A}}=\left(\nabla_{N} \square \square_{D} M\right) \circ\left(N \underset{D}{\square} \tau \square_{D} M\right)=\left(N \square_{D} \nabla\right) \circ\left(N \underset{D}{\square} \tau \square_{D} M\right), \quad \eta_{\mathscr{A}}=\sigma .
$$

(2) $E:=M \underset{C}{\square} N$ is a firm coalgebra with the coproduct

$$
\Delta_{E}=\left(M \underset{c}{\square} \sigma \square_{C} N\right) \circ\left(\rho^{M} \square_{C} N\right)=\left(M \underset{C}{\square} \sigma \square_{C} N\right) \circ\left(M \square_{C}^{N} \rho\right) .
$$

Proof. (1) Since $\nabla_{N}$ is the inverse of a $(C, D)$-bicomodule map, it must be a $(C, D)$ bicomodule map. Hence $\mu_{\mathscr{A}}$ is a composition of $C$-bicomodule maps and therefore a $C$ bicomodule map. That the two forms for the product are equivalent follows from equation (3.7). For the associativity of $\mu_{\mathscr{A}}$ first compute

$$
\begin{aligned}
& \left.\mu_{\mathscr{A}} \circ\left(\mu_{\mathscr{A}} \square_{C} \mathscr{A}\right)=\left(N \underset{D}{\square_{M}} \nabla\right) \circ(N \underset{D}{\square} \tau \underset{D}{\square} M) \circ\left(\left(\nabla_{N} \square_{D} M\right) \circ\left(N \underset{D}{\square} \tau \square_{D} M\right)\right) \square_{C} N \square_{D} M\right) \\
& =\left(\nabla_{N} \square_{D} M\right) \circ\left(N \underset{D}{\square} D \square_{D} \tau \square_{D} M\right) \circ\left(N \underset{D}{\square} \tau \square_{D} M \square_{C} N \square_{D} M\right) \\
& =\left(\nabla_{N} \square_{D} M \nabla\right) \circ\left(N \underset{D}{\square} \tau \underset{D}{\square} \tau \square_{D} M\right) \text {. }
\end{aligned}
$$

On the other hand

$$
\begin{aligned}
& \mu_{\mathscr{A}} \circ\left(\mathscr{A} \square_{C} \mu_{\mathscr{A}}\right)=\left(\nabla_{N} \square_{D} M\right) \circ\left(N \underset{D}{\square} \tau \square_{D} M\right) \circ\left(N \square_{D} M \square_{C}\left(\left(N \square_{D} \nabla\right) \circ\left(N \underset{D}{\square} \tau \square_{D} M\right)\right)\right) \\
& =\left(\nabla_{N} \square_{D} M\right) \circ\left(N \square_{D} \tau \square_{D} D \square_{D} M\right) \circ\left(N \underset{D}{\square} M \square_{C} N \square_{D} \tau \square_{D} M\right) \\
& =\left(\nabla_{N} \square_{D} M\right) \circ\left(N \square_{D} \tau \square_{D} \tau \square_{D} M\right) \text {. }
\end{aligned}
$$

That $\eta_{\mathscr{A}}$ is a $C$-bicolinear is clear. Finally that $\mu_{\mathscr{A}}$ is unital with respect to $\eta_{\mathscr{A}}$ follows from the equations (3.8).

(2) The map $\Delta_{E}$ is coassociative since

$$
\begin{aligned}
& \left(\Delta_{E} \otimes E\right) \circ \Delta_{E}=\left(\left(\left(M \square_{c} \sigma \square_{c} N\right) \circ\left(\rho^{M} \square_{c} N\right)\right) \otimes M \square_{c} N\right) \circ\left(M \square_{c} \sigma \square_{c} N\right) \circ\left(\rho^{M} \square_{c} N\right) \\
& =\left(M \underset{c}{\square} \sigma \square_{C} N \otimes M \square_{c} N\right) \circ\left(M \square_{c} C \square_{c} \sigma \square_{c} N\right) \circ\left(\left(\left(\rho^{M} \square_{c} C\right) \circ \rho^{M}\right) \underset{c}{\square_{C}} N\right) \\
& =\left(M \square_{C} N \otimes M \square_{C} \sigma \square_{C} N\right) \circ\left(M \square_{C} \sigma \square_{C} C \square_{C} N\right) \circ\left(\left(\left(M \square_{C} \Delta_{C}\right) \circ \rho^{M}\right) \square_{C} N\right) \\
& =\left(M \square_{C} N \otimes M \square_{C} \sigma \square_{C} N\right) \circ\left(M \square_{C}\left(\left(N \otimes \rho^{M}\right) \circ \sigma\right) \square_{C} N\right) \circ\left(\rho^{M} \square_{C} N\right) \\
& =\left(E \otimes \Delta_{E}\right) \circ \Delta_{E} \text {. }
\end{aligned}
$$

Where the third equality follows by the coassociativity of $\rho^{M}$ and the fourth by the colinearity of $\sigma$. To see that $\Delta_{E}: E \rightarrow E \square_{E} E$ is an isomorphism define

$$
\nabla_{E}: E \underset{E}{\square} E \rightarrow E, \quad \nabla_{E}=\left({ }_{M} \nabla \underset{C}{\square} N\right) \circ\left(\tau \underset{D}{\square} M \square_{C} N\right)
$$

Now note that the canonical $D$-bicomodule structure on $E$ is induced from the coproduct $\Delta_{E}$ via the comultiplicative map $\tau: E \rightarrow D$, therefore $E \underset{E}{\square} E \subseteq E \underset{D}{\square} E$. After making this observation we can compute that

$$
\nabla_{E} \circ \Delta_{E}=\left({ }_{M} \nabla \underset{C}{\square} N\right) \circ(\tau \underset{D}{\square} M \underset{C}{\square} N) \circ\left(M \underset{C}{\square} \sigma \square_{C} N\right) \circ\left(\rho^{M} \underset{C}{\square} N\right)=M \underset{C}{\square} N=E,
$$


by the second of the equations (3.8). On the other hand

$$
\begin{aligned}
\Delta_{E} \circ \nabla_{E} & =\left(M \square_{C} \sigma \square_{C} N\right) \circ\left(M \square_{C}^{N} \rho\right) \circ\left(M \nabla \square_{C} N\right) \circ\left(\tau \square_{E} M \square_{C} N\right) \\
& =\left({ }_{M} \nabla \square_{C} N \square_{D} M \square_{C} N\right) \circ\left(D \square_{D} M \square_{C} \sigma \square_{C} N\right) \circ\left(D \square_{D} M \square_{C}^{N} \rho\right) \circ\left(\tau \square_{E} M \square_{C} N\right) \\
& =\left({ }_{M} \nabla \square_{C} N \square_{D} M \square_{C} N\right) \circ\left(\tau \square_{D} M \square_{C} N \square_{D} M \square_{C} N\right) \circ\left(M \square_{C} N \square_{E} \Delta_{E}\right) \\
& =\left({ }_{M} \nabla \square_{C} N \square_{D} M \square_{C} N\right) \circ\left(\tau \square_{D} M \square_{C} N \square_{D} M \square_{C} N\right) \circ\left(\Delta_{E} \square_{E} M \square_{C} N\right) \\
& =\left(\nabla_{E} \square_{E} M \square_{C} N\right) \circ\left(\Delta_{E} \square_{E} M \square_{C} N\right)=E \square_{E} E .
\end{aligned}
$$

Where the fourth equality follows by the definition of $-\square_{E}-$ and the final equality by the previous calculation. Hence $\nabla_{E}$ is the inverse of $\Delta_{E}$ and $E$ is a firm coalgebra.

Remark 3.2.13. In the case of a quasi-finite injective comodule $M^{C}$, Proposition 3.2.8 implies the coalgebra $E$ defined above coincides with the coendomorphism coalgebra.

\section{3 $\mathscr{A}$-coendomorphism coalgebra and Galois modules}

In this section we develop the notion of Galois modules for a $C$-ring. We then give a structure theorem for these modules and also construct an associated Galois connection.

\subsubsection{The $\mathscr{A}$-coendomorphism coalgebra and $C$-ring}

In order to develop this theory we must first associate to a module of a $C$-ring a coalgebra. Such a coalgebra should be thought of as a dual object to an endomorphism ring. To achieve this we restrict ourselves to modules which are quasi-finite injectors as $C$ comodules and adapt the associated coendomorphism coalgebra to take into account the action of the $C$-ring.

Lemma 3.3.1. Let $\left(C, D,{ }^{C} N^{D},{ }^{D} M^{C}, \sigma, \tau\right)$ be a matrix ring context and let $\mathscr{A}$ be a $C$-ring. If $M$ is a right $\mathscr{A}$-module, via the map $\overline{\rho_{M}}: M \square_{C} \mathscr{A} \rightarrow M$, then $N$ is a left $\mathscr{A}$-module via the map

$$
\bar{N} \rho: \mathscr{A} \square_{C} N \rightarrow N, \quad \bar{N} \rho:=(N \otimes \widehat{\tau}) \circ\left(N \square_{D} \overline{\rho_{M}} \square_{C} N\right) \circ\left(\sigma \square_{C} \mathscr{A} \square_{C} N\right) \circ\left({ }^{\mathscr{A}} \rho \square_{C} N\right)
$$

Proof. It is clear that since $\bar{N} \rho$ is a composition of left $C$-colinear maps, $\bar{N} \bar{\rho}$ is left $C$-colinear. In order to check that $\bar{N} \rho$ is associative and unital, we adopt the following conventions. Firstly we write $\sigma(c)=\sum c^{[1]} \otimes c^{[2]} \in N \square_{D} M$. Secondly we denote the right action $\overline{\rho_{M}}(m \otimes a)$ as $m \triangleleft a$, and the left action $\overline{N \rho}(a \otimes m)=a \triangleright m$. In this notation

$$
\sum_{i} a^{i} \triangleright n^{i}=\sum_{i} a_{[-1]}^{i]} \widehat{\tau}\left(a_{[-1]}^{i}{ }_{[2]}^{[2]} a_{[0]}^{i} \otimes n^{i}\right), \quad \text { for all } \sum_{i} a^{i} \otimes n^{i} \in \mathscr{A} \square \square_{C} N
$$


Now we are able to verify that $\bar{N} \rho$ satisfies the required associativity condition by taking any $a \otimes a^{\prime} \otimes n \in \mathscr{A} \square_{C} \mathscr{A} \square_{C} N$ (summation suppressed) and computing

$$
\begin{aligned}
& \left(a \triangleright\left(a^{\prime} \triangleright n\right)\right)=\sum a_{[-1]}^{[1]} \widehat{\tau}\left(a_{[-1]}^{[2]} \triangleleft a_{[0]} \otimes a_{[-1]}^{\prime}{ }^{[1]}\right) \widehat{\tau}\left(a_{[-1]}^{\prime[2]} \triangleleft a_{[0]}^{\prime} \otimes n\right) \\
& =\sum a_{[-1]}{ }^{[1]} \widehat{\tau}\left(a_{[-1]}{ }^{[2]} \triangleleft a_{[0]} \otimes a_{[1]}{ }^{[1]}\right) \widehat{\tau}\left(a_{[1]}^{[2]} \triangleleft a^{\prime} \otimes n\right) \\
& =\sum a_{[-1]}^{[1]} \widehat{\tau}\left(\left(a_{[-1]}^{[2]} \triangleleft a_{[0]}\right)_{[0]} \otimes\left(a_{[-1]}{ }^{[2]} \triangleleft a_{[0]}\right)_{[1]}^{[1]}\right) \\
& \times \widehat{\tau}\left(\left(a_{[-1]^{[2]}} \triangleleft a_{[0]}\right)_{[1]}^{[2]} \triangleleft a^{\prime} \otimes n\right) \\
& =\sum a_{[-1]}^{[1]} \widehat{\tau}\left(\left(a_{[-1]}{ }^{[2]} \triangleleft a_{[0]}\right) \triangleleft a^{\prime} \otimes n\right) \\
& =\sum a_{[-1]}^{[1]} \widehat{\tau}\left(\left(a_{[-1]}{ }^{[2]} \triangleleft\left(a_{[0]} a^{\prime}\right)\right) \otimes n\right) \\
& =\sum\left(a a^{\prime}\right)_{[-1]}^{[1]} \widehat{\tau}\left(\left(\left(a a^{\prime}\right)_{[-1]}^{[2]} \triangleleft\left(a a^{\prime}\right)_{[0]}\right) \otimes n\right)=\left(\left(a a^{\prime}\right) \triangleright n\right) \text {. }
\end{aligned}
$$

Where the second equality follows since $a \otimes a^{\prime} \in \mathscr{A} \square{ }_{c} \mathscr{A}$ and the third by the right $C$ colinearity of the right $\mathscr{A}$-action. Then the fourth equality follows from the second of the equations (3.2). Finally the penultimate equality holds because of the colinearity of the product $\mu_{\mathscr{A}}$. This proves the associativity. As for the unitality, take $n \in N$ and compute

$$
\begin{aligned}
\sum \eta_{\mathscr{A}}\left(n_{[-1]}\right) \triangleright n_{[0]} & =\sum\left(\eta_{\mathscr{A}}\left(n_{[-1]}\right)\right)_{[-1]}{ }^{[1]} \widehat{\tau}\left(\left(\eta_{\mathscr{A}}\left(n_{[-1]}\right)\right)_{[-1]}{ }^{[2]} \triangleleft\left(\eta_{\mathscr{A}}\left(n_{[-1]}\right)\right)_{[0]} \otimes n_{[0]}\right) \\
& =\sum n_{[-2]}^{[1]} \widehat{\tau}\left(n_{[-2]}{ }^{[2]} \triangleleft \eta_{\mathscr{A}}\left(n_{[-1]}\right) \otimes n_{[0]}\right) \\
& =\sum n_{[-1]}^{[1]} \widehat{\tau}\left(n_{[-1]}{ }^{[2]}{ }_{[0]} \triangleleft \eta_{\mathscr{A}}\left(n_{[-1]}{ }_{[1]}^{[2]}\right) \otimes n_{[0]}\right) \\
& =\sum n_{[-1]}^{[1]} \widehat{\tau}\left(n_{[-1]}{ }^{[2]} \otimes n_{[0]}\right)=n .
\end{aligned}
$$

Where the second and third equalities follow by the left $C$-colinearity of $\eta_{\mathscr{A}}$ and $\sigma$ respectively. Then the fourth equality holds since $\overline{\rho_{M}}$ is unital and finally the last equality follows by the first of the equations (3.2).

Before defining what an $\mathscr{A}$-coendomorphism coalgebra is, we first need to observe the following.

Lemma 3.3.2. Let $\left(C, D,{ }^{C} N^{D},{ }^{D} M^{C}, \sigma, \tau\right)$ be a matrix ring context and let $\mathscr{A}$ be a $C$-ring. Suppose that $M$ is a right $\mathscr{A}$-module, via the map $\overline{\rho_{M}}: M \square_{C} \mathscr{A} \rightarrow M$ (denoted by $\triangleleft$ between elements) and let $\overline{N \rho}$ be the left $\mathscr{A}$-action on $N$ constructed in Lemma 3.3 .1 (denoted by $\triangleright$ between elements).

(1) For all $m \otimes a \otimes n \in M \square_{C} \mathscr{A} \square_{C} N$ (summation suppressed for clarity),

$$
\widehat{\tau}(m \triangleleft a \otimes n)=\widehat{\tau}(m \otimes a \triangleright n) .
$$

(2) The following diagram

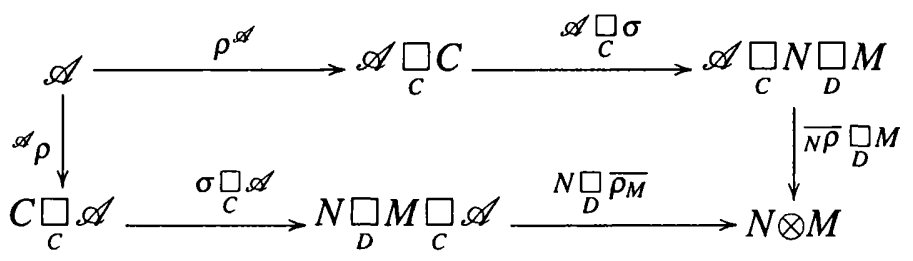

is commutative. 
Proof.

(1) Take any $m \otimes a \otimes n \in M \square_{C} \mathscr{A} \square_{C} N$ (summation suppressed) and compute

$$
\begin{aligned}
\widehat{\tau}(m \otimes a \triangleright n) & =\sum \widehat{\tau}\left(m \otimes a_{[-1]}{ }^{[1]} \widehat{\tau}\left(a_{[-1]}^{[2]} \triangleleft a_{[0]} \otimes n\right)\right) \\
& =\sum \widehat{\tau}\left(m_{[0]} \otimes m_{[1]}^{[1]} \widehat{\tau}\left(m_{[1]}{ }^{[2]} \triangleleft a \otimes n\right)\right) \\
& =\sum \widehat{\tau}\left(\widehat{\tau}\left(m_{[0]} \otimes m_{[1]}^{[1]}\right) m_{[1]}^{[2]} \triangleleft a \otimes n\right) \\
& =\widehat{\tau}(m \triangleleft a \otimes n),
\end{aligned}
$$

where the second equality is satisfied because $m \otimes a \in M \square \mathscr{c}$, the third by the $k$-linearity of $\widehat{\tau}$ and finally the last equality follows from the second of the equations (3.2).

(2) Take any $a \in \mathscr{A}$ and compute

$$
\begin{aligned}
& \sum a_{[0]} \triangleright a_{[1]}^{[1]} \otimes a_{[1]}^{[2]} \\
& =\sum a_{[-1]}{ }^{[1]} \widehat{\tau}\left(a_{[-1]}{ }^{[2]} \triangleleft a_{[0]} \otimes a_{[1]}^{[1]}\right) \otimes a_{[1]}^{[2]} \\
& =\sum a_{[-1]}^{[1]} \widehat{\tau}\left(\left(a_{[-1]}{ }^{[2]} \triangleleft a_{[0]}\right)_{[0]} \otimes\left(a_{[-1]}^{[2]} \triangleleft a_{[0]}\right)_{[1]}^{[1]}\right) \otimes\left(a_{[-1]}^{[2]} \triangleleft a_{[0]}\right)_{[1]}^{[2]} \\
& =\sum a_{[-1]}^{[1]} \otimes a_{[-1]}^{[2]} \triangleleft a_{[0]} \text {, }
\end{aligned}
$$

where the second equality follows by the $C$-colinearity of the right $\mathscr{A}$-action and the third from the second of the equations (3.2).

Theorem 3.3.3. Let $\mathscr{A}$ be $a C$-ring and $M$ be a right $\mathscr{A}$-module which is a quasi-finite injector as a right $C$-comodule. Define $N:=h_{C}(M, C), E:=h_{C}(M, M)$ and $E_{\mathscr{A}}(M)$ to be the vector space given by the coequalizer

$$
M \square_{C} \mathscr{A} \square_{C} N \underset{\frac{\overline{\rho_{M}} \square_{C} N}{M \square_{C} \overline{N \rho}}}{\longrightarrow} M \square_{C} N \longrightarrow \stackrel{\pi_{\mathscr{A}}}{\longrightarrow} E_{\mathscr{A}}(M)
$$

in which $\overline{\rho_{M}}$ is the right $\mathscr{A}$-action on $M$ and $\bar{N} \rho$ the corresponding left $\mathscr{A}$-action on $N$ induced from $\left(C, E,{ }^{C} N^{E},{ }^{E} M^{C}, \sigma_{E}, \tau_{E}\right)$ (as constructed in Lemma 3.2.7) in the way described in Lemma 3.3.1. After making the identification of coalgebras $E \simeq M \square N$ as described Proposition 3.2.8, $E_{\mathscr{A}}(M)$ can be given a coalgebra structure such that

$$
M \underset{C}{\square} N \stackrel{\pi_{\mathscr{A}}}{\longrightarrow} E_{\mathscr{A}}(M)
$$

is a coalgebra map. The coalgebra $E_{\mathscr{A}}(M)$ is called an $\mathscr{A}$-coendomorphism coalgebra of $M$.

Proof. Since $M^{C}$ is assumed to be quasi-finite injective, by Proposition 3.2 .8 the identification $E \simeq M \square_{C} N$ induces a coalgebra structure on $M \square_{C} N$ with coproduct and counit which can be written as

$$
\Delta_{M \square_{C} N}=M \underset{C}{\square} \sigma_{E} \square_{C} N, \quad \varepsilon_{M} \square_{C} N=\widehat{\tau}_{E}
$$


Now by the previous lemma it is clear that the counit factors through $\pi_{\mathscr{A}}$. For the comultiplication, writing $\pi_{\mathscr{A}}(m \otimes n)=[m \otimes n]$ for all $m \otimes n \in M \square_{C} N$ and $\sigma_{E}(c)=\sum c^{[1]} \otimes c^{[2]}$ for all $c \in C$, take any $m \otimes a \otimes n \in M \square_{C} \mathscr{A} \square_{C} N$ (summation suppressed) and compute

$$
\begin{aligned}
\Delta_{E_{\mathscr{A}}(M)}([m \otimes a \triangleright n]) & =\left(\pi_{\mathscr{A}} \otimes \pi_{\mathscr{A}}\right) \circ \Delta_{M \square_{C} N}(m \otimes a \triangleright n) \\
& =\sum\left[m_{[0]} \otimes m_{[1]}[1]\right] \otimes\left[m_{[1]}{ }^{[2]} \otimes a \triangleright n\right] \\
& =\sum\left[m \otimes a_{[-1]}[1]\right] \otimes\left[a_{[-1]}{ }^{[2]} \triangleleft a_{[0]} \otimes n\right] \\
& =\sum\left[m \otimes a_{[0]} \triangleright a_{[1]}^{[1]}\right] \otimes\left[a_{[1]}^{[2]} \otimes n\right] \\
& =\sum\left[m \triangleleft a_{[0]} \otimes a_{[1]}^{[1]}\right] \otimes\left[a_{[1]}^{[2]} \otimes n\right] \\
& =\sum\left[(m \triangleleft a)_{[0]} \otimes(m \triangleleft a)_{[1]}^{[1]}\right] \otimes\left[(m \triangleleft a)_{[1]}^{[2]} \otimes n\right] \\
& =\Delta_{E_{\mathscr{A}}(M)}([m \triangleleft a \otimes n]) .
\end{aligned}
$$

Where the third equality uses the assumption $m \otimes a \otimes n \in M \square_{C} \mathscr{A} \square_{C} N$ and the defining property of $\pi_{\mathscr{A}}$. The fourth equality follows by part (2) of Lemma 3.3.2. Then the fifth equality again uses the defining property of $\pi_{\mathscr{A}}$. Finally the sixth equality follows by the right $C$-colinearity of $\overline{\rho_{M}}$. This demonstrates that the coalgebra structure on $M \square N$ descends onto $E_{\mathscr{A}}(M)$ and the associated projection $\pi_{\mathscr{A}}$ is a coalgebra map.

Corollary 3.3.4. Let $\mathscr{A}$ be $a C$-ring and $M$ a right $\mathscr{A}$-module which is a quasi-finite injector as a $C$-comodule, and let $N:=h_{C}(M, C)$. Denote by ${ }^{N} \rho$ the induced left $C$-coaction on $N$, as described in Proposition 1.3.7. Then

(1) $M$ is an $\left(E_{\mathscr{A}}(M), C\right)$ - bicomodule, with left coaction $\left(\pi_{\mathscr{A}} \otimes M\right) \circ\left(M \square_{C} \sigma_{E}\right) \circ \rho^{M}$.

Furthermore this left coaction is right $\mathscr{A}$-linear.

(2) $N$ is a $\left(C, E_{\mathscr{A}}(M)\right)$ - bicomodule, with right coaction $\left(N \otimes \pi_{\mathscr{A}}\right) \circ\left(\sigma_{E} \square_{C} N\right) \circ{ }^{N} \rho$. Furthermore this right coaction is left $\mathscr{A}$-linear.

Proof. By Proposition 1.3.14, $M$ is an $(E, C)$ bicomodule with the left coaction given by the unit of adjunction for the adjoint pair $\left(h_{C}(M,-),-\otimes M\right), \varphi_{M}: M \rightarrow h_{C}(M, M) \otimes M$. Now by Lemma 3.2 .7 we can construct a matrix ring context $\left(C, E,{ }^{C} N^{E},{ }^{E} M^{C}, \sigma_{E}, \tau_{E}\right)$, and in terms of these maps $\varphi_{M}=\left(M \square{ }_{C} \sigma_{E}\right) \circ \rho^{M}$ (see proof of Proposition 3.2.4). Then since $\pi_{\mathscr{A}}$ is a coalgebra map, it induces a left $E_{\mathscr{A}}$-coaction on $M$ which is right $C$-colinear. Therefore $M$ is an $\left(E_{\mathscr{A}}(M), C\right)$-bicomodule with the prescribed left coaction. To see that the left coaction is right $\mathscr{A}$-linear take $m \otimes a \in M \square_{C} \mathscr{A}$, and writing $\pi_{\mathscr{A}}(m \otimes n)=[m \otimes n]$, compute

$$
\begin{aligned}
\left(\pi_{\mathscr{A}} \otimes M\right) \circ\left(M \square{ }_{C} \sigma_{E}\right) \circ \rho^{M}(m \triangleleft a) & =\sum\left[(m \triangleleft a)_{[0]} \otimes(m \triangleleft a)_{[1]}{ }^{[1]}\right] \otimes(m \triangleleft a)_{[1]}^{[2]} \\
& =\sum\left[m \triangleleft a_{[0]} \otimes a_{[1]}^{[1]}\right] \otimes a_{[1]}[2] \\
& =\sum\left[m \otimes a_{[0]} \triangleright a_{[1]}^{[1]}\right] \otimes a_{[1]}[2] \\
& =\sum\left[m \otimes a_{[-1]}^{[1]}\right] \otimes a_{[-1]}^{[2]} \triangleleft a_{[0]} \\
& =\sum\left[m_{[0]} \otimes m_{[1]}^{[1]}\right] \otimes m_{[1]}^{[2]} \triangleleft a \\
& =\left(\left(\pi_{\mathscr{A}} \otimes M\right) \circ\left(M \square_{C} \sigma_{E}\right) \circ \rho^{M}(m)\right) \triangleleft a .
\end{aligned}
$$


Where the second equality follows from the right $C$-colinearity of the right $\mathscr{A}$-action, the third by the the defining property of $\pi_{\mathscr{A}}$ and the forth by part (2) of Lemma 3.3.2. Finally the fifth equality holds since $m \otimes a \in M \square_{C} \mathscr{A}$. For the the second part of the corollary observe that since we can construct a matrix ring context $\left(C, E,{ }^{C} N^{E},{ }^{E} M^{C}, \sigma_{E}, \tau_{E}\right), N$ must be a quasi-finite injector as a left $C$-module, by a left handed version of Proposition 3.2.4. We can then complete the proof in a way similar to the first part.

Therefore to any right $\mathscr{A}$-module $M$ which is a quasi-finite injector as a right $C$ comodule we are able to associate a matrix ring context

$$
\left(C, E_{\mathscr{A}}(M),{ }^{C} N^{E_{\mathscr{A}}(M)}, E_{\mathscr{A}}(M) M^{C}, \sigma, \tau\right)
$$

which is induced from $\left(C, E,{ }^{C} N^{E},{ }^{E} M^{C}, \sigma_{E}, \tau_{E}\right)$ by the map $\pi_{\mathscr{A}}$.

Definition 3.3.5. The matrix ring context $\left(C, E_{\mathscr{A}}(M),{ }^{C} N^{E_{\mathscr{A}}(M)}, E_{\mathscr{A}}(M) M^{C}, \sigma, \tau\right)$ described above is called the $\mathscr{A}$-coendomorphism coalgebra context of $M$ and the associated matrix $C$-ring the $\mathscr{A}$-coendomorphism ring of $M$.

\subsubsection{Galois and principal modules}

Using the constructions of the previous subsection we now describe a special class of modules for a $C$-ring and as the main result of this subsection give a structure theorem for such modules.

Proposition 3.3.6. Let $\mathscr{A}$ be $a C$-ring and $M$ a right $\mathscr{A}$-module which is a quasi-finite injector as a $C$-comodule. Set $N:=h_{C}(M, C)$ and define

$$
\beta: \mathscr{A} \rightarrow N \otimes M, \quad \beta:=\left(N \otimes \overline{\rho_{M}}\right) \circ\left(\sigma \square \mathscr{A}_{C}\right) \circ^{\mathscr{A}} \rho
$$

where $\sigma$ is the unit of the corresponding $\mathscr{A}$-coendomorphism context associated to $M$ and $\overline{\rho_{M}}$ signifies the right $\mathscr{A}$-action on $M$. Then, writing $S$ for the coalgebra $E_{\mathscr{A}}(M)$,

(1) $\beta(\mathscr{A}) \subset N \square M$.

(2) The map $\beta$ is a morphism of $C$-rings.

Proof. Using the conventions of writing

(a) $\sigma(c)=\sum c^{[1]} \otimes c^{[2]}$, for all $c \in C$,

(b) $\pi_{\mathscr{A}}(m \otimes n)=[m \otimes n]$, for all $m \otimes n \in M \square_{C} N$ (summation suppressed),

(c) $\overline{\rho_{M}}(m \otimes a)=m \triangleleft a$, for all $m \otimes a \in M \square \underset{C}{\square} \mathscr{A}$ (summation suppressed),

(d) $\bar{N} \rho(a \otimes m)=a \triangleright m$, for all $a \otimes m \in \mathscr{A} \square_{C} M$ (summation suppressed),

(e) ${ }^{M} \rho$ and $\rho^{N}$ for the $E_{\mathscr{A}}(M)$ coactions described in Corollary 3.3.4,

we are able to write $\beta(a)=\sum a_{[-1]}^{[1]} \otimes a_{[-1]^{[2]}} \triangleleft a_{[0]}$ for all elements $a \in \mathscr{A}$. Using this notation we can then proceed as follows. 
(1) To show that $\beta(\mathscr{A}) \subset N \underset{S}{\square} M$ we compute

$$
\begin{aligned}
\left(N \otimes{ }^{M} \rho\right)(\beta(a)) & =\sum a_{[-1]^{[1]}}{ }^{[1]}\left[a_{[-1]^{[2]}} \triangleleft a_{[0]} \otimes a_{[1]}^{[1]}\right] \otimes a_{[1]}^{[2]} \\
& =\sum a_{[-1]^{[1]}}\left[_{[-1]}^{[2]} \otimes a_{[0]} \triangleright a_{[1]}^{[1]}\right] \otimes a_{[1]}^{[2]} \\
& =\sum a_{[-2]^{[1]}}\left[_{[-2]^{[2]}} \otimes a_{[-1]^{[1]}}\right] \otimes a_{[-1]}^{[2]} \triangleleft a_{[0]}=\left(\rho^{N} \otimes M\right)(\beta(a)),
\end{aligned}
$$

where the first equality follows from the right $C$ colinearity of the right $\mathscr{A}$-action, the second by the defining property of $\pi_{\mathscr{A}}$ and the third by part (2) of Lemma 3.3.2. Then the last equality follows by the left $C$-colinearity of $\sigma$.

(2) Firstly it is clear that $\beta$ is $C$-bimodule map since it is easily seen to be a composition of $C$-bimodule maps. To see that the map $\beta$ respects the unit, take any $c \in C$ and compute

$$
\begin{aligned}
\beta \circ \eta_{\mathscr{A}}(c) & =\left(N \square_{D} \overline{\rho_{M}}\right) \circ\left(\sigma \square_{c} \mathscr{A}\right) \circ{ }^{\mathscr{A}} \rho \circ \eta(c) \\
& =\sum\left(N \square_{D} \overline{\rho_{M}}\right) \circ\left(\sigma \square \square_{C} \mathscr{A}\right)\left(c_{(1)} \otimes \eta\left(c_{(2)}\right)\right) \\
& =\sum\left(N \square_{D} \overline{\rho_{M}}\right)\left(c_{(1)}{ }^{[1]} \otimes c_{(1)}{ }^{[2]} \otimes \eta\left(c_{(2)}\right)\right) \\
& =\sum\left(N \square_{D} \overline{\rho_{M}}\right)\left(c^{[1]} \otimes c_{[0]}^{[2]} \otimes \eta\left(c^{[2]}{ }_{[1]}\right)\right)=\sigma(c) .
\end{aligned}
$$

Where the second equality is by the left $C$-colinearity of $\eta$, the fourth equality is by the left $C$ colinearity of $\sigma$ and the final equality follows since $\overline{\rho_{M}}$ is unital. This shows that $\beta$ is unital since $\sigma$ is the unit of the $\mathscr{A}$-coendomorphism $C$-ring $N \square_{S} M$. To see that $\beta$ is multiplicative take any $a \otimes a^{\prime} \in \mathscr{A} \square_{C} \mathscr{A}$ (summation suppressed) and denoting the product in $\mathscr{A}$ by juxtaposition, compute

$$
\begin{aligned}
& \mu_{N \underset{S}{ } M}\left(\beta(a) \underset{C}{\square} \beta\left(a^{\prime}\right)\right)=\sum a_{[-1]}^{[1]} \widehat{\tau}\left(a_{[-1]}^{[2]} \triangleleft a_{[0]} \otimes a_{[-1]}^{\prime[1]}\right) \otimes a_{[-1]}^{\prime}{ }^{[2]} \triangleleft a_{[0]}^{\prime} \\
& =\sum a_{[-1]}{ }^{[1]} \widehat{\tau}\left(a_{[-1]}^{[2]} \triangleleft a_{[0]} \otimes a_{[1]}^{[1]}\right) \otimes a_{[1]}^{[2]} \triangleleft a^{\prime} \\
& =\sum a_{[-1]}^{[1]} \widehat{\tau}\left(\left(a_{[-1]}^{[2]} \triangleleft a_{[0]}\right)_{[0]} \otimes\left(a_{[-1]}^{[2]} \triangleleft a_{[0]}\right)_{[1]}^{[1]}\right) \\
& \otimes\left(a_{[-1]}^{[2]} \triangleleft a_{[0]}\right)_{[1]}^{[2]} \triangleleft a^{\prime} \\
& =\sum a_{[-1]}^{[1]} \otimes\left(a_{[-1]}^{[2]} \triangleleft a_{[0]}\right) \triangleleft a^{\prime} \\
& =\sum a_{[-1]^{[1]}} \otimes a_{[-1]}^{[2]} \triangleleft a_{[0]} a^{\prime} \\
& =\sum\left(a a^{\prime}\right)_{[-1]}^{[1]} \otimes\left(a a^{\prime}\right)_{[-1]}^{[2]} \triangleleft\left(a a^{\prime}\right)_{[0]} \\
& =\beta\left(a a^{\prime}\right) \text {. }
\end{aligned}
$$

Where the second equality is because $a \otimes a^{\prime} \in \mathscr{A} \square \square_{C} \mathscr{A}$, the third is by the right $C$-colinearity of the action of $\mathscr{A}$ and follows from the equations (3.2). Finally the penultimate equality is from the left $C$-colinearity of the product in $\mathscr{A}$.

Definition 3.3.7. Let $\mathscr{A}$ be a $C$-ring and $M$ a right $\mathscr{A}$-module which is a quasi-finite injector as a $C$-comodule. Set $N:=h_{C}(M, C)$ and $S:=E_{\mathscr{A}}(M)$ to be the $\mathscr{A}$-coendomorphism coalgebra of $M$. Then $M$ is called a Galois $\mathscr{A}$-module if and only if the map $\beta: \mathscr{A} \rightarrow$ $N \square_{S} M$, as defined in Proposition 3.3.6 is bijective. If in addition $M$ is injective as a left $S$-module then $M$ is said to be principal. 
In fact just as Galois comodules generalize Galois corings, we now show that the Galois modules we have just defined generalize Galois $C$-rings, as defined in [4, section 6]. Firstly recall the notion dual to a grouplike element

Definition 3.3.8. Let $\mathscr{A}$ be a $C$-ring then $\kappa: \mathscr{A} \rightarrow k$ is said to be a non-trivial character provided it is multiplicative and satisfies $\kappa \circ \eta_{\mathscr{A}}=\varepsilon_{C}$.

Lemma 3.3.9. For any $C$-ring $\mathscr{A}$ there is a one to one correspondence between right $\mathscr{A}$ actions on $C$ and non-trivial characters $\kappa: \mathscr{A} \rightarrow k$.

Proof. Firstly given a right $\mathscr{A}$-action $\overline{\rho_{C}}: C \square \mathscr{A}_{C} \rightarrow C$ define a non-trivial character

$$
\kappa\left[\overline{\rho_{C}}\right]: \mathscr{A} \rightarrow k, \quad \kappa\left[\overline{\rho_{C}}\right]:=\varepsilon_{C} \circ \overline{\rho_{C}} \circ^{\mathscr{A}} \rho .
$$

To see that $\kappa\left[\overline{\rho_{C}}\right]$ is multiplicative take any $a \otimes a^{\prime} \in \mathscr{A} \square{ }_{C} \mathscr{A}$ (summation suppressed) and compute, writing $\overline{\rho_{C}}(m \otimes a)=m \triangleleft a$ for all $m \otimes a \in M \square_{C}^{C} \mathscr{A}$ (summation suppressed) and denoting the product in $\mathscr{A}$ by juxtaposition,

$$
\begin{aligned}
\kappa\left[\overline{\rho_{C}}\right]\left(a a^{\prime}\right) & =\sum \varepsilon_{C}\left(\left(a a^{\prime}\right)_{[-1]} \triangleleft\left(a a^{\prime}\right)_{[0]}\right) \\
& =\sum \varepsilon_{C}\left(a_{[-1]} \triangleleft a_{[0]} a^{\prime}\right) \\
& =\sum \varepsilon_{C}\left(\left(a_{[-1]} \triangleleft a_{[0]}\right) \triangleleft a^{\prime}\right),
\end{aligned}
$$

by using the left $C$-colinearity of $\mu_{\mathscr{A}}$ and then the associativity of $\overline{\rho_{C}}$. On the other hand we can also compute that

$$
\begin{aligned}
\kappa\left[\overline{\rho_{C}}\right](a) \kappa\left[\overline{\rho_{C}}\right]\left(a^{\prime}\right) & =\sum \varepsilon_{C}\left(a_{[-1]} \triangleleft a_{[0]}\right) \varepsilon_{C}\left(a_{[-1]}^{\prime} \triangleleft a_{[0]}^{\prime}\right) \\
& =\sum \varepsilon_{C}\left(a_{[-1]} \triangleleft a_{[0]}\right) \varepsilon_{C}\left(a_{[1]} \triangleleft a^{\prime}\right) \\
& =\sum \varepsilon_{C}\left(\left(a_{[-1]} \triangleleft a_{[0]}\right)_{(1)}\right) \varepsilon_{C}\left(\left(a_{[-1]} \triangleleft a_{[0]}\right)_{(2)} \triangleleft a^{\prime}\right) \\
& =\sum \varepsilon_{C}\left(\left(a_{[-1]} \triangleleft a_{[0]}\right) \triangleleft a^{\prime}\right),
\end{aligned}
$$

where the second equality follows because $a \otimes a^{\prime} \in \mathscr{A} \square \square_{C}$, the third by the right $C$ colinearity of $\overline{\rho_{C}}$ and the final equality since $\varepsilon_{C}$ is the counit for $C$. Therefore $\kappa\left[\overline{\rho_{C}}\right]$ is multiplicative. Similarly a straight forward calculation, using the $C$-colinearity of $\eta_{\mathscr{A}}$ and the unitality of $\overline{\rho_{C}}$, shows that $\kappa \circ \eta_{\mathscr{A}}=\varepsilon_{C}$ for all $c \in C$. Therefore for any right $\mathscr{A}$-action $\overline{\rho_{C}}: C \square, \mathscr{A} \rightarrow C$ the map $\kappa\left[\overline{\rho_{C}}\right]: \mathscr{A} \rightarrow k$ is a non-trivial character.

On the other hand given a non-trivial character $\kappa: \mathscr{A} \rightarrow k$ we can define a right $\mathscr{A}$ coaction on $C$

$$
\overline{\rho_{C}}[\kappa]: C \square_{C} \mathscr{A} \rightarrow C, \quad \overline{\rho_{C}}[\kappa]:=\left(\varepsilon_{C} \square_{C} \kappa \square_{C} C\right) \circ\left(C \square_{C} \rho^{\mathscr{A}}\right) .
$$

It is a routine calculation, which we omit for brevity, to show that this is indeed a right $\mathscr{A}$ action on $C$. That the correspondence given in (3.10) and (3.9) is one-to-one can also easily be checked, for example

$$
\kappa\left[\overline{\rho_{M}}[\kappa]\right]=\varepsilon_{C} \circ\left(\varepsilon_{C} \square_{C} \kappa \square_{C} C\right) \circ\left(C \square_{C} \rho^{\mathscr{A}}\right) \circ \mathscr{A}^{\mathscr{A}} \rho=\left(\varepsilon_{C} \square_{C} \kappa \square_{C} \varepsilon_{C}\right) \circ\left(C \square_{C} \rho^{\mathscr{A}}\right) \circ \mathscr{A}^{\mathscr{A}} \rho=\kappa .
$$

Where the second equality follows by the $k$-linearity of $\varepsilon_{C}$ and the final equality by the counitality of the maps ${ }^{\mathscr{A}} \rho$ and $\rho^{\mathscr{A}}$. 
Lemma 3.3.10. For any $C$-ring $\mathscr{A}$ with a nontrivial character $\kappa: \mathscr{A} \rightarrow k$ the set

$$
I_{\kappa}:=\left\{\sum \kappa\left(a_{[0]}\right) a_{[1]}-\sum a_{[-1]} \kappa\left(a_{[0]}\right) \mid a \in \mathscr{A}\right\} \subseteq C,
$$

is a coideal of $C$.

Proof. Observe that for any $a \in \mathscr{A}$ and $\beta_{1}, \beta_{2} \in\left\{\beta \in \operatorname{Hom}_{k}(C, k) \mid \beta\left(I_{\kappa}\right)=0\right\}$, writing * for the convolution product in $\operatorname{Hom}_{k}(C, k)$,

$$
\begin{aligned}
\sum \kappa\left(a_{[0]}\right)\left(\beta_{1} * \beta_{2}\right)\left(a_{[1]}\right) & =\sum \kappa\left(a_{[0]}\right) \beta_{1}\left(a_{[1]}\right) \beta_{2}\left(a_{[2]}\right) \\
& =\sum \beta_{1}\left(a_{[-1]}\right) \kappa\left(a_{[0]}\right) \beta_{2}\left(a_{[1]}\right) \\
& =\sum \beta_{1}\left(a_{[-2]}\right) \beta_{2}\left(a_{[-1]}\right) \kappa\left(a_{[0]}\right) \\
& =\sum\left(\beta_{1} * \beta_{2}\right)\left(a_{[-1]}\right) \kappa\left(a_{[0]}\right) .
\end{aligned}
$$

Where the second equality follows since $\beta_{1}\left(I_{K}\right)=0$ and the third since $\beta_{2}\left(I_{\kappa}\right)=0$. Therefore $\left\{\beta \in \operatorname{Hom}_{k}(C, k) \mid \beta\left(I_{K}\right)=0\right\}$ is a subalgebra of the convolution algebra $\operatorname{Hom}_{k}(C, k)$ and so, by [36, Proposition 1.4.6], $I_{\kappa}$ must be a coideal of $C$.

Definition 3.3.11. Let $\mathscr{A}$ be a $C$-ring with nontrivial character $\kappa$. Then we call $B_{\kappa}:=C / I_{\kappa}$ the coalgebra of coinvariants.

Proposition 3.3.12. Let $\mathscr{A}$ be a $C$-ring, then every non-trivial character $\kappa: \mathscr{A} \rightarrow k$ induces a map of $C$-rings

$$
\Gamma_{\kappa}: \mathscr{A} \rightarrow C \underset{B_{K}}{\square} C, \quad \Gamma_{\kappa}:=\left(C \underset{C}{\square} \kappa \square_{C} C\right) \circ\left(C \underset{C}{\square} \rho^{\mathscr{A}}\right) \circ^{\mathscr{A}} \rho
$$

where $C \underset{B_{K}}{\square} C$ is the Sweedler type $C$-ring arising from the natural coextension $C \rightarrow B_{\kappa}$.

Proof. First recall that in the Sweedler type $C$-ring arising from the natural coextension $C \rightarrow B_{\kappa}$, the left and right $B_{\kappa}$ coactions on $C$ are those induced by the canonical projection $\pi: C \rightarrow C / I_{\kappa}$. That the map $\Gamma_{\kappa}$ is well defined, i.e. $\operatorname{Im} \Gamma_{\kappa} \subseteq C \square_{B_{K}} C$, then follows since, for all $a \in \mathscr{A}$,

$$
\sum a_{[-2]} \otimes \pi\left(a_{[-1]} \kappa\left(a_{[0]}\right)\right) \otimes a_{[1]}=\sum a_{[-1]} \otimes \pi\left(\kappa\left(a_{[0]}\right) a_{[1]}\right) \otimes a_{[2]},
$$

by the linearity of $\pi$ and the definition of the coideal $I_{\kappa}$. Since $\Gamma_{\kappa}$ is a composition of $C$-bicolinear maps, it must be $C$-bicolinear. To see that $\Gamma_{\kappa}$ is unital take any $c \in C$ and compute

$$
\begin{aligned}
\Gamma_{\kappa} \circ \eta_{\mathscr{A}}(c) & =\sum\left(\eta_{\mathscr{A}}(c)\right)_{[-1]}\left(\kappa \circ\left(\eta_{\mathscr{A}}(c)_{[0]}\right)\right) \otimes\left(\eta_{\mathscr{A}}(c)\right)_{[1]} \\
& =\sum c_{(1)}\left(\kappa \circ\left(\eta_{\mathscr{A}}\left(c_{(2)}\right)\right)\right) \otimes c_{(3)} \\
& =\sum c_{(1)} \otimes c_{(2)}=\eta_{C_{B_{K}} C}(c)
\end{aligned}
$$

where the second equality follows since $\eta_{\mathscr{A}}$ is bicolinear and the third since $\kappa \circ \eta_{\mathscr{A}}=\varepsilon_{C}$. That $\Gamma_{K}$ is a multiplicative map is easily confirmed by a straight forward calculation using the fact that the product in $\mathscr{A}$ is $C$-bicolinear and the multiplicativity of $\kappa$. 
Definition 3.3.13. Let $\mathscr{A}$ be a $C$-ring with a nontrivial character $\kappa$. Then we say that $\mathscr{A}$ is a Galois $C$-ring (with respect to $\kappa$ ) if and only if the map $\Gamma_{\kappa}: \mathscr{A} \rightarrow C \square_{B_{K}} C$, as defined in (3.12), is an isomorphism.

Proposition 3.3.14. Let $\mathscr{A}$ be a $C$-ring with a nontrivial character $\kappa: \mathscr{A} \rightarrow k$. Then $\mathscr{A}$ is a Galois $C$-ring (with respect to $\kappa$ ) if and only if $C$ is a Galois right $\mathscr{A}$-module (with the right $\mathscr{A}$-action induced by $\kappa)$.

Proof. First observe that for every coalgebra $C$ it is possible to associate a matrix ring context $\left(C, C,{ }^{C} C^{C},{ }^{C} C^{C}, \sigma, \tau\right)$, where $\tau: C \square_{C} C \rightarrow C$ and $\sigma: C \rightarrow C \square_{C} C$ are the obvious isomorphisms. Therefore from Proposition 3.2.4 it is clear that $C$ is quasi-finite as a right $C$ comodule and $h_{C}(C, C)=C$. By Proposition 3.3.9, $C$ is a right $\mathscr{A}$-module with right action $\overline{\rho_{C}}(c \otimes a)=\sum \varepsilon_{C}(c) \kappa\left(a_{[0]}\right) a_{[1]}$ and the corresponding left $\mathscr{A}$-action, given by Lemma 3.3.2, is $\bar{c} \rho(a \otimes c)=\sum a_{[-1]} \kappa\left(a_{[0]}\right) \varepsilon_{C}(c)$. In view of this

$$
S=C \square_{C} C / \operatorname{Im}\left(\overline{\rho_{C}} \square_{C} C-C \square_{C} \overline{C \rho}\right) \simeq C /\left\{\sum \kappa\left(a_{(0)}\right) a_{(1)}-\sum a_{(-1)} \kappa\left(a_{(0)}\right) \mid a \in \mathscr{A}\right\}=B_{\kappa},
$$

where the middle isomorphism is induced by the natural isomorphism $C \square C \simeq C$. It then follows that $C \square_{B_{K}} C=C \square_{S} C$, but also observe that for all $a \in \mathscr{A}$,

$$
\begin{aligned}
\beta(a) & =\left(N \underset{D}{\square_{C}} \overline{\rho_{C}}\right) \circ\left(\sigma \square_{C} \mathscr{A}\right) \circ^{\mathscr{A}} \rho \\
& =\sum a_{[-2]} \otimes \overline{\rho_{C}}\left(a_{[-1]} \otimes a_{[0]}\right) \\
& =\sum a_{[-2]} \otimes \varepsilon_{C}\left(a_{[-1]}\right) \kappa\left(a_{[0]}\right) a_{[1]} \\
& =\sum a_{[-1]} \otimes \kappa\left(a_{[0]}\right) a_{[1]}=\Gamma_{\kappa}(a) .
\end{aligned}
$$

Therefore in this situation $\Gamma_{\kappa}: \mathscr{A} \rightarrow C \square C$ and $\beta: \mathscr{A} \rightarrow C \square C$ are precisely the same maps. Therefore if one is an isomorphism of $C$-rings so is the other and the result follows.

Proposition 3.3.15. If $M$ is a right principal Galois module for a $C$-ring $\mathscr{A}$ then $\mathscr{A}$ is injective as a left $C$-comodule.

Proof. Suppose $M$ is a principal $\mathscr{A}$-module. Define $N:=h_{C}(M, C)$ and $S:=E_{\mathscr{A}}$, and let $E=M \square \subset$ denote the coendomorphism coalgebra of $M$. Since $M$ is Galois there is an isomorphism $\mathscr{A} \simeq N \square_{S} M$ and furthermore a chain of isomorphisms

$$
N \square_{E} M \square_{C} \mathscr{A} \simeq N \square_{E} M \square_{C} N \square_{S} M \simeq N \square_{E} E \square_{S} M \simeq N \square \square_{S} M \simeq \mathscr{A} .
$$

Explicitly, following this chain from right to left the isomorphism $\mathscr{A} \rightarrow N \square_{E} M \square_{C} \mathscr{A}$ is given by the composition

$$
\begin{aligned}
\left.\left(N \underset{E}{\square} M \underset{C}{\square} \beta^{-1}\right) \circ\left(\left(\left(\sigma_{E} \square_{C} N\right) \circ{ }^{N} \rho\right)\right)\right) & \square_{S} M \circ \beta \\
= & \left(N \square_{E} M \square_{C} \beta^{-1}\right) \circ\left(\sigma_{E} \square_{C} N \square_{S} M\right) \circ\left(C \square_{C} \beta\right) \circ \mathscr{A}^{\mathscr{A}} \rho \\
= & \left(\sigma_{E} \square_{C} \mathscr{A}\right) \circ \mathscr{A} \rho,
\end{aligned}
$$


where $\sigma_{E}$ is the unit of the matrix ring context described in Lemma 3.2.7 and the first equality holds since $\beta$ is left $C$-colinear. Next observe that since $M$ is assumed to be injective as a left $S$-comodule $E \square_{S} M$ is injective as a left $E$-comodule (see [14, Subsection 11.11]). Therefore in view of the existence of the chain of isomorphism

$$
M \square_{C} \mathscr{A} \simeq M \square_{C} N \square_{S} M \simeq E \square_{S} M
$$

$M \square_{C} \mathscr{A}$ is injective as a left $E$-comodule. Thus there exists a map $p$ which is a retraction for the obvious inclusion $i: M \square_{C} \mathscr{A} \rightarrow M \otimes A$. Since it is then clear that that $N \square_{E} p$ is a left $C$-colinear retraction of $N \square_{E} i$ there is a commutative diagram with (split) exact rows

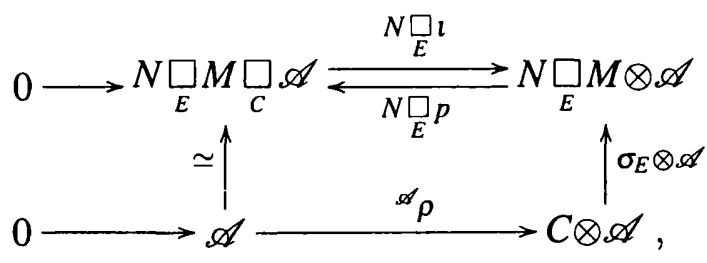

from which it is apparent that the map ${ }^{\mathscr{A}} \rho$ has a left $C$-colinear retraction. Therefore $\mathscr{A}$ is injective as a left $C$-comodule.

We now give the main result of this section

Theorem 3.3.16. Let $\mathscr{A}$ be $a C$-ring and $M$ a right $\mathscr{A}$-module which is a quasi-finite injector as a right $C$-comodule. Set $N=h_{C}(M, C)$ and $S=E_{\mathscr{A}}(M)$. View $N \otimes M$ and $N \square_{S} M$ as left $\mathscr{A}$-modules with the left action as in Lemma 3.3.1. Let $\beta$ be as in Proposition 3.3.6. Then

1. The following statements are equivalent

(a) there exists a left $\mathscr{A}$-module map $\chi: N \otimes M \rightarrow \mathscr{A}$ such that $\chi \circ \beta=\mathscr{A}$ (i.e. $\beta: \mathscr{A} \rightarrow N \otimes M$ is a split monomorphism of left $\mathscr{A}$-modules);

(b) $M$ is a principal Galois $\mathscr{A}$-module.

2. The following statements are equivalent

(a) there exists a left $\mathscr{A}$-module map $\hat{\chi}: N \square{ }_{s} M \rightarrow \mathscr{A}$ such that $\hat{\chi} \circ \beta=\mathscr{A}$ (i.e. $\beta: \mathscr{A} \rightarrow N \square M$ is a split monomorphism of left $\mathscr{A}$-modules);

(b) $M$ is a Galois $\mathscr{A}$-module.

Proof. (1) (a) $\Rightarrow$ (b) Assume that $\chi$ is a left $\mathscr{A}$-linear retraction of $\beta$. Then for every $a \in \mathscr{A}, \sum \chi\left(\sigma\left(a_{[-1]}\right) \triangleleft a_{[0]}\right)=a$, where $\sigma$ is the unit of the $\mathscr{A}$-coendomorphism ring context of $M$. In particular for every $c \in C$, writing $\sigma(c)=\sum c^{[1]} \otimes c^{[2]}$,

$$
\eta_{\mathscr{A}}(c)=\sum \chi\left(\sigma\left(c_{(1)}\right) \triangleleft \eta_{\mathscr{A}}\left(c_{(2)}\right)\right)=\sum \chi\left(c^{[1]} \otimes c^{[2]}{ }_{[0]} \triangleleft \eta_{\mathscr{A}}\left(c^{[2]}{ }_{[1]}\right)\right)=\chi \circ \sigma(c),
$$


where the first equality follows by the left $C$-colinearity of $\eta_{\mathscr{A}}$, the second by the right $C$-colinearity of $\sigma$ and the third equality since the right $\mathscr{A}$ action on $M$ is unital. Therefore

$$
\chi \circ \sigma=\eta_{\mathscr{A}}
$$

After making this observation we now proceed to demonstrate that $M$ is injective as a left $S$-module, by constructing a left $S$-comodule retraction of the left $S$-coaction on M. Define $\delta: S \otimes M \rightarrow M$ to be the map the satisfying the commutative diagram

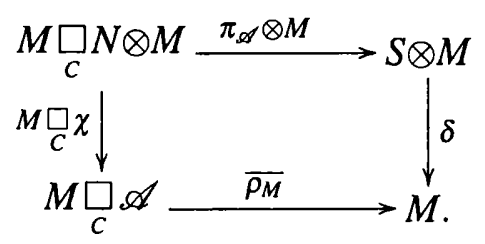

This map is well defined since $\chi$ is assumed to be left $\mathscr{A}$-linear and therefore the definition of $\delta$ is independent of the choice of pre-image under the map $\pi_{\mathscr{A}}$. Using equation (3.13) and the unitality of the right $\mathscr{A}$-action we can compute that, for all $m \in M$,

$$
\delta \circ{ }^{M} \rho(m)=\sum m_{[0]} \triangleleft \chi\left(\sigma\left(m_{[1]}\right)\right)=\sum m_{[0]} \triangleleft \eta_{\mathscr{A}}\left(m_{[1]}\right)=m .
$$

Therefore $\delta$ is a retraction of the left $S$-coaction on M. Furthermore since ${ }^{M} \rho$ is right $\mathscr{A}$ linear (see Proposition 3.3.4), for all $m \otimes n \in M \square_{C} N$ and $m^{\prime} \in M$, writing $\pi_{\mathscr{A}}(m \otimes n)=$ $[m \otimes n]$, we can compute

$$
\begin{aligned}
(S \otimes \delta) \circ\left(\Delta_{S} \otimes M\right)\left([m \otimes n] \otimes m^{\prime}\right) & =\sum\left[m_{[0]} \otimes m_{[1]}{ }^{[1]}\right] \otimes \delta\left(\left[m_{[1]}[2] \otimes n\right] \otimes m^{\prime}\right) \\
& =\sum\left[m_{[0]} \otimes m_{[1]}{ }^{[1]}\right] \otimes m_{[1]}[2] \triangleleft \chi\left(n \otimes m^{\prime}\right) \\
& ={ }^{M} \rho(m) \triangleleft \chi\left(n \otimes m^{\prime}\right)={ }^{M} \rho\left(m \triangleleft \chi\left(n \otimes m^{\prime}\right)\right) \\
& ={ }^{M} \rho \circ \delta\left([m \otimes n] \otimes m^{\prime}\right) .
\end{aligned}
$$

To see that the first equality holds recall that $\pi_{\mathscr{A}}$ is a coalgebra map and the coalgebra structure of $E \simeq M \square_{C} N$ given in Proposition 3.2.8. This shows that $\delta$ is left $S$-colinear and therefore completes the proof that $M$ is an injective left $S$-comodule.

Next we show that $\beta$ is an isomorphism. Define $\hat{\beta}=\left.\chi\right|_{N \square}$, since $\operatorname{Im} \beta \subseteq M \square N$ and $\chi$ is a retraction of $\beta$, it is clear that $\hat{\beta} \circ \beta=\mathscr{A}$. Moreover $\hat{\beta}$ is right inverse of $\beta$, to see this take any $n \otimes m \in N \square_{S} M$ and compute

$$
\begin{aligned}
\beta \circ \hat{\beta}(n \otimes m) & =\sum \sigma\left(\hat{\beta}(n \otimes m)_{[-1]}\right) \triangleleft \hat{\beta}(n \otimes m)_{[0]}=\sum \sigma\left(n_{[-1]}\right) \triangleleft \hat{\beta}\left(n_{[0]} \otimes m\right) \\
& =\sum n \otimes m_{[0]} \triangleleft \hat{\beta}\left(\sigma\left(m_{[1]}\right)\right)=\sum n \otimes m_{[0]} \triangleleft \eta_{\mathscr{A}}\left(m_{[1]}\right)=n \otimes m .
\end{aligned}
$$

Where the second equality follows since $\chi$ is left $\mathscr{A}$-linear, which requires that $\chi$ is a left $C$-colinear map. To see that the third equality let $\lambda: S \square \square_{S} M \rightarrow M$ be the unique map satisfying

$$
\lambda \circ\left(\pi_{\mathscr{A}} \underset{S}{\square} M\right)=\overline{\rho_{M}} \circ(M \underset{C}{\square} \hat{\beta}) .
$$


Such a map can defined since $\pi_{\mathscr{A}}$ is surjective and $\hat{\beta}$ is left $\mathscr{A}$-linear. Now recall that $n \otimes m \in N \square M$ and therefore $\sum\left(N \otimes \pi_{\mathscr{A}} \otimes M\right)\left(\sigma\left(n_{[-1]}\right) \otimes n_{[0]} \otimes m-n \otimes m_{[0]} \otimes \sigma\left(m_{[1]}\right)\right)=0$, applying $N \otimes \lambda$ to this equality gives the third equality. Then the fourth equality follows by equation (3.13) and the final final equality bye the unitality of $\overline{\rho_{M}}$. Hence $\hat{\beta}$ is an inverse of $\beta$ and so we are able to conclude that $M$ is a principal Galois $\mathscr{A}$-module.

(1) (b) $\Rightarrow$ (a) Suppose that $M$ is a principal Galois $\mathscr{A}$-module and let $\delta: S \otimes M \rightarrow M$ be a $S$-comodule retraction of ${ }^{M} \rho$. Then define $\chi: N \otimes M \rightarrow \mathscr{A}$ to be the composition of maps,

$$
N \otimes M \stackrel{\rho^{N} \otimes M}{\longrightarrow} N \square_{S} S \otimes M \stackrel{N \otimes \delta}{\longrightarrow} N \square_{S} M \stackrel{\beta^{-1}}{\longrightarrow} \mathscr{A}
$$

Since $\rho^{N}$ is left $\mathscr{A}$-linear (see Proposition 3.3.4) and $\beta^{-1}$ is assumed to be left $\mathscr{A}$-linear, $\chi$ is a composition of left $\mathscr{A}$-linear maps and therefore left $\mathscr{A}$-linear. Moreover we can compute,

$$
\chi \circ \beta=\beta^{-1} \circ(N \otimes \delta) \circ\left(\rho^{N} \otimes M\right) \circ \beta=\beta^{-1} \circ(N \otimes \delta) \circ\left(N \otimes{ }^{M} \rho\right) \circ \beta=\beta^{-1} \circ \beta=\mathscr{A},
$$

using the fact that $\operatorname{Im}(\beta) \in N \underset{s}{\square} M$ in the second equality. So we conclude $\chi$ is a left $\mathscr{A}$-linear retraction of $\beta$.

(2) that (b) $\Rightarrow$ (a) is trivial. To show (a) $\Rightarrow$ (b) the same method can be used as for $(1)(a) \Rightarrow(1)(b)$.

We shall later use this result to produce a generalization of Schneider's Theorem II.

\subsubsection{A Galois connection}

In [20] a way of associating a Galois connection to a comatrix coring was described. Using a similar method we now show how it is possible to associate a Galois connection to a matrix $C$-ring. In what follows we assume that $C$ is a coalgebra over a field $k$ and that $M \in \mathbf{M}^{C}$ is a quasi-finite injector. We then define $N:=h_{C}(M, C)$ and $E$ to be the coendomorphism coalgebra of $M$. Finally we let $\pi: E \rightarrow D$ be a coalgebra map, define $\mathscr{A}:=N \square \square$ to be the associated matrix $C$-ring and view $M$ as right $\mathscr{A}$-module by the action described in Proposition 3.2.2.

Firstly observe that for any subcoideal $X \subseteq \operatorname{ker} \pi, \pi$ factors through $E / X$ and therefore it is possible to define a matrix $C$-ring

$$
\mathscr{A}(X):=N \underset{E / X}{\square} M .
$$

Observe that for any subcoideal $Y \subseteq X$, the canonical coalgebra map $E / Y \rightarrow E / X$ induces an inclusion of $C$-rings $\mathscr{A}(Y) \subseteq \mathscr{A}(X)$. Since $\mathscr{A}(\operatorname{ker} \pi)=\mathscr{A}$, this implies that any subcoideal $Y \subseteq \operatorname{ker} \pi$ induces an inclusion of $C$-rings $\mathscr{A}(Y) \subseteq \mathscr{A}$.

Lemma 3.3.17. For any subcoideal $X \subseteq \operatorname{ker} \pi$,

$$
\operatorname{ker} \pi_{\mathscr{A}(X)} \subseteq X,
$$

where $\operatorname{ker} \pi_{\mathscr{A}(X)}$ is the epimorphism defining the $\mathscr{A}(X)$-coendomorphism coalgebra of $M$, as described in Theorem 3.3.3, and the right $\mathscr{A}(X)$-module structure on $M$ is as described in Proposition 3.2.2. 
Proof. First recall from Theorem 3.3.3 that $E_{\mathscr{A}(X)}(M)$ and $\pi_{\mathscr{A}(X)}$ are defined by the coequalizer

$$
M \square_{C} \mathscr{A}(X) \underset{C}{\square} N \underset{\overline{\rho_{M}} \square_{C} N}{\overline{M \square_{\bar{N}} \bar{\rho}}} M \square_{C} N \stackrel{\pi_{\mathscr{A}}}{\longrightarrow} E_{\mathscr{A}(X)}(M),
$$

where $\overline{\rho_{M}}$ and $\overline{N \rho}$ are as defined in Proposition 3.2.2. Also observe that $\bar{N} \rho$ coincides with the left $\mathscr{A}(X)$-action on $N$ obtained by feeding $\overline{\rho_{M}}$ into Lemma 3.3.1. Therefore $x \in \operatorname{ker} \pi_{\mathscr{A}(X)}$ if and only if there exists $m \otimes n \otimes m^{\prime} \otimes n^{\prime} \in M \square_{C} \mathscr{A}(X) \square_{C} N$ (summation suppressed) such that

$$
x=\widehat{\tau}_{E}(m \otimes n) m^{\prime} \otimes n^{\prime}-m \otimes n \widehat{\tau}_{E}\left(m^{\prime} \otimes n^{\prime}\right) .
$$

Writing $\pi_{X}: E \rightarrow E / X$ for the canonical coalgebra epimorphism, the $E / X$-coaction on $M$ and $N$ are

$$
{ }^{M} \rho(m)=\sum \pi_{X}\left(m_{[0]} \otimes m_{[1]}^{[1]}\right) \otimes m_{[1]}^{[2]}, \quad \rho^{N}(n)=\sum n_{[-1]}^{[1]} \otimes \pi_{X}\left(n_{[-1]}{ }^{[2]} \otimes n_{[0]}\right),
$$

where $\sigma_{E}(c)=\sum c^{[1]} \otimes c^{[2]}$, for all $c \in C$. Therefore if $m \otimes n \otimes m^{\prime} \otimes n^{\prime} \in M \underset{c}{\square} \mathscr{A}(X) \underset{c}{\square} N$, then

$$
\begin{aligned}
\widehat{\tau}_{E}(m \otimes n) \pi_{X}\left(m^{\prime} \otimes n^{\prime}\right) & =\sum \widehat{\tau}_{E}(m \otimes n) \pi_{X}\left(m^{\prime}{ }_{[0]} \otimes m^{\prime}{ }_{[1]}^{[1]}\right) \widehat{\tau}_{E}\left(m^{\prime}{ }_{[1]}^{[2]} \otimes n^{\prime}\right) \\
& =\sum \widehat{\tau}_{E}\left(m \otimes n_{[-1]}{ }^{[1]}\right) \pi_{X}\left(n_{[-1]}{ }^{[2]} \otimes n_{[0]}\right) \widehat{\tau}_{E}\left(m^{\prime} \otimes n^{\prime}\right) \\
& =\pi_{X}(m \otimes n) \widehat{\tau}_{E}\left(m^{\prime} \otimes n^{\prime}\right) .
\end{aligned}
$$

Where the first and third equalities follow since $\widehat{\tau}_{E}$ is the reduced counit of the coendomorphism context of $M$, and therefore satisfies the equations (3.2). The second equality holds since $m \otimes n \otimes m^{\prime} \otimes n^{\prime} \in M \square_{C} N \square_{E / X} M \square_{C} N$, in which the middle cotensor product is over $E / X$. Therefore for all $x \in \operatorname{ker} \pi_{\mathscr{A}(X)}, \pi_{X}(x)=0$ and so $\operatorname{ker} \pi_{\mathscr{A}(X)} \subseteq \operatorname{ker} \pi_{X}=X$.

Setting $X=\operatorname{ker} \pi_{X}$ in the previous lemma, we see that $\operatorname{ker} \pi_{\mathscr{A}} \subseteq \operatorname{ker}_{\pi}$, since $\mathscr{A}(\operatorname{ker} \pi)=$ $\mathscr{A}$. Now suppose $\mathscr{B}$ is a $C$-subring of $\mathscr{A}$ and $\pi_{\mathscr{B}}$ is the coalgebra map defining the $\mathscr{B}$ coendomorphism coalgebra of $M$. Then it is clear that $\operatorname{ker} \pi_{\mathscr{B}} \subset \operatorname{ker} \pi_{\mathscr{A}}$ and so we can define a corresponding subcoideal of $\operatorname{ker} \pi$,

$$
\mathscr{X}(\mathscr{B}):=\operatorname{ker} \pi_{\mathscr{B}}
$$

Where the right $\mathscr{B}$-action on $M$ is induced by the right $\mathscr{A}$-action via the inclusion. Note that if $\mathscr{B} \subseteq \mathscr{B}^{\prime}$ are $C$-subrings of $\mathscr{A}$, then $\mathscr{X}(\mathscr{B}) \subseteq \mathscr{X}\left(\mathscr{B}^{\prime}\right)$. Therefore we are able to give an order reversing correspondence between partially ordered sets

$$
\{C \text {-subrings of } \mathscr{A}\} \underset{\mathscr{A}(-)}{\stackrel{\mathscr{X}(-)}{\rightleftarrows}} \text { \{subcoideals of } \operatorname{ker} \pi\} \text {, }
$$

where the subcoideals are ordered by the relation $X^{\prime} \leq X$ if and only if $X \subseteq X^{\prime}$ and the $C$-subrings by inclusion. Moreover we shall now show that this correspondence is a Galois connection. 
Proposition 3.3.18. For all C-subrings $\mathscr{B} \subseteq \mathscr{A}$ and subcoideals $X \subseteq \operatorname{ker} \pi$,

(1) $\mathscr{B} \subseteq \mathscr{A}(\mathscr{X}(\mathscr{B}))$, and $\mathscr{B}=\mathscr{A}(\mathscr{X}(\mathscr{B}))$ if and only if $M$ is a Galois $\mathscr{B}$-module;

(2) $\mathscr{X}(\mathscr{A}(X)) \subseteq X$, and $\mathscr{X}(\mathscr{A}(X))=X$ if and only if $E_{\mathscr{A}(X)}(M)=E / X$.

Proof. (1) First compute,

$$
\mathscr{A}(\mathscr{X}(\mathscr{B}))=\mathscr{A}\left(\operatorname{ker} \pi_{\mathscr{B}}\right)=N \underset{E / \operatorname{ker} \pi_{\mathscr{B}}}{\square} M=N \underset{E_{\mathscr{B}}(M)}{\square} M .
$$

Now by virtue of Lemma 3.3.17 $\operatorname{ker} \pi_{\mathscr{B}} \subseteq \operatorname{ker} \pi_{\mathscr{A}} \subset \operatorname{ker} \pi$ and therefore $\pi: E \rightarrow D$ factors as $\pi: E \rightarrow E_{\mathscr{B}}(M) \rightarrow D$. Hence there is a coalgebra map $E_{\mathscr{B}}(M) \rightarrow D$ which induces an inclusion $N \underset{E_{\mathscr{B}}(M)}{\square} M \subseteq N \square$. In view of this enclosure we can draw the following diagram with exact rows.

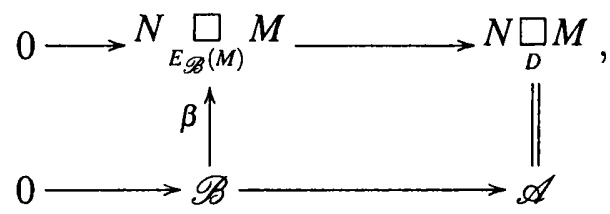

where $\beta$ is the map in Proposition 3.3.6 (with $\mathscr{B}$ in place of $\mathscr{A}$ ). Moreover for all $n \otimes m \in$ $\mathscr{B} \subseteq N \square_{D} M$ (summation suppressed),

$$
\begin{aligned}
\beta(n \otimes m) & =\sum n_{[-1]}^{[1]} \otimes \overline{\rho_{M}}\left(n_{[-1]}^{[2]} \otimes\left(n_{[0]} \otimes m\right)\right) \\
& =\sum n_{[-1]}^{[1]} \widehat{\tau}\left(n_{[-1]}^{[2]} \otimes n_{[0]}\right) \otimes m=n \otimes m,
\end{aligned}
$$

In the above for all $c \in C$ we have written $\sigma(c)=\sum c^{[1]} \otimes c^{[2]}$, where $\sigma$ is the counit of the $\mathscr{B}$-coendomorphism context, and $\widehat{\tau}$ is the reduced unit. The right $\mathscr{B}$-action on $M$ takes the above form since it is induced by the right $N \square_{D} M$ action on $M$. The final equality then follows by the equations (3.2). Therefore, the above diagram is commutative and the map $\beta$ is the required inclusion. Once the inclusion is described this way it is clear there will be equality if and only if $\beta$ is bijective, i.e. if $M$ is Galois $\mathscr{B}$-module.

(2) Since $\mathscr{X}(\mathscr{A}(X))=\operatorname{ker} \pi_{\mathscr{A}(X)}$ the first part of the statement follows by Lemma 3.3.17. The equality holds if and only if $\operatorname{ker} \pi_{\mathscr{A}(X)}=\operatorname{ker} \pi_{X}$, and this is equivalent to the statement that $E_{\mathscr{A}(X)}(M)=E / X$.

Remark 3.3.19. Since the diagram included in the previous proposition is commutative for all $C$-subrings $\mathscr{B} \subseteq \mathscr{A}$, it is valid in the case when $\mathscr{A}=\mathscr{B}$. In this situation it is then clear that the map $\beta$ is bijective and therefore $M$ must be a Galois $\mathscr{A}$-module. Since $D$ was an arbitrary choice of coalgebra for which there existed a coalgebra epimorphism $\pi: E \rightarrow D, M$ must be a Galois module for any associated matrix $C$-ring induced by a coalgebra epimorphism with domain $E$.

Corollary 3.3.20. The Galois connection constructed in Proposition 3.3.18 establishes a one-to-one correspondence between $C$-subrings $\mathscr{B} \subseteq \mathscr{A}$ such that $M$ is Galois $\mathscr{B}$-module and subcoideals $X \subseteq \operatorname{ker} \pi$ such that $E_{\mathscr{A}(X)}(M)=E / X$. 
Proof. For any subcoideal $X \subseteq \operatorname{ker} \pi$, the $C$-ring $\mathscr{A}(X)$ is the matrix $C$-ring induced by the projection $\pi_{X}: E \rightarrow E / X$, and therefore by the Remark 3.3.19M must be a Galois $\mathscr{A}(X)$-module. For the converse, observe that if $M$ is a Galois $\mathscr{B}$ module, then $\mathscr{X}(\mathscr{A}(\mathscr{X}(\mathscr{B})))=\mathscr{X}(\mathscr{B})$ by the first part of Proposition 3.3.18. Therefore $\mathscr{X}(\mathscr{B})$ is a subcoideal of ker $\pi$ satisfying the required property by the second part of Proposition 3.3.18.

Since every coideal in $X \subseteq$ ker $\pi$ induces an 'intermediate coextension' $E \rightarrow E / X \rightarrow D$, the Galois connection we have just constructed establishes a correspondence between these 'intermediate coextensions' and $C$-subrings $\mathscr{B} \subseteq \mathscr{A}$. From this standpoint the Galois connection described in this subsection can be understood as a dual version of the Galois connection for comatrix corings described in [20, Proposition 2.1]. The connection constructed in [20] generalizes a Galois connection that was given for Sweedler type corings in [33], which in turn, was a natural extension of the correspondence in Sweedler's Fundamental Theorem [37, Theorem 2.1].

\section{4 $C$-rings and invertible weak entwining structures}

We now look again at (invertible) weak entwining structures, this time from a $C$-ring theoretic point of view. We begin by showing how to associate a $C$-ring to a weak entwining structure. Then we present dual versions of the results for $A$-corings associated to weak entwining structures that we described in the second chapter. Furthermore we describe necessary and sufficient conditions conditions for a $C$-ring to be isomorphic to a $C$-ring which arises from a weak entwining structure. Throughout this section we shall use the notations for weak entwining structures that was introduce in Definitions 2.2.6 and 2.2.7, also since there is no scope for confusion we shall write $\varepsilon_{C}=\varepsilon$ and $\Delta_{C}=\Delta$.

Theorem 3.4.1. Let $\left(A, C, \psi_{R}\right)$ be a weak right-right entwining structure. Define the map

$$
\overline{p_{R}}: C \otimes A \rightarrow C \otimes A, \quad \overline{p_{R}}:=(C \otimes A \otimes \varepsilon) \circ\left(C \otimes \psi_{R}\right) \circ(\Delta \otimes A),
$$

and the set

$$
\mathscr{A}:=\operatorname{Im} \overline{p_{R}}=\left\{\sum_{\alpha, i} c_{(1)}^{i} \otimes a_{\alpha}^{i} \varepsilon\left(c_{(2)}^{i}\right) \mid \sum_{i} a^{i} \otimes c^{i} \in A \otimes C\right\} .
$$

Then $\overline{p_{R}}$ is a projection, i.e. $\overline{p_{R}} \circ \overline{p_{R}}=\overline{p_{R}}$, and

(1) $\mathscr{A}$ is a $(C, C)$-bicomodule with the left coaction ${ }^{\mathscr{A}} \rho:=\Delta \otimes A$ and the right coaction $\rho^{\mathscr{A}}:=\left(C \otimes \psi_{R}\right) \circ(\Delta \otimes A)$.

(2) The $(C, C)$-bicomodule $\mathscr{A}$ is a $C$-ring with product

$$
\mu_{\mathscr{A}}: \mathscr{A} \square{ }_{c} \mathscr{A} \rightarrow \mathscr{A}, \quad \sum_{i} c_{i} \otimes a_{i} \otimes c_{i}^{\prime} \otimes a_{i}^{\prime} \mapsto \sum_{i} c_{i} \otimes \varepsilon\left(c_{i}^{\prime}\right) a_{i} a_{i}^{\prime},
$$

and unit

$$
\eta_{\mathscr{A}}: C \rightarrow \mathscr{A}, \quad c \mapsto \overline{p_{R}}(c \otimes 1)
$$

(3) $\mathbf{M}_{\mathscr{A}} \equiv \mathbf{M}\left(\psi_{R}\right)_{A}^{C}$. 
Proof. Firstly observe that $\overline{p_{R}}$ is a projection since, for all $c \otimes a \in C \otimes A$,

$$
\begin{aligned}
& \overline{p_{R}} \circ \overline{p_{R}}(c \otimes a)=\sum_{\alpha} \overline{p_{R}}\left(c_{(1)} \otimes a_{\alpha} \varepsilon\left(c_{(2)}^{\alpha}\right)\right) \\
& =\sum_{\alpha, \beta} c_{(1)} \otimes a_{\alpha \beta} \varepsilon\left(c_{(2)}^{\beta}\right) \varepsilon\left(c_{(3)}^{\alpha}\right) \\
& =\sum_{\alpha} c_{(1)} \otimes a_{\alpha} \varepsilon\left(c_{(2)}{ }^{\alpha}{ }_{(1)}\right) \varepsilon\left(c_{(2)}{ }^{\alpha}{ }_{(2)}\right) \\
& =\sum_{\alpha} c_{(1)} \otimes a_{\alpha} \varepsilon\left(c_{(2)}^{\alpha}\right)=\overline{p_{R}}(c \otimes a) \text {. }
\end{aligned}
$$

Where the third equality follows by equation (2.18). That ${ }^{\mathscr{A}} \rho$ is a left coaction follows immediately from properties of the coalgebra $(C, \Delta, \varepsilon)$. To see that $\rho^{\mathscr{A}}$ defines a right $C$-coaction, first observe by using equation (2.18) we can compute, for all $a \in A$ and $c \in C$,

$$
\sum_{\alpha} \rho^{\mathscr{A}}\left(c_{(1)} \otimes a_{\alpha} \varepsilon\left(c_{(2)}^{\alpha}\right)\right)=\sum_{\alpha} c_{(1)} \otimes a_{\alpha} \otimes c_{(2)}^{\alpha}
$$

Having established this, we next show that $\rho^{\mathscr{A}} \circ \overline{p_{R}}=\left(\overline{p_{R}} \otimes C\right) \circ \rho^{\mathscr{A}} \circ \overline{p_{R}}$, since $\overline{p_{R}}$ is a projection, this will imply that $\rho^{\mathscr{A}} \subseteq \mathscr{A} \otimes C$. To see that this equality holds apply $\overline{p_{R}} \otimes C$ to equation (3.14) and observe

$$
\begin{aligned}
\sum_{\alpha}\left(\overline{p_{R}} \otimes C\right) \circ \rho^{\mathscr{A}} \circ \overline{p_{R}}(c \otimes a) & =\sum_{\alpha, \beta} c_{(1)} \otimes a_{\alpha \beta} \varepsilon\left(c_{(2)}^{\beta}\right) \otimes c_{(3)}^{\alpha} \\
& =\sum_{\alpha} c_{(1)} \otimes a_{\alpha} \otimes c_{(2)}^{\alpha}=\rho^{\mathscr{A}} \circ \overline{p_{R}}(c \otimes a) .
\end{aligned}
$$

Where the second equality follows by (2.18) and the the final equality by (3.14). Applying $C \otimes A \otimes \varepsilon$ to (3.14) it is immediate that $\rho^{\mathscr{A}}$ is counital. It now only remains to show that $\rho^{\mathscr{A}}$ is coassociative. Take any $c \otimes a \in C \otimes A$ (summation suppressed) and compute

$$
\begin{gathered}
\left(\rho^{\mathscr{A}} \otimes C\right) \circ \rho^{\mathscr{A}} \circ \overline{p_{R}}(c \otimes a)=\sum_{\alpha} \rho^{\mathscr{A}}\left(c_{(1)} \otimes a_{\alpha}\right) \otimes c_{(2)}{ }^{\alpha}=\sum_{\alpha, \beta} c_{(1)} \otimes a_{\alpha \beta} \otimes c_{(2)} \beta \otimes c_{(3)}{ }^{\alpha} \\
=\sum_{\alpha} c_{(1)} \otimes a_{\alpha} \otimes c_{(2)}{ }^{\alpha}{ }_{(1)} \otimes c_{(2)}{ }^{\alpha}{ }_{(2)}=(\mathscr{A} \otimes \Delta) \circ \rho^{\mathscr{A}} \circ \overline{p_{R}}(c \otimes a),
\end{gathered}
$$

where the the first and last equalities follow from (3.14), and the third equality follows by (2.18). That $\rho^{\mathscr{A}}$ and $\mathscr{A} \rho$ define a $C$-bicomodule structure on $\mathscr{A}$ is easily checked using the coassociativity of the coproduct $\Delta$ of $C$.

(2) First we need to check that $\mu_{\mathscr{A}}$ is well defined, i.e. $\mu_{\mathscr{A}}\left(\mathscr{A} \square_{C} \mathscr{A}\right) \subseteq \mathscr{A}$. Before attempting this calculation first observe that

$$
\mathscr{A} \square \mathscr{C}=\left\{\sum_{i, \alpha, \beta} c_{i(1)} \otimes a_{i \alpha} \otimes c_{i(2)}^{\alpha} \otimes a_{i \beta}^{\prime} \varepsilon\left(c_{i}^{\prime \beta}\right) \mid a_{i}, a_{i}^{\prime} \in A \text { and } c_{i}, c_{i}^{\prime} \in C\right\}
$$

This can be seen by applying $(C \otimes A \otimes C \otimes \varepsilon \otimes A)$ to the defining relation of the cotensor 
product $\mathscr{A} \square_{c} \mathscr{A}$. Now take any $a, a^{\prime} \in A$ and $c, c^{\prime} \in C$ and compute

$$
\begin{aligned}
\sum_{\alpha, \beta} \overline{p_{R}} \circ \mu_{\mathscr{A}}\left(c_{(1)} \otimes a_{\alpha} \otimes c_{(2)}{ }^{\alpha} \otimes a_{\beta}^{\prime}\right. & \left.\varepsilon\left(c^{\prime \beta}\right)\right) \\
& =\sum_{\alpha, \beta} \overline{p_{R}}\left(c_{(1)} \varepsilon\left(c_{(2)}^{\alpha}\right) \otimes a_{\alpha} a_{\beta}^{\prime} \varepsilon\left(c^{\prime \beta}\right)\right) \\
& =\sum_{\alpha, \beta, \gamma} c_{(1)} \varepsilon\left(c_{(3)}^{\alpha}\right) \otimes\left(a_{\alpha} a_{\beta}^{\prime}\right){ }_{\gamma} \varepsilon\left(c_{(2)}^{\gamma}\right) \varepsilon\left(c^{\prime \beta}\right) \\
& =\sum_{\alpha, \beta, \gamma, \omega} c_{(1)} \varepsilon\left(c_{(3)}^{\alpha}\right) \otimes a_{\alpha \gamma} a_{\beta \omega}^{\prime} \varepsilon\left(c_{(2)}^{\gamma \omega}\right) \varepsilon\left(c^{\prime \beta}\right) \\
& =\sum_{\alpha, \beta, \omega} c_{(1)} \otimes a_{\alpha} a_{\beta \omega}^{\prime} \varepsilon\left(c_{(2)}{ }^{\alpha \omega}\right) \varepsilon\left(c^{\prime \beta}\right) \\
& =\sum_{\alpha, \beta, \omega} c_{(1)} \otimes a_{\alpha} 1_{\omega} a_{\beta}^{\prime} \varepsilon\left(c_{(2)} \alpha \omega\right) \varepsilon\left(c^{\prime \beta}\right) \\
& =\sum_{\alpha, \beta} c_{(1)} \otimes a_{\alpha} a_{\beta}^{\prime} \varepsilon\left(c_{(2)}{ }^{\alpha}\right) \varepsilon\left(c^{\prime \beta}\right)
\end{aligned}
$$

Where the third and final equalities follow by (2.16), the fourth by (2.18) and the fifth by (2.17). Therefore since $\overline{p_{R}}$ is a projection we conclude that $\mu_{\mathscr{A}}\left(\mathscr{A} \square_{C} \mathscr{A}\right) \subseteq \mathscr{A}$. That $\mu_{\mathscr{A}}$ is left $C$-colinear is clear from its formulation. It is straight forward to check that $\mu_{\mathscr{A}}$ is right $C$-colinear by using (2.16). That $\mu_{\mathscr{A}}$ is associative is easily seen from its definition. It now remains to show that $\eta_{\mathscr{A}}$ is a $(C, C)$-bicolinear and that it is unit with respect to the product $\mu_{\mathscr{A}}$. From the definition of $\eta_{\mathscr{A}}$ it is clearly left $C$ colinear. Using (2.19) and (3.14) we immediately find

$$
\sum \overline{p_{R}}\left(c_{(1)} \otimes 1\right) \otimes c_{(2)}=\sum_{\alpha} c_{(1)} \otimes 1_{\alpha} \otimes c_{(2)}{ }^{\alpha}=\rho^{\mathscr{A}} \circ \overline{p_{R}}(1 \otimes c)
$$

hence $\eta_{\mathscr{A}}$ is right $C$-colinear as well. Making use of (3.14) and (2.16) it is easy to verify that $\mu_{\mathscr{A}} \circ\left(\mathscr{A} \square \eta_{C}\right)=\mathscr{A}$. Finally we compute, for all $a \in A$ and $c \in C$,

$$
\begin{aligned}
\mu_{\mathscr{A}} \circ\left(\eta_{\mathscr{A}} \square_{c} \mathscr{A}\right) \circ^{\mathscr{A}} \rho \circ \overline{p_{R}}(c \otimes a) & =\sum_{\alpha} \mu_{\mathscr{A}} \circ\left(\eta_{\mathscr{A}} \square_{c} \mathscr{A}\right)\left(c_{(1)} \otimes c_{(2)} \otimes a_{\alpha} \varepsilon\left(c_{(3)}^{\alpha}\right)\right) \\
& =\sum_{\alpha, \beta} c_{(1)} \otimes 1_{\beta} a_{\alpha} \varepsilon\left(c_{(2)}^{\beta}\right) \varepsilon\left(c_{(3)}^{\alpha}\right) \\
& =\sum_{\alpha, \beta} c_{(1)} \otimes a_{\alpha \beta} \varepsilon\left(c_{(2)}^{\beta}\right) \varepsilon\left(c_{(3)}{ }^{\alpha}\right) \\
& =\sum_{\alpha} c_{(1)} \otimes a_{\alpha} \varepsilon\left(c_{(2)}^{\alpha}\right)=\overline{p_{R}}(c \otimes a)
\end{aligned}
$$

where the third equality follows by (2.17). This completes the proof that the given maps define a $C$-ring structure on $\mathscr{A}$.

(3) First let $\Psi: \mathbf{M}_{\mathscr{A}} \rightarrow \mathbf{M}\left(\psi_{R}\right)_{A}^{C}$ be the map which leaves each $\mathscr{A}$-module unchanged as a $C$-comodule, but which changes the right $\mathscr{A}$-action $\overline{\rho_{M}}$ into a map

$$
\Psi\left(\overline{\rho_{M}}\right): M \otimes A \rightarrow M, \quad \Psi\left(\overline{\rho_{M}}\right):=\overline{\rho_{M}} \circ\left(M \square \bar{C} \overline{p_{R}}\right) \circ\left(\rho^{M} \otimes A\right) .
$$


We now show that each such map, $\Psi\left(\overline{\rho_{M}}\right)$, defines a right $A$-action on $M$ for which $M$ is an entwined module. Firstly to check the unitality, compute, for all $m \in M$,

$$
\Psi\left(\overline{\rho_{M}}\right)(m \otimes 1)=\sum \overline{\rho_{M}} \circ\left(M \square_{C} \overline{p_{R}}\right)\left(m_{[0]} \otimes m_{[1]} \otimes 1\right)=\overline{\rho_{M}} \circ\left(M \square_{C} \eta_{\mathscr{A}}\right) \circ \rho^{M}(m)=m,
$$

where the first equality uses the definition of the unit $\eta_{\mathscr{A}}$ and the second equality uses the unitality of the right $\mathscr{A}$-action. For the associativity, take any $a, a^{\prime} \in A$ and $m \in M$ and calculate

$$
\begin{aligned}
\Psi\left(\overline{\rho_{M}}\right) \circ\left(\Psi\left(\overline{\rho_{M}}\right) \otimes A\right)\left(m \otimes a \otimes a^{\prime}\right) & \\
& =\sum_{\alpha} \overline{\rho_{M}} \circ\left(M \square_{C} \overline{p_{R}}\right)\left(\rho^{M} \circ \overline{\rho_{M}}\left(m_{[0]} \otimes m_{[1]} \otimes a_{\alpha} \varepsilon\left(m_{[2]}{ }^{\alpha}\right)\right) \otimes a^{\prime}\right) \\
& =\sum_{\alpha} \overline{\rho_{M}} \circ\left(M \square_{C} \overline{p_{R}}\right)\left(\left(\overline{\rho_{M}} \otimes C\right)\left(m_{[0]} \otimes m_{[1]} \otimes a_{\alpha} \otimes m_{[2]}^{\alpha}\right) \otimes a^{\prime}\right) \\
& =\sum_{\alpha} \overline{\rho_{M}} \circ\left(\overline{\rho_{M}} \square_{C} \mathscr{A}\right)\left(m_{[0]} \otimes\left(m_{[1]} \otimes a_{\alpha}\right) \otimes \overline{p_{R}}\left(m_{[2]}^{\alpha} \otimes a^{\prime}\right)\right) \\
& =\sum_{\alpha} \overline{\rho_{M}} \circ\left(M \square_{C} \mu_{\mathscr{A}}\right)\left(m_{[0]} \otimes\left(m_{[1]} \otimes a_{\alpha}\right) \otimes \overline{p_{R}}\left(m_{[2]}^{\alpha} \otimes a^{\prime}\right)\right) \\
& =\sum_{\alpha, \beta} \overline{\rho_{M}}\left(m_{[0]} \otimes m_{[1]} \otimes a_{\alpha} a_{\beta}^{\prime} \varepsilon\left(m_{[2]}^{\alpha}{ }_{(1)}\right) \varepsilon\left(m_{[2]}^{\alpha}{ }_{(2)}^{\beta}\right)\right) \\
& =\sum_{\alpha} \overline{\rho_{M}}\left(m_{[0]} \otimes m_{[1]} \otimes\left(a a^{\prime}\right)_{\alpha} \varepsilon\left(m_{[2]}^{\alpha}\right)\right)=\Psi\left(\overline{\rho_{M}}\right)\left(m \otimes a a^{\prime}\right) .
\end{aligned}
$$

Where the second equality follows from the right $C$-colinearity of $\overline{\rho_{M}}$ and (3.14), and the fourth by the associativity of $\overline{\rho_{M}}$. Then the penultimate equality follows by the definition of a counit and (2.16). Now we proceed to show that this right action makes $M$ an entwined module

$$
\begin{aligned}
\left(\Psi\left(\overline{\rho_{M}}\right) \otimes C\right) \circ\left(M \otimes \psi_{R}\right) \circ\left(\rho^{M} \otimes A\right) & =\left(\overline{\rho_{M}} \otimes C\right) \circ\left(M \otimes C \otimes \psi_{R}\right) \circ(M \otimes \Delta \otimes A) \circ\left(\rho^{M} \otimes A\right) \\
& =\left(\overline{\rho_{M}} \otimes C\right) \circ\left(M \square_{C} \rho^{\mathscr{A}} \circ \overline{p_{R}}\right) \circ\left(\rho^{M} \otimes A\right) \\
& =\rho^{M} \circ \overline{\rho_{M}} \circ\left(M \square \overline{p_{R}}\right) \circ\left(\rho^{M} \otimes A\right)=\rho^{M} \circ \Psi\left(\overline{\rho_{M}}\right),
\end{aligned}
$$

where the first equality follows by the coassociativity of a coaction, the definition of a counit and (2.18), the second by (3.14) and penultimate equality by the colinearity of $\overline{\rho_{M}}$.

We have seen what $\Psi$ does to the objects of $\mathbf{M}_{\mathscr{A}}$, but we also need to know what it does to the morphisms. Given a morphism $f: M \rightarrow N$ in $\mathbf{M}_{\mathscr{A}}$, we define $\Psi(f)=f$. Using the right $C$-colinearity of $f$ and the the right $\mathscr{A}$-linearity of $f$, we can calculate that

$$
\begin{aligned}
f \circ \Psi\left(\overline{\rho_{M}}\right) & =\left(f \circ \overline{\rho_{M}}\right) \circ\left(M \square_{C} \overline{p_{R}}\right) \circ\left(\rho^{M} \otimes A\right) \\
& =\overline{\rho_{N}} \circ(f \square \mathscr{A}) \circ\left(M \square_{C} \overline{p_{R}}\right) \circ\left(\rho^{M} \otimes A\right) \\
& =\overline{\rho_{N}} \circ\left(N \square_{C} \overline{p_{R}}\right) \circ\left(\left(\rho^{N} \circ f\right) \otimes A\right) \\
& =\Psi\left(\overline{\rho_{N}}\right) \circ(f \otimes A) .
\end{aligned}
$$

Hence when $M$ and $N$ are viewed as $A$-modules with actions $\Psi\left(\overline{\rho_{M}}\right)$ and $\Psi\left(\overline{\rho_{N}}\right)$ respectively, the map $\Psi(f)$ is right $A$-linear. Therefore $\Psi$ is a functor. 
Conversely, define $\Theta: \mathbf{M}(\psi)_{A}^{C} \rightarrow \mathbf{M}_{\mathscr{A}}$ to be the map which leaves each entwined module $M$ unchanged as a $C$-comodule, but which changes the right $A$-action $\rho_{M}$ into a map

$$
\Theta\left(\rho_{M}\right): M \square_{C} \mathscr{A} \rightarrow M, \quad \Theta\left(\rho_{M}\right)=\rho_{M} \circ(M \otimes \varepsilon \otimes A) .
$$

To see that this gives an associative action, observe that all elements of $M \underset{c}{\square} \mathscr{A} \underset{c}{\square} \mathscr{A}$ are linear combinations of $x=\sum_{\alpha, \beta} m_{[0]} \otimes m_{[1]} \otimes a_{\alpha} \otimes m_{[2]}^{\alpha} \otimes a_{\beta}^{\prime} \varepsilon\left(c^{\beta}\right)$ with $a, a^{\prime} \in A$ and $m \in$ $M$. For such elements we can compute

$$
\begin{aligned}
\Theta\left(\rho_{M}\right) \circ(M \underset{c}{\square} \mu)(x) & =\sum_{\alpha, \beta} \Theta\left(\rho_{M}\right)\left(m_{[0]} \otimes m_{[1]} \varepsilon\left(m_{[2]}^{\alpha}\right) \otimes a_{\alpha} a_{\beta}^{\prime} \varepsilon\left(c^{\beta}\right)\right) \\
& =\sum_{\alpha, \beta} m_{[0]}\left(a_{\alpha} a_{\beta}^{\prime}\right) \varepsilon\left(m_{[1]}^{\alpha}\right) \varepsilon\left(c^{\beta}\right) \\
& =\sum_{\alpha, \beta}\left(m_{[0]} a_{\alpha}\right) a_{\beta}^{\prime} \varepsilon\left(m_{[1]}^{\alpha}\right) \varepsilon\left(c^{\beta}\right) \\
& =\Theta\left(\rho_{M}\right) \circ\left(\Theta\left(\rho_{M}\right) \underset{C}{\square} \mathscr{A}\right)(x) .
\end{aligned}
$$

Where the third equality follows by the associativity of $\rho_{M}$. Therefore since all maps are k-linear $\Theta\left(\rho_{M}\right)$ must be associative. Also observe, for all $m \in M$

$$
\Theta\left(\rho_{M}\right)\left(M \square_{c} \eta_{\mathscr{A}}\right)(m)=\sum_{\alpha} m_{[0]} 1_{\alpha} \varepsilon\left(m_{[1]}^{\alpha}\right)=m 1=m .
$$

Where the second equality follows since $M \in \mathbf{M}(\psi)_{A}^{C}$ and the final equality since $\rho_{M}$ is unital. Hence $\Theta\left(\rho_{M}\right)$ is unital. To show that $\Theta\left(\rho_{M}\right)$ gives a right $\mathscr{A}$-action on $M$ we need to check that it is a right $C$-colinear map. Since $M \square_{C} \mathscr{A}=\left(M \square_{C} \overline{p_{R}}\right)\left(M \square_{C} C \otimes A\right)$, all elements of $M \square_{C} \mathscr{A}$ are linear combinations of $y=\sum_{\alpha} m_{[0]} \otimes m_{[1]} \otimes a_{\alpha} \varepsilon\left(m_{[2]}^{\alpha}\right)$ with $a \in A$ and $m \in M$. Then using (3.14) and the fact $M \in \mathbf{M}(\psi)_{A}^{C}$, we can compute

$$
\begin{aligned}
\rho^{M} \circ \Theta\left(\rho_{M}\right)(y) & =\sum_{\alpha} m_{[0]} a_{\alpha} \otimes m_{[1]}^{\alpha}=\left(\rho_{M} \otimes C\right) \circ(M \otimes \varepsilon \otimes A \otimes C) \circ\left(M \otimes \rho^{\mathscr{A}}\right)(y) \\
& =\left(\Theta\left(\rho_{M}\right) \otimes C\right) \circ\left(M \otimes \rho^{\mathscr{A}}\right)(y) .
\end{aligned}
$$

Since all maps are assumed to be $k$-linear this shows that $\Theta\left(\rho_{M}\right)$ is a right $C$-colinear map.

Given a morphism $f: M \rightarrow N$ in $\mathbf{M}(\psi)_{A}^{C}$, define $\Theta(f)=f$. Clearly $\Theta(f)$ is always right $C$-colinear. Moreover in view of the definition of the $\mathscr{A}$-action and the $A$-linearity of $f$, it is easily checked that $\Theta(f)$ is right $\mathscr{A}$-linear. Since the composition in both categories is provided by the composition in the category of vector spaces, $\Theta: \mathbf{M}(\psi)_{A}^{C} \rightarrow \mathbf{M}_{\mathscr{A}}$ is a functor.

Finally, to complete the proof we need to demonstrate that $\Psi$ and $\Theta$ are inverse isomorphisms of the categories. Firstly for all $M \in \mathbf{M}(\psi)_{A}^{C}, m \in M$ and $a \in A$,

$$
\Psi\left(\Theta\left(\rho_{M}\right)\right)(m \otimes a)=\sum_{\alpha} \rho_{M}\left(m_{[0]} \otimes a_{\alpha} \varepsilon\left(m_{[1]}^{\alpha}\right)\right)=\sum(m a)_{[0]} \varepsilon\left((m a)_{[1]}\right)=\rho_{M}(m \otimes a),
$$

where the second equality follows by the fact that $M$ is an entwined module. On the other hand, taking $M \in \mathbf{M}_{\mathscr{A}}$ and applying $\left(\Theta\left(\Psi\left(\overline{\rho_{M}}\right)\right)\right.$ to $y=\sum_{\alpha} m_{[0]} \otimes m_{[1]} \otimes a_{\alpha} \varepsilon\left(m_{[2]}{ }^{\alpha}\right)$ we immediately obtain that $\left(\Theta\left(\Psi\left(\overline{\rho_{M}}\right)\right)(y)=\overline{\rho_{M}}(y)\right.$, completing the proof. 
Definition 3.4.2. Let $A$ be an algebra and $C$ be a coalgebra that is also a right $A$-module. Then the space

$$
I:=\operatorname{span}\left\{\sum(c a)_{(1)} \alpha\left((c a)_{(2)}\right)-\sum c_{(1)} \alpha\left(c_{(2)} a\right) \mid a \in A, c \in C, \alpha \in \operatorname{Hom}_{k}(C, k)\right\}
$$

is a coideal of $C$ (see [9, Lemma 3.2] for a proof of this). Now $B:=C / I$ is a coalgebra and we say $C \rightarrow B$ is a (right) weak algebra-Galois coextension (or a weak A-Galois coextension) provided that the left $A$-linear right $C$-colinear map

$$
\beta: C \otimes A \rightarrow C \square_{B} C, \quad \beta:=\left(C \otimes \rho_{C}\right) \circ(\Delta \otimes A)
$$

is a split epimorphism in the category ${ }^{C} \mathbf{M}_{A}$, that is to say there exists a morphism $\bar{\chi}$ : $C \square_{B} C \rightarrow C \otimes A$ in ${ }^{C} \mathbf{M}_{A}$ such that $\beta \circ \bar{\chi}=C \square_{B} C$.

In the weak algebra-Galois coextension case there is a result dual to Theorem 2.2.12.

Theorem 3.4.3. Let $C \rightarrow B$ be a weak A-Galois coextension. Then there exists a unique right-right weak entwining structure $\left(A, C, \psi_{R}\right)$ such that $C \in \mathbf{M}\left(\psi_{R}\right)_{A}^{C}$ with the canonical right $C$-coaction (via the coproduct in $C$ ) and the predetermined right $A$-action. This weak entwining structure is referred to as the canonical weak entwining structure associated to the A-Galois coextension $C \rightarrow B$.

Proof. Let $\bar{\chi}$ denote the map which splits $\beta$ and set

$$
\tilde{\tau}: C \square_{B} C \rightarrow A, \quad \tilde{\tau}:=(\varepsilon \otimes A) \circ \bar{\chi},
$$

to be what will refer to as the weak cotranslation map. We first investigate some properties enjoyed by such a map. Firstly

$$
\begin{aligned}
\rho_{C} \circ(C \otimes \tilde{\tau}) \circ(\Delta \otimes C) & =\rho_{C} \circ(C \otimes \varepsilon \otimes A) \circ(C \otimes \bar{\chi}) \circ(\Delta \otimes C) \\
& =\rho_{C} \circ(C \otimes \varepsilon \otimes A) \circ(\Delta \otimes A) \circ \bar{\chi} \\
& =\rho_{C} \circ \bar{\chi} \\
& =(\varepsilon \otimes C) \circ \beta \circ \bar{\chi},
\end{aligned}
$$

where the second equality follows from the left $C$-colinearity of $\bar{\chi}$ and the last equality by noting that $(\varepsilon \otimes C) \circ \beta(c \otimes a)=\sum \varepsilon\left(c_{(1)}\right) \otimes c_{(2)} a=c a$. Now since $\bar{\chi}$ splits $\beta$ this implies

$$
\rho_{C} \circ(C \otimes \tilde{\tau}) \circ(\Delta \otimes C)=\varepsilon \otimes C .
$$

Also observe that

$$
\begin{aligned}
\tilde{\tau} \circ\left(C \otimes \rho_{C}\right)\left(c \otimes c^{\prime} \otimes a\right) & =(\varepsilon \otimes A) \circ \bar{\chi}\left(c \otimes c^{\prime} a\right) \\
& =(\varepsilon \otimes A) \circ \bar{\chi}\left(c \otimes c^{\prime}\right) a \\
& =\tilde{\tau}\left(c \otimes c^{\prime}\right) a \\
& =\mu \circ(\tilde{\tau} \otimes A) \circ\left(c \otimes c^{\prime} \otimes a\right)
\end{aligned}
$$

where the right $A$-action on $C \otimes A$ is $C \otimes \mu$ and the second equality follows by the right $A$-linearity of $\bar{\chi}$. From this observation it is then clear that

$$
\mu \circ(\tilde{\tau} \otimes \tilde{\tau})=\tilde{\tau} \circ\left(C \otimes \rho_{C} \circ(C \otimes \tilde{\tau})\right),
$$


on $C \square_{B} C \otimes C \square_{B} C$. Combining the previous equation, (3.19), with equation (3.18) it is clear that on $C \square_{B} C \square_{B}^{B} C$ we have

$$
\mu \circ(\tilde{\tau} \otimes \tilde{\tau}) \circ(C \otimes \Delta \otimes C)=\tilde{\tau} \circ(C \otimes \varepsilon \otimes C) .
$$

Making use of the above observations and the elementary properties of the map in questions, we now show that the map

$$
\psi_{R}: C \otimes A \rightarrow A \otimes C, \quad \psi_{R}:=(\tilde{\tau} \otimes C) \circ(C \otimes \Delta) \circ \beta,
$$

gives a right-right weak entwining structure $\left(A, C, \psi_{R}\right)$. Firstly that

$$
(A \otimes \varepsilon \otimes C) \circ\left(\psi_{R} \otimes C\right) \circ(C \otimes 1 \otimes C) \circ \Delta=\psi_{R} \circ(C \otimes 1)
$$

and

$$
\mu \circ(A \otimes \varepsilon \otimes A) \circ\left(\psi_{R} \otimes A\right) \circ(C \otimes 1 \otimes A)=(A \otimes \varepsilon) \circ \psi_{R}
$$

can easily be confirmed by a straight forward calculation. Next compute

$$
\begin{aligned}
(\mu \otimes C) \circ(A & \left.\otimes \psi_{R}\right) \circ\left(\psi_{R} \otimes A\right) \\
& =((\mu \circ(\tilde{\tau} \otimes \tilde{\tau})) \otimes C) \circ\left(C^{\otimes 3} \otimes \Delta\right) \circ(C \otimes((C \otimes \beta) \circ(\Delta \otimes A))) \circ(\beta \otimes A) \\
& =((\mu \circ(\tilde{\tau} \otimes \tilde{\tau})) \otimes C) \circ\left(C^{\otimes 3} \otimes \Delta\right) \circ(C \otimes((\Delta \otimes C) \circ \beta)) \circ(\beta \otimes A) \\
& =((\mu \circ(\tilde{\tau} \otimes \tilde{\tau}) \circ(C \otimes \Delta \otimes C) \otimes C) \circ(C \otimes C \otimes \Delta) \circ(C \otimes \beta) \circ(\beta \otimes A) \\
& =((\widetilde{\tau} \circ(C \otimes \varepsilon \otimes C)) \otimes C) \circ(C \otimes C \otimes \Delta) \circ(C \otimes \beta) \circ(\beta \otimes A) \\
& =(\tilde{\tau} \otimes C) \circ(C \otimes((\varepsilon \otimes C \otimes C) \circ(C \otimes \Delta) \circ \beta)) \circ(\beta \otimes A) \\
& =(\tilde{\tau} \otimes C) \circ(C \otimes \Delta) \circ\left(C \otimes \rho_{C}\right) \circ(\beta \otimes A) \\
& =(\tilde{\tau} \otimes C) \circ(C \otimes \Delta) \circ \beta \circ(C \otimes \mu) \\
& =\psi_{R} \circ(C \otimes \mu) .
\end{aligned}
$$

Here the second equality follows by the left $C$-colinearity of $\beta$ and the fourth by equation (3.20). For the sixth equality observe that

$$
(\varepsilon \otimes C \otimes C) \circ(C \otimes \Delta) \circ \beta(c \otimes a)=\sum \varepsilon\left(c_{(1)}\right) \Delta\left(c_{(2)} a\right)=\Delta(c a) .
$$

Finally the penultimate equality follows from the right $A$-linearity of $\beta$. Now there is only one thing left to check in order to demonstrate that $\left(A, C, \psi_{R}\right)$ is a weak right-right entwining structure. Again compute

$$
\begin{aligned}
\left(\psi_{R} \otimes C\right) \circ & \left(C \otimes \psi_{R}\right) \circ(\Delta \otimes A) \\
& =\left(\psi_{R} \otimes C\right) \circ(C \otimes \tilde{\tau} \otimes C) \circ(C \otimes C \otimes \Delta) \circ(C \otimes \beta) \circ(\Delta \otimes A) \\
& =\left(\psi_{R} \otimes C\right) \circ(C \otimes \tilde{\tau} \otimes C) \circ(C \otimes C \otimes \Delta) \circ(\Delta \otimes C) \circ \beta \\
& =(\tilde{\tau} \otimes C \otimes C) \circ(C \otimes \Delta \otimes C) \circ((\beta \circ(C \otimes \tilde{\tau}) \circ(\Delta \otimes C)) \otimes C) \circ(C \otimes \Delta) \circ \beta \\
& =(\tilde{\tau} \otimes C \otimes C) \circ(C \otimes \Delta \otimes C) \circ((\beta \circ \bar{\chi}) \otimes C) \circ(C \otimes \Delta) \circ \beta \\
& =(\tilde{\tau} \otimes C \otimes C) \circ(C \otimes C \otimes \Delta) \circ(C \otimes \Delta) \circ \beta \\
& =(A \otimes \Delta) \circ(\tilde{\tau} \otimes C) \circ(C \otimes \Delta) \circ \beta \\
& =(A \otimes \Delta) \circ \psi_{R} .
\end{aligned}
$$


Here the second equality follows by the left $C$-colinearity of $\beta$ and the fourth follows because using the colinearity of $\chi$

$$
(C \otimes \widetilde{\tau}) \circ(\Delta \otimes C)=(C \otimes \varepsilon \otimes A) \circ(C \otimes \bar{\chi}) \circ(\Delta \otimes C)=(C \otimes \varepsilon \otimes A) \circ(\Delta \otimes A) \circ \bar{\chi}=\bar{\chi} .
$$

Finally, the sixth equality in the computation of $\left(\psi_{R} \otimes C\right) \circ\left(C \otimes \psi_{R}\right) \circ(\Delta \otimes A)$ follows by the coassociativity of $\Delta$. Hence $\left(A, C, \psi_{R}\right)$ is a weak right-right entwining structure. Now we can follow the same proof as in [9, Theorem 3.5], to show that $C \in \mathbf{M}(\psi)_{A}^{C}$ with the canonical action and coaction:

$$
\begin{aligned}
\left(\rho_{C} \otimes C\right) \circ\left(C \otimes \psi_{R}\right) \circ(\Delta & \otimes A) \\
& =\left(\rho_{C} \otimes C\right) \circ(C \otimes \tilde{\tau} \otimes C) \circ(C \otimes C \otimes \Delta) \circ(C \otimes \beta) \circ(\Delta \otimes A) \\
& =\left(\left(\rho_{C} \circ(C \otimes \tilde{\tau})\right) \otimes C\right) \circ(C \otimes C \otimes \Delta) \circ(\Delta \otimes C) \circ \beta \\
& =\left(\left(\rho_{C} \circ(C \otimes \tilde{\tau}) \circ(\Delta \otimes C)\right) \otimes C\right) \circ(C \otimes \Delta) \circ \beta \\
& =(\varepsilon \otimes C \otimes C) \circ(C \otimes \Delta) \circ \beta \\
& =\Delta \circ \rho_{C} .
\end{aligned}
$$

In this computation the second equality follows by the left $C$-colinearity of $\beta$, the fourth by equation (3.18) and the final equality by equation (3.21). Finally to complete the proof of the theorem the uniqueness of $\psi_{R}$ can be demonstrated as follows. Suppose there exists some other weak entwining map $\tilde{\psi}_{R}$ such that $C \in \mathbf{M}\left(\tilde{\psi}_{R}\right)_{A}^{C}$ with the obvious action and coaction. Then

$$
\begin{aligned}
\psi_{R} & =(\tilde{\tau} \otimes C) \circ(C \otimes \Delta) \circ\left(C \otimes \rho_{C}\right) \circ(\Delta \otimes A) \\
& =(\tilde{\tau} \otimes C) \circ\left(C \otimes\left(\left(\rho_{C} \otimes C\right) \circ\left(C \otimes \tilde{\psi}_{R}\right) \circ(\Delta \otimes A)\right)\right) \circ(\Delta \otimes A) \\
& =\left(\left(\tilde{\tau} \circ\left(C \otimes \rho_{C}\right)\right) \otimes C\right) \circ\left(C \otimes C \otimes \tilde{\psi}_{R}\right) \circ(C \otimes \Delta \otimes A) \circ(\Delta \otimes A) \\
& =\left(\left(\tilde{\tau} \circ\left(C \otimes \rho_{C}\right)\right) \otimes C\right) \circ\left(\Delta \otimes \tilde{\psi}_{R}\right) \circ(\Delta \otimes A) \\
& =\left(\left(\tilde{\tau} \circ\left(C \otimes \rho_{C}\right) \circ(\Delta \otimes A)\right) \otimes C\right) \circ\left(C \otimes \tilde{\psi}_{R}\right) \circ(\Delta \otimes A) \\
& =((\tilde{\tau} \circ \beta) \otimes C) \circ\left(C \otimes \tilde{\psi}_{R}\right) \circ(\Delta \otimes A) \\
& =(\varepsilon \otimes A \otimes C) \circ\left(C \otimes \tilde{\psi}_{R}\right) \circ(\Delta \otimes A)=\tilde{\psi}_{R} .
\end{aligned}
$$

Here the second equality follows by the compatibility condition required so that $C \in$ $\mathbf{M}\left(\tilde{\psi}_{R}\right)_{A}^{C}$ with the prescribed action and coaction. The fourth equality follows by the coassociativity of $\Delta$. Finally the sixth and seventh equalities follow from the definitions of the maps $\beta$ and $\tilde{\tau}$ respectively.

Having established how to associate a weak entwining structure to a weak algebraGalois coextension, we now describe a necessary and sufficient condition on a weak entwining structure to determine whether it arises from a weak algebra-Galois coextension. Recall that in the case of weak coalgebra-Galois extensions (c.f. Theorem 2.2.12) this can be achieved by looking at the associated $A$-coring, as described in Proposition 2.2.14.

Lemma 3.4.4. Suppose $C \in \mathbf{M}\left(\psi_{R}\right)_{A}^{C}$ with the natural right $C$-coaction. Then the coideal I of $C$, described in Definition 3.4.2, coincides with the coideal $I_{\kappa}$ defined in Lemma 3.3.10. Where $\kappa: \mathscr{A} \rightarrow k$ is the nontrivial character associated to the right action on $C$ of the $C$-ring $\mathscr{A}$ arising from $\left(A, C, \psi_{R}\right)$ (cf. Lemma 3.3.9 and Theorem 3.4.1). 
Proof. First observe that by equations (3.9) and (3.17) we can compute that, for all $c \otimes a \in \mathscr{A}$,

$$
\begin{aligned}
\kappa(c \otimes a) & =\varepsilon \circ \rho_{C} \circ(C \otimes \varepsilon \otimes A) \circ \mathscr{A} \rho(c \otimes a) \\
& =\sum \varepsilon \circ \rho_{C} \circ(C \otimes \varepsilon \otimes A)\left(c_{(1)} \otimes c_{(2)} \otimes a\right)=\varepsilon(c a) .
\end{aligned}
$$

In view of this observation it is clear that

$$
I_{\kappa}=\left\{c a-\sum c_{(1)} \varepsilon\left(c_{(2)} a\right) \mid c \otimes a \in \mathscr{A}\right\}
$$

and since $\varepsilon \in \operatorname{Hom}_{k}(C, k)$, it is clear that $I_{\kappa} \subseteq I$. On the other hand all elements of $I$ are linear combination of $x=\sum(c a)_{(1)} \xi\left((c a)_{(2)}\right)-\sum c_{(1)} \xi\left(c_{(2)} a\right)$ with $a \in A, c \in C$ and $\xi \in \operatorname{Hom}_{k}(C, k)$. Now observe that for each such $x$

$$
\begin{aligned}
x & =\sum_{\alpha} c_{(1)} a_{\alpha} \xi\left(c_{(2)}^{\alpha}\right)-c_{(1)} \varepsilon\left(\left(c_{(2)} a\right)_{(1)}\right) \xi\left(\left(c_{(2)} a\right)_{(2)}\right) \\
& =\sum_{\beta} c_{(1)} a_{\beta} \xi\left(c_{(2)}^{\beta}\right)-\sum_{\alpha} c_{(1)} \varepsilon\left(c_{(2)} a_{\alpha}\right) \xi\left(c_{(3)}^{\alpha}\right) \\
& =\sum_{\alpha, \beta} c_{(1)} a_{\alpha \beta} \varepsilon\left(c_{(2)}^{\beta}\right) \xi\left(c_{(3)}^{\alpha}\right)-c_{(1)} \varepsilon\left(c_{(2)} a_{\alpha}\right) \xi\left(c_{(3)}^{\alpha}\right) .
\end{aligned}
$$

Here the first and second equalities follow since $C \in \mathbf{M}\left(\psi_{R}\right)_{A}^{C}$ with the canonical right $C$ coaction, and the third by equation (2.18). Using (2.18) it is straight forward to verify that $\Sigma_{\alpha} c_{(1)} \otimes a_{\alpha} \xi\left(c_{(2)}{ }^{\alpha}\right) \in \mathscr{A}$. Hence we can conclude that $x \in I_{\kappa}$ and therefore that $I \subseteq I_{\kappa}$.

Lemma 3.4.5. Suppose $\mathscr{A}$ is a $C$-ring associated to a weak entwining structure $\left(\psi_{R}, A, C\right)$ such that $C \in \mathbf{M}_{\mathscr{A}}=\mathbf{M}\left(\psi_{R}\right)_{A}^{C}$. Then $\mathscr{A}$ is a Galois $C$-ring (with respect to $\kappa=\varepsilon \circ \rho_{C}$ ) if and only if $\left.\bar{\beta}\right|_{\mathscr{A}}: \mathscr{A} \rightarrow C \underset{B}{ } C$ is a bijection.

Proof. Observe that for this choice of $\kappa$ the induced $C$-ring map $\Gamma_{\kappa}: \mathscr{A} \rightarrow C \square_{B_{\kappa}} C$, as described in Proposition3.3.12, satisfies, for all $c \otimes a \in \mathscr{A}$,

$$
\begin{aligned}
\Gamma_{\kappa}(c \otimes a) & =\sum_{\alpha} c_{(1)} \otimes \varepsilon\left(c_{(2)} a_{\alpha}\right) c_{(3)}^{\alpha} \\
& =\sum c_{(1)} \otimes c_{(2)} a=\beta(c \otimes a) .
\end{aligned}
$$

Where the first equality follows since $C \in \mathbf{M}\left(\psi_{R}\right)_{A}^{C}$. Also in view of the previous lemma $B_{\kappa}=B . \Gamma_{\kappa}: \mathscr{A} \rightarrow C \square_{B_{K}} C$ is an isomorphism if and only if $\left.\beta\right|_{\mathscr{A}}: \mathscr{A} \rightarrow C \square_{B} C$ is a bijection.

Proposition 3.4.6. The $C$-ring associated to the weak entwining structure in Theorem 3.4.3 is a Galois $C$-ring. Conversely, if the $C$-ring associated to a weak entwining structure $\left(A, C, \psi_{R}\right)$ is a Galois $C$-ring, then $C$ is a weak algebra-Galois coextension. 
Proof. If $\mathscr{A}$ is the $C$-ring associated to the weak entwining structure in Theorem 3.4.3, then for the associated projection $\overline{p_{R}}: C \otimes A \rightarrow C \otimes A$,

$$
\begin{aligned}
\overline{p_{R}} & =(C \otimes A \otimes \varepsilon) \circ\left(C \otimes \psi_{R}\right) \circ(\Delta \otimes A) \\
& =(C \otimes A \otimes \varepsilon) \circ(C \otimes \varepsilon \otimes A \otimes C) \circ(C \otimes \bar{\chi} \otimes C) \circ(C \otimes C \otimes \Delta) \circ(C \otimes \beta) \circ(\Delta \otimes A) \\
& =(C \otimes \varepsilon \otimes A) \circ(C \otimes \bar{\chi}) \circ(C \otimes \beta) \circ(\Delta \otimes A) \\
& =(C \otimes \varepsilon \otimes A) \circ(C \otimes \bar{\chi}) \circ(\Delta \otimes C) \circ \beta \\
& =(C \otimes \varepsilon \otimes A) \circ(\Delta \otimes A) \circ \bar{\chi} \circ \beta=\bar{\chi} \circ \beta,
\end{aligned}
$$

where the fourth and fifth equalities follow by the left $C$-colinearity of $\beta$ and $\bar{\chi}$ respectively. Hence $\mathscr{A}=\operatorname{Im}(\bar{\chi} \circ \bar{\beta})$ and since $\bar{\beta} \circ \bar{\chi}=C \square_{B} C$, the map $\left.\bar{\beta}\right|_{\mathscr{A}}$ is a bijection. Therefore, by Lemma 3.4.5, $\mathscr{A}$ is a Galois $C$-ring. Conversely if $\mathscr{A}$ is a Galois $C$-ring and associated to a weak entwining structure then by Lemma 3.4.5, $\left.\bar{\beta}\right|_{\mathscr{A}}: \mathscr{A} \rightarrow C \square_{B} C$ is a bijection, furthermore it is clear from the definition of $\bar{\beta}$ that it is a morphism in ${ }^{C} \mathbf{M}_{A}$. Now observe that the composition of the maps $\left.\bar{\beta}\right|_{\mathscr{A}} ^{-1}: C \square_{B} C \rightarrow \mathscr{A}$ and then the inclusion $\mathscr{A} \hookrightarrow C \otimes A$ is a morphism in ${ }^{C} \mathbf{M}_{A}$ splitting $\bar{\beta}$. Therefore $C \rightarrow B$ is a weak algebra-Galois coextension.

We have already demonstrated in this section how it is possible to associate a $C$-ring to a right-right entwining structure, now we show how to associate a $C$-ring to a left-left weak entwining structure.

Theorem 3.4.7. Let $\left(A, C, \psi_{L}\right)$ be a left-left weak entwining structure. Define the map

$$
\overline{p_{L}}: A \otimes C \rightarrow A \otimes C, \quad \overline{p_{L}}:=(\varepsilon \otimes A \otimes C) \circ\left(\psi_{L} \otimes C\right) \circ(A \otimes \Delta),
$$

and the set

$$
\mathscr{B}:=\operatorname{Im} \overline{p_{L}}=\left\{\sum_{E, i} \varepsilon\left(c^{i}{ }_{(1) E}\right) a^{i E} \otimes c_{(2)} \mid \sum_{i} a^{i} \otimes c^{i} \in A \otimes C\right\} .
$$

Then $\overline{p_{L}}$ is a projection, i.e. $\overline{p_{L}} \circ \overline{p_{L}}=\overline{p_{L}}$, and

(1) $\mathscr{B}$ is a $(C, C)$-bicomodule with the left coaction ${ }^{\mathscr{B}} \rho:=\left(A \otimes \psi_{L}\right) \circ\left(A \otimes \Delta_{C}\right)$ and the right coaction $\rho^{\mathscr{B}}:=A \otimes \Delta_{C}$.

(2) The $(C, C)$-bicomodule $\mathscr{B}$ is a $C$-ring with product

$$
\mu_{\mathscr{B}}: \mathscr{B} \square_{C} \mathscr{B} \rightarrow \mathscr{B}, \quad \sum_{i} a_{i} \otimes c_{i} \otimes a_{i}^{\prime} \otimes c_{i}^{\prime} \mapsto \sum_{i} a_{i} a_{i}^{\prime} \varepsilon\left(c_{i}\right) \otimes c_{i}^{\prime},
$$

and unit

$$
\eta_{\mathscr{B}}: C \rightarrow \mathscr{B}, \quad c \mapsto \overline{p_{L}}(1 \otimes c)
$$

(3) $\mathscr{B} \mathbf{M} \equiv{ }_{A}^{C} \mathbf{M}\left(\psi_{L}\right)$.

Proof. This theorem is simply a 'left handed' version of Theorem 3.4.1, and can be proved in a similar manner.

Before investigating the $C$-rings associated to an invertible weak entwining structure, it will be useful to recall the following result [1, Proposition 1.5]. 
Lemma 3.4.8. Let $\left(A, C, \psi_{R}, \psi_{L}\right)$ be an invertible weak entwining structure. Then $\overline{p_{R}}=p_{L}$ and $\overline{p_{L}}=p_{R}$.

Proof. To see that $\overline{p_{R}}=p_{L}$, take any $a \in A$ and $c \in C$, and compute

$$
\begin{aligned}
\overline{p_{R}}(c \otimes a) & =\sum_{\alpha} c_{(1)} \otimes a_{\alpha} \varepsilon\left(c_{(2)}^{\alpha}\right)=\sum_{\alpha} c_{(1)} \otimes \varepsilon\left(c_{(2)}^{\alpha}\right) 1_{\alpha} a \\
& =\sum_{E} c_{(1)} \otimes \varepsilon\left(c_{(2)_{E}}\right) 1^{E} a=\sum_{E} c_{E} \otimes 1^{E} a=p_{L}(c \otimes a),
\end{aligned}
$$

where the second equality follows by (2.17), the third by (2.24), and the fourth by (2.23). A similar calculation shows that $\overline{p_{L}}=p_{R}$.

Remark 3.4.9. Lemma 3.4.8 shows that conditions (b) in the definition of an invertible weak entwining structure (Definition 2.3.2) may be replaced with alternative conditions:

(b*) $\psi_{R} \circ \psi_{L}=\overline{p_{L}}$ and $\psi_{L} \circ \psi_{R}=\overline{p_{R}}$.

Note further that both $\mathscr{A}$ and $\mathscr{B}$ are not only $C$-rings but also $A$-corings.

Remark 3.4.10. Using the previous remark and (2.18), it is clear that

$$
\psi_{R} \circ \overline{p_{R}}=p_{R} \circ \psi_{R}=\psi_{R}
$$

Similarly using (2.22)

$$
\psi_{L} \circ \overline{p_{L}}=p_{L} \circ \psi_{L}=\psi_{L}
$$

Proposition 3.4.11. Let $\left(A, C, \psi_{R}, \psi_{L}\right)$ be an invertible weak entwining structure and let $\mathscr{A}=\operatorname{Im} \overline{p_{R}}$ and $\mathscr{B}=\operatorname{Im} \overline{p_{L}}$ be the corresponding $C$-rings. Then the restrictions of the entwining maps

$$
\psi_{L}: \mathscr{B} \rightarrow \mathscr{A}, \quad \psi_{R}: \mathscr{A} \rightarrow \mathscr{B}
$$

are inverse isomorphisms of C-rings.

Proof. Since $\overline{p_{R}}$ and $\overline{p_{L}}$ are projections, the conditions (b*) in Remark 3.4.9 imply that the restrictions of $\psi_{R}$ and $\psi_{L}$ to $\operatorname{Im} \overline{p_{R}}$ and $\operatorname{Im} \overline{p_{L}}$ respectively, are mutually inverse isomorphisms of vector spaces. Using (2.18) and the definitions of the $C$-coactions on $\mathscr{A}$ and $\mathscr{B}$, we can compute that

$$
\begin{aligned}
\left(\psi_{R} \otimes C\right) \circ \rho^{\mathscr{A}} \circ \overline{p_{R}} & =\left(\psi_{R} \otimes C\right) \circ\left(C \otimes \psi_{R}\right) \circ(\Delta \otimes A) \circ \overline{p_{R}} \\
& =(A \otimes \Delta) \circ \psi_{R} \circ \overline{p_{R}}=\rho^{\mathscr{B}} \circ \psi_{R} \circ \overline{p_{R}},
\end{aligned}
$$

therefore $\psi_{R}: \mathscr{A} \rightarrow \mathscr{B}$ is right $C$-colinear. Similarly one easily finds

$$
\begin{aligned}
\mathscr{B}_{\rho} \circ \psi_{R} \circ \overline{p_{R}} & =\left(\psi_{L} \otimes C\right) \circ(A \otimes \Delta) \circ \psi_{R} \circ \overline{p_{R}} \\
& =\left(\psi_{L} \otimes C\right) \circ\left(\psi_{R} \otimes C\right) \circ\left(C \otimes \psi_{R}\right) \circ\left(\Delta_{C} \otimes A\right) \circ \overline{p_{R}} \\
& =\left(\overline{p_{R}} \otimes C\right) \circ \rho^{\mathscr{A}} \circ \overline{p_{R}}=\rho^{\mathscr{A}} \circ \overline{p_{R}}=\left(C \otimes \psi_{R}\right) \circ \mathscr{A}^{\mathscr{A}} \circ \overline{p_{R}},
\end{aligned}
$$

where the first equality follows by the definition of ${ }^{\mathscr{B}} \rho$, the second by $(2.18)$ and the third by condition ( $\mathrm{b}^{*}$ ) of Remark 3.4.9. Then the fourth equality follows since $\overline{p_{R}}$ is a projection with image $\mathscr{A}$. Finally, the last equality holds by the definitions of ${ }^{\mathscr{A}} \rho$ and $\rho^{\mathscr{A}}$. In view of 
this calculation we conclude $\psi_{R}: \mathscr{A} \rightarrow \mathscr{B}$ is a left $C$-colinear map. By performing similar calculations it is straight forward to check that $\psi_{L}: \mathscr{B} \rightarrow \mathscr{A}$ is a $(C, C)$-bicomodule map.

We now show that $\psi_{R}: \mathscr{A} \rightarrow \mathscr{B}$ is unital and multiplicative. Firstly for the unitality take any $c \in C$ and compute

$$
\begin{aligned}
\psi_{R} \circ \eta_{\mathscr{A}}(c) & =\psi_{R} \circ \overline{p_{R}}(c \otimes 1)=\sum_{\alpha} 1_{\alpha} \otimes c^{\alpha} \\
& =\sum_{\alpha} \varepsilon\left(c_{(1)}^{\alpha}\right) 1_{\alpha} \otimes c_{(2)}=\sum_{E} \varepsilon\left(c_{(1) E}\right) 1^{E} \otimes c_{(2)} \\
& =\overline{p_{L}}(1 \otimes c)=\eta_{\mathscr{B}}
\end{aligned}
$$

where the second equality follows by (3.22), the third by (2.19) and the fourth by (2.24). Hence $\psi_{R}$ is a unital map. As for the multiplicativity, firstly observe that all elements

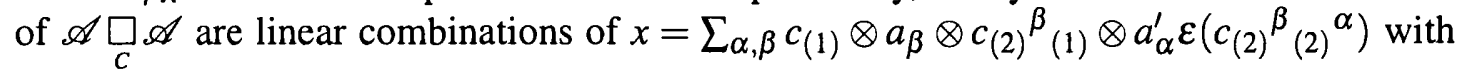
$a, a^{\prime} \in A$ and $c \in C$. For such elements the definition of the product in $\mathscr{A}$ and properties (2.16) and (3.22) yield

$$
\psi_{R} \circ \mu_{\mathscr{A}}(x)=\sum_{\alpha, \beta} \psi_{R}\left(c_{(1)} \otimes a_{\beta} a_{\alpha}^{\prime} \varepsilon\left(c_{(2)}^{\beta \alpha}\right)\right)=\psi_{R} \circ \overline{p_{R}}\left(c \otimes a a^{\prime}\right)=\psi_{R}\left(c \otimes a a^{\prime}\right) .
$$

On the other hand using (3.22) along with conditions (2.16) and (2.18), we compute

$$
\begin{aligned}
& \mu_{\mathscr{B}} \circ\left(\psi_{R} \square \psi_{R}\right)(x)=\sum_{\alpha} \mu_{\mathscr{B}} \circ\left(\psi_{R} \square \psi_{R}\right)\left(c_{(1)} \otimes a_{\alpha} \otimes \overline{p_{R}}\left(c_{(2)}{ }^{\alpha} \otimes a^{\prime}\right)\right) \\
& =\sum_{\alpha, \beta, \gamma} a_{\alpha \beta} a_{\gamma}^{\prime} \varepsilon\left(c_{(1)}^{\beta}\right) \otimes c_{(2)}^{\alpha \gamma}=\psi_{R}\left(c \otimes a a^{\prime}\right) .
\end{aligned}
$$

Therefore we conclude that $\psi_{R}$ is multiplicative, completing the proof that $\psi_{R}$ is an isomorphism of $C$-rings.

Corollary 3.4.12. Let $\left(A, C, \psi_{R}, \psi_{L}\right)$ be an invertible weak entwining structure. If $C \in$ $\mathbf{M}\left(\psi_{R}\right){ }_{A}^{C}$, then $C \in{ }_{A}^{C} \mathbf{M}\left(\psi_{L}\right)$ with the action, for all $a \in A, c \in C$,

$$
a c=\sum_{E} c_{E(1)} \varepsilon\left(c_{E(2)} a^{E}\right)
$$

Proof. We arrive at this result by making the following chain of deductions. Firstly, $C \in \mathbf{M}\left(\psi_{R}\right)_{A}^{C}$ so by Theorem 2.2.9, $C \in \mathbf{M}_{\mathscr{A}}$. Explicitly (3.17) tells us that the corresponding right $\mathscr{A}$-action is, for all $c \otimes a \in \mathscr{A}$ (summation suppressed for clarity) and $c^{\prime} \in C$,

$$
c^{\prime} \triangleleft(c \otimes a)=\varepsilon(c) c^{\prime} a .
$$

Since there is always a trivial matrix context $\left(C, C,{ }^{C} C^{C},{ }^{C} C^{C}, \sigma, \tau\right)$, as described in Proposition 3.3.14, Lemma 3.3.1 tell us that $C \in \mathscr{A} \mathbf{M}$ with the left $\mathscr{A}$-action

$$
(c \otimes a) \triangleright c^{\prime}=\sum c_{(1)} \varepsilon\left(c_{(2)} a\right) \varepsilon\left(c^{\prime}\right) .
$$

By Proposition 3.4.11, $\psi_{L}: \mathscr{B} \rightarrow \mathscr{A}$ is an isomorphism of $C$-rings and so $C \in \mathscr{B}$ M with left $\mathscr{B}$-action

$$
(a \otimes c) \triangleright c^{\prime}=\sum_{E}\left(c_{E} \otimes a^{E}\right) \triangleright c^{\prime}=\sum_{E} c_{E(1)} \varepsilon\left(c_{E(2)} a^{E}\right) \varepsilon\left(c^{\prime}\right)
$$


Finally we can use the correspondence ${ }_{\mathscr{B}} \mathbf{M} \equiv{ }_{A}^{C} \mathbf{M}\left(\psi_{L}\right)$ (cf. Theorem 2.2.10) to view $C$ in ${ }_{A}^{C} \mathbf{M}\left(\psi_{L}\right)$. Explicitly we apply the functor $\Psi: \mathscr{B} \mathbf{M} \rightarrow{ }_{A}^{C} \mathbf{M}\left(\psi_{L}\right)$, which leaves the objects and morphisms unchanged as vector spaces but which changes the left $\mathscr{B}$-actions $\overline{M \rho}$ to a left $A$-actions $\Psi(\overline{M \rho})$ defined

$$
\Psi(\bar{M} \rho): A \otimes M \rightarrow M, \quad \Psi(\bar{M} \rho):=\bar{M} \rho \circ\left(\overline{p_{L}} \square_{C} M\right) \circ\left(A \otimes{ }^{M} \rho\right)
$$

We have already demonstrated how to associate a $C$-ring to a weak entwining structure. Now we look for purely $C$-ring theoretic conditions which allow us to determine that a $C$-ring is of the type that arises from a weak entwining structure. As a corollary of the criteria that we find, we show that the Sweedler $C$-ring corresponding to a weak $A$-Galois coextension is of this type. By expanding on the conditions we are also able to characterize $C$-rings that arise from invertible weak entwining structures.

Proposition 3.4.13. Let $A$ be an algebra and $\mathscr{A}$ be a C-ring. Suppose in addition $\mathscr{A} \in{ }^{C} \mathbf{M}_{A}$ and there exists a right $A$-linear map $\theta: \mathscr{A} \rightarrow A$ satisfying the diagram

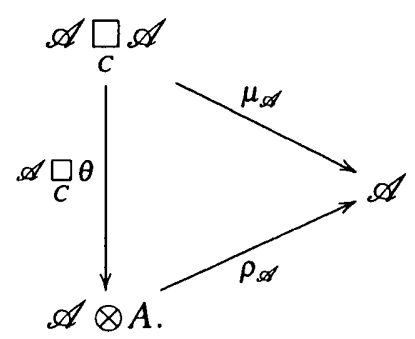

Then $\psi: C \otimes A \rightarrow A \otimes C$ defined to be the composition

$$
C \otimes A \stackrel{\eta_{\mathscr{A}} \otimes A}{\longrightarrow} \mathscr{A} \otimes A \stackrel{\rho_{\mathscr{A}}}{\longrightarrow} \mathscr{A} \stackrel{\rho^{\mathscr{A}}}{\longrightarrow} \mathscr{A} \otimes C \stackrel{\theta \otimes C}{\longrightarrow} A \otimes C
$$

makes $(A, C, \psi)$ into a weak entwining structure. Moreover the $C$-ring $\mathscr{B}$ associated to this weak entwining structure is isomorphic to $\mathscr{A}$ via the map $\chi: \mathscr{B} \rightarrow \mathscr{A}$ defined as $\chi:=\left.\rho_{\mathscr{A}} \circ\left(\eta_{\mathscr{A}} \otimes A\right)\right|_{\mathscr{B}}$.

Proof. In what follows we adopt the convention of writing $\eta_{\mathscr{A}}$ as $\eta$ and denote the right $A$-action on $\mathscr{A}$ by $\rho_{\mathscr{A}}\left(a \otimes a^{\prime}\right)=a \cdot a^{\prime}$. Before checking the required computations notice that the diagram implies the product in $\mathscr{A}$ is right $A$-linear since it is the composition of two right $A$-linear maps. To see that $\psi$ preserves the coproduct in coalgebra observe that, for all $a \in A$ and $c \in C$,

$$
\begin{aligned}
(\psi \otimes C) \circ(C \otimes \psi) \circ( & \otimes A)(c \otimes a) \\
& =\sum(\psi \otimes C)\left(c_{(1)} \otimes \theta\left(\left(\eta\left(c_{(2)}\right) \cdot a\right)_{[0]}\right) \otimes\left(\eta\left(c_{(2)}\right)_{a}\right)_{[1]}\right) \\
& =\sum(\psi \otimes C)\left(\eta(c)_{[-1]} \otimes \theta\left(\left(\eta(c)_{[0]} \cdot a\right)_{[0]}\right) \otimes\left(\eta(c)_{[0]} \cdot a\right)_{[1]}\right) \\
& =\sum(\psi \otimes C)\left((\eta(c) \cdot a)_{[-1]} \otimes \theta\left((\eta(c) \cdot a)_{[0]}\right) \otimes(\eta(c) \cdot a)_{[1]}\right) \\
& =\sum \theta\left((\eta(c) \cdot a)_{[0]}\right) \otimes(\eta(c) \cdot a)_{[1]} \otimes(\eta(c) \cdot a)_{[2]} \\
& =(A \otimes \Delta) \circ \psi(c \otimes a) .
\end{aligned}
$$


Here the second equality follows because $\eta$ is left $C$-colinear and the third by the right $A$-linearity of ${ }^{\mathscr{A}} \rho$. The penultimate equality then follows since

$$
\sum \eta\left((\eta(c) \cdot a)_{[-1]}\right) \cdot \theta\left((\eta(c) \cdot a)_{[0]}\right)=\sum \eta\left((\eta(c) \cdot a)_{[-1]}\right)(\eta(c) \cdot a)_{[0]}=\eta(c) \cdot a,
$$

by virtue of the assumption that $\mu_{\mathscr{A}}=\rho_{\mathscr{A}} \circ\left(\mathscr{A} \square \square_{c} \theta\right)$. For the preservation of the multiplication in the algebra compute, for all $a, b \in A$ and $c \in C$,

$$
\begin{aligned}
(\mu & \otimes C) \circ(A \otimes \psi) \circ(\psi \otimes A) \circ(c \otimes a \otimes b) \\
& =\sum(\mu \otimes C) \circ\left(\theta\left((\eta(c) \cdot a)_{[0]}\right) \otimes \theta\left(\left(\eta\left((\eta(c) \cdot a)_{[1]}\right) \cdot b\right)_{[0]}\right) \otimes\left(\eta\left((\eta(c) \cdot a)_{[1]}\right) \cdot b\right)_{[1]}\right) \\
& =\sum \theta\left((\eta(c) \cdot a)_{[0]}\left(\left(\eta\left((\eta(c) \cdot a)_{[1]}\right)\right) \cdot b\right)_{[0]}\right) \otimes\left(\eta\left((\eta(c) \cdot a)_{[1]}\right) \cdot b\right)_{[1]} \\
& =\sum \theta\left(\left((\eta(c) \cdot a)_{[0]} \eta\left((\eta(c) \cdot a)_{[1]}\right) \cdot b\right)_{[0]}\right) \otimes\left((\eta(c) \cdot a)_{[0]} \eta\left((\eta(c) \cdot a)_{[1]}\right) \cdot b\right)_{[1]} \\
& =\sum \theta\left((\eta(c) \cdot a b)_{[0]}\right) \otimes(\eta(c) \cdot a b)_{[1]} \\
& =\psi \circ\left(C \otimes \mu_{A}\right)(c \otimes a \otimes b) .
\end{aligned}
$$

Here the second equality follows by the diagram and the right $A$-linearity of $\theta$, and the third since product in $\mathscr{A}$ is right $C$-colinear. To show that $\psi$ respects the unit of the algebra compute, for all $c \in C$,

$$
\begin{aligned}
\sum_{\alpha} 1_{\alpha} \otimes c^{\alpha} & =\sum \theta\left((\eta(c))_{[0]}\right) \otimes(\eta(c))_{[1]} \\
& =\sum \theta\left(\eta\left(c_{(1)}\right)\right) \otimes c_{(2)} \\
& =\sum \varepsilon\left(\left(\eta\left(c_{(1)}\right)\right)_{[1]}\right) \theta\left(\left(\eta\left(c_{(1)}\right)\right)_{[0]}\right) \otimes c_{(2)} \\
& =\sum_{\alpha} \varepsilon\left(c_{(1)}^{\alpha}\right) 1_{\alpha} \otimes c_{(2)} .
\end{aligned}
$$

Here the second equality follows from the right $C$-colinearity of $\eta$. Similarly for the algebra structure one computes that, for all $a \in A$ and $c \in C$,

$$
\begin{aligned}
\sum_{\alpha} a_{\alpha} \varepsilon\left(c^{\alpha}\right) & =\sum \theta\left((\eta(c) \cdot a)_{[0]}\right) \varepsilon\left((\eta(c) \cdot a)_{[1]}\right) \\
& =\theta(\eta(c)) a \\
& =\sum_{\alpha} \varepsilon\left(c^{\alpha}\right) 1_{\alpha} a .
\end{aligned}
$$

Hence $(A, C, \psi)$ is a weak entwining structure. Now we check that $\chi:=\left.\rho_{\mathscr{A}} \circ\left(\eta_{\mathscr{A}} \otimes A\right)\right|_{\mathscr{B}}$ is morphism of $C$-rings. Firstly since it is a composition of left $C$-colinear maps it is left $C$-colinear. Then observe, for any $a \in A$ and $c \in C$,

$$
\begin{aligned}
\sum_{\alpha} \eta\left(c_{(1)}\right) \cdot a_{\alpha} \otimes c_{(2)} & \\
& =\sum \eta\left(c_{(1)}\right) \cdot \theta\left(\left(\eta\left(c_{(2)}\right) \cdot a\right)_{[0]}\right) \otimes\left(\eta\left(c_{(2)}\right) \cdot a\right)_{[1]} \\
& =\sum \eta(c)_{[0]}\left(\eta\left((\eta(c))_{[1]}\right) \cdot a\right)_{[0]} \otimes\left(\eta\left((\eta(c))_{[1]}\right) \cdot a\right)_{[1]} \\
& \left.\left.=\sum\left((\eta(c))_{[0]} \eta\left((\eta(c))_{[1]}\right) \cdot a\right)\right)_{[0]} \otimes\left((\eta(c))_{[0]} \eta\left((\eta(c))_{[1]}\right) \cdot a\right)\right)_{[1]} \\
& =\sum(\eta(c) \cdot a)_{[0]} \otimes(\eta(c) \cdot a)_{[1]}
\end{aligned}
$$


by multiple use of the properties of $\eta$, the right $A$-linearity and right $C$-colinearity of the product in $\mathscr{A}$. From this calculation it is clear that $\chi$ is right $C$-colinear. Now to show that $\chi$ is multiplicative first notice that all elements of $\mathscr{B} \square_{C} \mathscr{B}$ are linear combinations of

$$
x=\sum c_{(1)} \otimes \theta\left(\left(\eta\left(c_{(2)}\right) \cdot a\right)_{[0]}\right) \otimes\left(\eta\left(c_{(2)}\right) \cdot a\right)_{[1]} \otimes a^{\prime} \varepsilon\left(c^{\prime}\right)
$$

with $a, a^{\prime} \in A$ and $c, c^{\prime} \in C$. For such an element

$$
\begin{aligned}
\mu_{\mathscr{A}} \circ\left(\chi \square_{c} \chi\right)(x) & =\sum\left(\eta\left(c_{(1)}\right) \cdot \theta\left(\left(\eta\left(c_{(2)}\right) \cdot a\right)_{[0]}\right) \eta\left(\left(\eta\left(c_{(2)}\right) \cdot a\right)_{[1]}\right) \cdot a^{\prime} \varepsilon\left(c^{\prime}\right)\right. \\
& =\sum(\eta(c) \cdot a)_{[0]} \eta\left((\eta(c) \cdot a)_{[1]}\right) \cdot a^{\prime} \varepsilon\left(c^{\prime}\right) \\
& =(\eta(c) \cdot a) \cdot a^{\prime} \varepsilon\left(c^{\prime}\right) \\
& =\eta(c) \cdot a a^{\prime} \varepsilon\left(c^{\prime}\right)
\end{aligned}
$$

Here the second equality follows by the the same considerations employed to show the right $C$-colinearity of $\chi$. On the other hand

$$
\begin{aligned}
\chi \circ \mu_{\mathscr{B}}(x) & =\sum \chi\left(c_{(1)} \otimes \theta\left(\eta\left(c_{(2)}\right) \cdot a\right) a^{\prime} \varepsilon\left(c^{\prime}\right)\right) \\
& =\sum \eta\left(c_{(1)}\right) \cdot \theta\left(\eta\left(c_{(2)}\right) \cdot a\right) a^{\prime} \varepsilon\left(c^{\prime}\right) \\
& =\sum \eta\left((\eta(c))_{[-1]}\right) \eta(c)_{[0]} \cdot a a^{\prime} \varepsilon\left(c^{\prime}\right)=\eta(c) \cdot a a^{\prime} \varepsilon\left(c^{\prime}\right) .
\end{aligned}
$$

Here the third equality follows by the properties of $\eta$. Hence $\chi$ is multiplicative. That $\chi$ preserves the unit follows since, for all $c \in C$,

$$
\begin{aligned}
\chi \circ \eta_{\mathscr{B}}(c) & =\chi\left(c_{(1)} \otimes 1_{\alpha} \varepsilon\left(c_{(2)}^{\alpha}\right)\right)=\eta_{\mathscr{A}}\left(c_{(1)}\right) \cdot \theta\left(\eta_{\mathscr{A}}\left(c_{(2)}\right)\right) \\
& =\eta_{\mathscr{A}}\left(\left(\eta_{\mathscr{A}}(c)\right)_{[-1]}\right) \eta_{\mathscr{A}}(c)_{[0]}=\eta_{\mathscr{A}}(c)
\end{aligned}
$$

To finish the proof of the proposition we now give an inverse for $\chi$. Define

$$
\chi^{-1}: \mathscr{A} \rightarrow \mathscr{B}, \quad \chi^{-1}:=(C \otimes \theta) \circ^{\mathscr{A}} \rho .
$$

First we check $\chi^{-1}(\mathscr{A}) \subset \mathscr{B}$, recall elements of $\mathscr{B}$ are precisely the elements of $C \otimes A$ which are invariant under the map $\overline{p_{R}}:=(C \otimes A \otimes \varepsilon) \circ(C \otimes \psi) \circ(\Delta \otimes A)$ now observe, for all $a \in \mathscr{A}$,

$$
\begin{aligned}
\overline{p_{R}} \circ \chi^{-1}(a) & =\sum a_{[-1](1)} \otimes \theta\left(\left(\eta\left(a_{[-1](2)}\right) \cdot \theta\left(a_{[0]}\right)\right)\right) \\
& =\sum\left(\eta\left(a_{[-1]}\right)\right)_{[-1]} \otimes \theta\left(\left(\eta\left(a_{[-1]}\right)\right)_{[0]} a_{[0]}\right) \\
& =\sum\left(\eta\left(a_{[-1]}\right) a_{[0]}\right)_{[-1]} \otimes \theta\left(\left(\eta\left(a_{[-1]}\right) a_{[0]}\right)_{[0]}\right) \\
& =\sum a_{[-1]} \otimes \theta\left(a_{[0]}\right) \\
& =\chi^{-1}(a) .
\end{aligned}
$$

Here the second equality follows by the diagram satisfied by $\theta$ and the left $C$-colinearity of $\eta$. The third equality follows by the left $C$-colinearity of the product in $\mathscr{A}$. Now check 
that $\chi^{-1}$ as defined is indeed the inverse of $\chi$. Again compute, for all $a \in A$ and $c \in C$,

$$
\begin{aligned}
\sum_{\alpha} \chi^{-1} \circ \chi\left(c_{(1)} \otimes a_{\alpha} \varepsilon\left(c_{(2)}^{\alpha}\right)\right) & =\sum_{\alpha} \chi^{-1}\left(\eta\left(c_{(1)}\right) \cdot a_{\alpha} \varepsilon\left(c_{(2)}^{\alpha}\right)\right) \\
& =\sum_{\alpha} \eta\left(c_{(1)}\right)_{[-1]} \otimes \theta\left(\eta\left(c_{(1)}\right)_{[0]}\right) a_{\alpha} \varepsilon\left(c_{(2)}^{\alpha}\right) \\
& =\sum_{\alpha} c_{(1)} \otimes \theta\left(\eta\left(c_{(2)}\right)\right) a_{\alpha} \varepsilon\left(c_{(3)}^{\alpha}\right) \\
& =\sum c_{(1)} \otimes \theta\left(\eta\left(c_{(2)}\right)\right) \theta\left(\eta\left(c_{(3)}\right)\right) a \\
& =\sum c_{(1)} \otimes \theta\left(\eta\left(c_{(2)}\right) \eta\left(c_{(3)}\right)\right) a \\
& =\sum c_{(1)} \otimes \theta\left(\eta\left(c_{(2)}\right)\right) a \\
& =\sum c_{(1)} \otimes a_{\alpha} \varepsilon\left(c_{(2)}^{\alpha}\right) .
\end{aligned}
$$

By the right $A$-linearity of $\theta$, the assumption that $\mu_{\mathscr{A}}=\rho_{\mathscr{A}} \circ\left(\mathscr{A} \square \square_{C} \theta\right)$, the definition of $\psi$ and the properties of the map $\eta$. That $\chi \circ \chi^{-1}$ is the identity is a much simpler calculation, for all $a \in \mathscr{A}$,

$$
\chi \circ \chi^{-1}(a)=\sum \chi\left(a_{[-1]} \otimes \theta\left(a_{[0]}\right)\right)=\sum \eta\left(a_{[-1]}\right) \cdot \theta\left(a_{[0]}\right)=\sum \eta\left(a_{[-1]}\right) a_{[0]}=a .
$$

By the diagram that $\theta$ satisfies and the properties of $\eta$. This completes the proof.

Remark 3.4.14. It is easy to see that with the additional requirement that

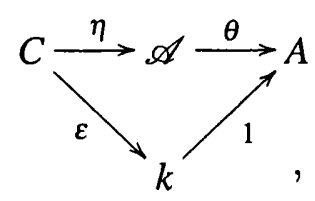

the assumptions of Proposition 3.4.13 will force $\mathscr{A}$ to be isomorphic to a $C$-ring associated to a non-weak entwining structure.

Corollary 3.4.15. Suppose $C \rightarrow B$ is a weak $A$-Galois coextension then the canonical coring $C \square_{B} C$ comes from a weak entwining structure.

Proof. By definition of $B$ we know that $C \underset{B}{\square} C \in{ }^{C} \mathbf{M}_{A}$. Also recall, from the proof of Theorem 3.4.3 there is a right $A$-linear map $\widehat{\tau}: C \square_{B} C \rightarrow A$, which we termed the cotranslation map, which is right $A$-linear. By the definition of this map it satisfies

$$
\rho_{C} \circ(C \otimes \widehat{\tau}) \circ(\Delta \otimes C)=\varepsilon \otimes C
$$

With this in mind it is clear the $\widehat{\tau}$ satisfies the diagram of the previous proposition since elements of $C \square_{B} C \square_{C} C \square_{B} C$ have the form $c^{1} \otimes c^{2}{ }_{(1)} \otimes c^{2}{ }_{(2)} \otimes c^{3}$ (summation suppressed) and so

$$
\begin{aligned}
\sum \rho_{\mathscr{A}} \circ(\mathscr{A} \otimes \widehat{\tau})\left(c^{1} \otimes c^{2}{ }_{(1)} \otimes c^{2}{ }_{(2)} \otimes c^{3}\right) & \\
& =\sum\left(C \otimes \rho_{C}\right) \circ(\mathscr{A} \otimes \widehat{\tau})\left(c^{1} \otimes c^{2}{ }_{(1)} \otimes c^{2}{ }_{(2)} \otimes c^{3}\right) \\
& =c^{1} \otimes \varepsilon\left(c^{2}\right) c^{3} .
\end{aligned}
$$


Now note this is precisely the product of these elements in $C \square_{B} C$. Hence can apply the previous proposition with $\theta=\widehat{\tau}$ to get required result.

Remark 3.4.16. From the previous corollary it is clear that the construction of a weak entwining structure from a $C$-ring in ${ }^{C} \mathbf{M}_{A}$ satisfying the conditions given in Proposition 3.4.13 generalizes the construction of a weak entwining structure from a weak $A$-Galois coextension as described in Theorem 2.2.12. In the sense that the weak entwining structure associated to a weak $A$-Galois coextension $C \rightarrow B$, as constructed in Theorem 2.2.12, can also be obtained by feeding the associated Sweedler type $C$-ring $C \square_{B} C$, which satisfies the hypotheses by the previous Corollary, into Proposition 3.4.13.

Also note that for all weak entwining structures $\left(A, C, \psi_{R}\right)$, the associated $C$-ring $\mathscr{A}$ satisfies the hypotheses of Proposition 3.4.13 when combined with the map $\varepsilon \otimes A: \mathscr{A} \rightarrow A$, and the weak entwining structure generated is $\left(A, C, \psi_{R}\right)$. Therefore all weak entwining structures can be generated in the way outlined in Proposition 3.4.13.

Corollary 3.4.17. Let $A$ be an algebra and $\mathscr{A}$ a $C$-ring. Suppose in addition that $\mathscr{A} \in$ ${ }^{C} \mathbf{M}_{A} \cap{ }_{A} \mathbf{M}^{C}$ and there exists a map $\varepsilon_{\mathscr{A}}: \mathscr{A} \rightarrow A$ which is both left and right $A$-linear satisfying the diagram

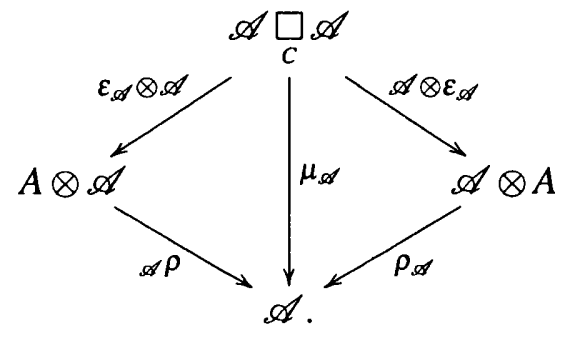

Then for the maps

$$
\begin{aligned}
& \psi_{R}: C \otimes A \rightarrow A \otimes C, \quad \psi_{R}:=\left(\varepsilon_{\mathscr{A}} \otimes C\right) \circ \rho^{\mathscr{A}} \circ \rho_{\mathscr{A}} \circ\left(\eta_{\mathscr{A}} \otimes A\right), \\
& \psi_{L}: A \otimes C \rightarrow C \otimes A, \quad \psi_{L}:=\left(C \otimes \varepsilon_{\mathscr{A}}\right) \circ^{\mathscr{A}} \rho \circ \mathscr{A} \rho \circ\left(A \otimes \eta_{\mathscr{A}}\right),
\end{aligned}
$$

$\left(A, C, \psi_{L}, \psi_{R}\right)$ is a weakly invertible entwining structure. Moreover with the additional assumption that $\mathscr{A} \in{ }_{A} \mathbf{M}_{A}, \mathscr{A}$ is also an A-coring with counit $\varepsilon_{\mathscr{A}}$ and product

$$
\Delta_{\mathscr{A}}: \mathscr{A} \rightarrow \mathscr{A} \underset{A}{\otimes \mathscr{A}}, \quad \Delta_{\mathscr{A}}(a)=\sum \eta_{\mathscr{A}}\left(a_{[-1]}\right) \underset{A}{\otimes} a_{[0]}
$$

Recall that associated to an invertible entwining structure is an object $\mathscr{B}$ which is both a $C$-ring and an A-coring. Now with this additional structure

$$
\chi: \mathscr{B} \rightarrow \mathscr{A}, \quad \chi:=\left.\rho_{\mathscr{A}} \circ\left(\eta_{\mathscr{A}} \otimes A\right)\right|_{\mathscr{B}}
$$

is an isomorphism of C-rings and A-corings.

Proof. That $\psi_{R}$ is a right-right entwining structure follows from the proposition. Similarly one can prove a left handed version of the proposition and this will imply that $\psi_{L}$ 
is a left-left entwining structure. Now with these assumptions

$$
\begin{aligned}
\overline{p_{R}} & =(C \otimes A \otimes \varepsilon) \circ\left(C \otimes \psi_{R}\right) \circ\left(\Delta_{C} \otimes A\right) \\
& =(C \otimes A \otimes \varepsilon) \circ\left(C \otimes\left(\left(\varepsilon_{\mathscr{A}} \otimes C\right) \circ \rho^{\mathscr{A}} \circ \rho_{\mathscr{A}} \circ\left(\eta_{\mathscr{A}} \otimes A\right)\right)\right) \circ\left(\Delta_{C} \otimes A\right) \\
& =\left(C \otimes \varepsilon_{\mathscr{A}}\right) \circ\left(C \otimes \rho_{\mathscr{A}}\right) \circ\left(C \otimes \eta_{\mathscr{A}} \otimes A\right) \circ\left(\Delta_{C} \otimes A\right) \\
& =\left(C \otimes \varepsilon_{\mathscr{A}}\right) \circ\left(C \otimes \rho_{\mathscr{A}}\right) \circ\left({ }^{\mathscr{A}} \rho \otimes A\right) \circ\left(\eta_{\mathscr{A}} \otimes A\right) \\
& =\left(C \otimes \varepsilon_{\mathscr{A}}\right) \circ \mathscr{A} \rho \circ \rho_{\mathscr{A}} \circ\left(\eta_{\mathscr{A}} \otimes A\right) .
\end{aligned}
$$

Here the third equality follows by the counitality of $\rho^{\mathscr{A}}$ the fourth by the left $C$-colinearity of $\eta_{\mathscr{A}}$ and final equality since the right $A$-action on $\mathscr{A}$ is left $C$-colinear. Similarly

$$
\begin{aligned}
\psi_{L} \circ \psi_{R} & =\left(C \otimes \varepsilon_{\mathscr{A}}\right) \circ \mathscr{A}^{\mathscr{A}} \rho \circ \mathscr{A} \circ\left(A \otimes \eta_{\mathscr{A}}\right) \circ\left(\varepsilon_{\mathscr{A}} \otimes C\right) \circ \rho^{\mathscr{A}} \circ \rho_{\mathscr{A}} \circ\left(\eta_{\mathscr{A}} \otimes A\right) \\
& =\left(C \otimes \varepsilon_{\mathscr{A}}\right) \circ{ }^{\mathscr{A}} \rho \circ \mathscr{A} \rho \circ\left(\varepsilon_{\mathscr{A}} \otimes \mathscr{A}\right) \circ\left(\mathscr{A} \otimes \eta_{\mathscr{A}}\right) \circ \rho^{\mathscr{A}} \circ \rho_{\mathscr{A}} \circ\left(\eta_{\mathscr{A}} \otimes A\right) \\
& =\left(C \otimes \varepsilon_{\mathscr{A}}\right) \circ \mathscr{A} \rho \circ \mu_{\mathscr{A}} \circ\left(\mathscr{A} \otimes \eta_{\mathscr{A}}\right) \circ \rho^{\mathscr{A}} \circ \rho_{\mathscr{A}} \circ\left(\eta_{\mathscr{A}} \otimes A\right) \\
& =\left(C \otimes \varepsilon_{\mathscr{A}}\right) \circ{ }^{\mathscr{A}} \rho \circ \rho_{\mathscr{A}} \circ\left(\eta_{\mathscr{A}} \otimes A\right) \\
& =\overline{p_{R}}
\end{aligned}
$$

Where the third equality follows by the diagram and the fourth by the unitality of $\eta_{\mathscr{A}}$. Likewise, one shows $\psi_{R} \circ \psi_{L}=\overline{p_{L}}$. It is immediately clear that $\chi$ is right $A$-linear. To see that it is left $\mathscr{A}$-linear observe, for all $a, a^{\prime} \in A$ and $c \in C$,

$$
\begin{aligned}
\chi\left(a^{\prime} \cdot(c \otimes a)\right) & =\sum\left(\eta_{\mathscr{A}}\left(\left(a^{\prime} \cdot \eta(c)\right)_{[-1]}\right)\right) \cdot \varepsilon_{\mathscr{A}}\left(\left(a^{\prime} \cdot \eta_{\mathscr{A}}(c)\right)_{[0]}\right) a \\
& =a^{\prime} \cdot \eta_{\mathscr{A}}(c) \cdot a \\
& =a^{\prime} \cdot \chi(c \otimes a) .
\end{aligned}
$$

Here the second equality follows by the diagram. Hence

$$
\begin{gathered}
(\underset{A}{\otimes} \chi) \circ \Delta_{\mathscr{B}} \circ \chi^{-1}(a)=\sum \eta_{\mathscr{A}}\left(a_{[-1]}\right) \underset{A}{\otimes} a_{[0]}=\Delta_{\mathscr{A}}(a), \\
\varepsilon_{\mathscr{B}} \circ \chi^{-1}=\varepsilon_{\mathscr{A}},
\end{gathered}
$$

define an $A$-coring structure on $\mathscr{A}$ which has the required properties. 


\section{Chapter 4}

\section{Structure theorems for coextensions and extensions}

\subsection{Coextensions of self-injective algebras}

In this section we investigate coalgebras which are right entwined modules over an invertible weak entwining structure. In particular we will give a criterion for such a coalgebra $C$, over a field $k$, which is a right entwined module for an invertible weak entwining structure $\left(A, C, \psi_{R}, \psi_{L}\right)$, to be weak $A$-Galois coextension. Since we will be working under the assumption $C \in \mathbf{M}\left(\psi_{R}\right)_{A}^{C}, S=E_{\mathscr{A}}(C)$ (where $\mathscr{A}$ is the $C$-ring associated to $\left(A, C, \psi_{R}\right)$ ) will be isomorphic to $B_{K}$, by the isomorphism described in the proof of Proposition 3.3.14. Moreover by Lemma 3.4.4 it is clear that $B_{\kappa}=B$, so for simplicity we shall henceforth denote all these objects by $B$.

Proposition 4.1.1. Let $\left(A, C, \psi_{R}, \psi_{L}\right)$ be an invertible weak entwining structure such that $C$ is a right entwined module, and let $\mathscr{A}$ be the $C$-ring corresponding to $\left(A, C, \psi_{R}\right)$. View $C$ as a left $A$-module as in Corollary 3.4.12. Then $C \rightarrow B$ is a weak $A$-Galois coextension and $C$ is injective as a left $B$-comodule if and only if there exists a $k$-linear map $\hat{g}: C \otimes C \rightarrow A$ such that, for all $c \in C$ and $a \in A$,

$$
\sum_{\alpha} a_{\alpha} \hat{g}\left(c^{\alpha} \otimes c^{\prime}\right)=\sum_{\alpha} \hat{g}\left(a_{\alpha} c^{\alpha} \otimes c^{\prime}\right)
$$

and

$$
\sum \hat{g}\left(c_{(1)} \otimes c_{(2)} a\right)=\sum_{\alpha} a_{\alpha} \varepsilon_{C}\left(c^{\alpha}\right)
$$

Since in Proposition 4.1.1 it is assumed that $C \in \mathbf{M}\left(\psi_{R}\right)_{A}^{C}$, using Theorem 3.4.1 we can deduce that $C \in \mathbf{M}_{\mathscr{A}}$ with the action given in (3.17). Therefore, combining Proposition 3.4.6 and Proposition 3.3.14 we find that $C \rightarrow B$ is a weak $A$-Galois coextension and $C$ is injective as a left $B$-comodule if and only if $C$ is a principal $\mathscr{A}$-module. In light of this observation, we set about proving Proposition 4.1 .1 by finding criteria for $C$ to be principal $\mathscr{A}$-module. By Theorem 3.3.16 this problem reduces to finding necessary and sufficient conditions for there to exist a left $\mathscr{A}$-module retraction of $\beta: \mathscr{A} \rightarrow C \otimes C$ (as defined in 
Proposition 3.3.6), where the right $\mathscr{A}$-module structure on $C \otimes C$ is that induced in the obvious way from the left $\mathscr{A}$-action on $C$ given by Lemma 3.3.1. Explicitly

$$
\overline{C \otimes C} \rho: \mathscr{A} \otimes C \simeq \mathscr{A} \square_{C} C \otimes C \rightarrow C \otimes C, \quad c \otimes a \otimes c^{\prime} \mapsto \sum c_{(1)} \varepsilon_{C}\left(c_{(2)} a\right) \otimes c^{\prime} .
$$

Before considering the problem of when there is a left $\mathscr{A}$-module retraction of $\beta$, we first study left $\mathscr{A}$-linear maps $C \otimes C \rightarrow \mathscr{A}$.

Lemma 4.1.2. Suppose $\left(A, C, \psi_{R}, \psi_{L}\right)$ is an invertible weak entwining structure with $C \in$ $\mathbf{M}\left(\psi_{R}\right)_{A}^{C}$. Then there is a bijective correspondence between left $\mathscr{A}$-linear maps $g: C \otimes C \rightarrow$ $\mathscr{A}$ and $k$-linear maps $\hat{g}: C \otimes C \rightarrow A$ satisfying condition (4.1).

Proof. Substituting the definition of the left $A$-action in Corollary 3.4.12 the condition 4.1 becomes

$$
\sum_{\alpha} a_{\alpha} \hat{g}\left(c^{\alpha} \otimes c^{\prime}\right)=\sum \hat{g}\left(c_{(1)} \otimes c^{\prime}\right) \varepsilon_{C}\left(c_{(2)} a\right) .
$$

Given a $k$-linear map $\hat{g}$ satisfying 4.1 we define

$$
g: C \otimes C \rightarrow \mathscr{A}, \quad g:=\overline{p_{R}} \circ(C \otimes \hat{g}) \circ\left(\Delta_{C} \otimes C\right),
$$

so on elements $g\left(d \otimes d^{\prime}\right)=\sum_{\alpha} d_{(1)} \otimes \hat{g}\left(d_{(3)} \otimes d^{\prime}\right)_{\alpha} \varepsilon_{C}\left(d_{(2)}{ }^{\alpha}\right)$. Using (2.17), (2.19) and condition (4.3), we find that, for all $d, d^{\prime} \in C$,

$$
\sum_{\alpha} \hat{g}\left(d_{(2)} \otimes d^{\prime}\right)_{\alpha} \varepsilon_{C}\left(d_{(1)}^{\alpha}\right)=\sum_{\alpha} \varepsilon_{C}\left(d_{(1)}^{\alpha}\right) 1_{\alpha} \hat{g}\left(d_{(2)} \otimes d^{\prime}\right)=\sum_{\alpha} 1_{\alpha} \hat{g}\left(d^{\alpha} \otimes d^{\prime}\right)=\hat{g}\left(d \otimes d^{\prime}\right)
$$

hence

$$
g\left(d \otimes d^{\prime}\right)=\sum d_{(1)} \otimes \hat{g}\left(d_{(2)} \otimes d^{\prime}\right) .
$$

Now observe that all elements of $\mathscr{A} \square C \otimes C$ are linear combinations of

$$
x=\sum_{\alpha} c_{(1)} \otimes a_{\alpha} \otimes c_{(2)}^{\alpha} \otimes d,
$$

with $a \in A$ and $c, d \in C$, and for such elements

$$
\begin{aligned}
g \circ \overline{c \otimes C}(x) & =\sum_{\alpha} g\left(\left(c_{(1)} \otimes a_{\alpha}\right) \triangleright\left(c_{(2)}^{\alpha} \otimes d\right)\right) \\
& =\sum_{\alpha} g\left(c_{(1)} \varepsilon_{C}\left(c_{(2)} a_{\alpha}\right) \varepsilon_{C}\left(c_{(3)}{ }^{\alpha}\right) \otimes d\right) \\
& =\sum_{c} c_{(1)} \otimes \hat{g}\left(c_{(2)} \otimes d\right) \varepsilon_{C}\left(c_{(3)} a\right) \\
& =\sum_{\alpha} c_{(1)} \otimes a_{\alpha} \hat{g}\left(c_{(2)}{ }^{\alpha} \otimes d\right) \\
& =\sum_{\alpha} c_{(1)} \otimes a_{\alpha}\left(\left(\varepsilon_{C} \otimes A\right) \circ g\left(c_{(2)}^{\alpha} \otimes d\right)\right) \\
& =\mu_{\mathscr{A}} \circ\left(\mathscr{A} \square_{C} g\right)(x),
\end{aligned}
$$

where the third equality follows by the assumption that $C \in \mathbf{M}\left(\psi_{R}\right)_{A}^{C}$ and the fourth by condition 4.3. For the fifth equality apply $\left(\varepsilon_{C} \otimes C\right)$ to (4.5), to see that $\hat{g}=\left(\varepsilon_{C} \otimes A\right) \circ g$. 
The final equality is then clear from the definition of $\mu_{\mathscr{A}}$. Therefore $g$ is a left $\mathscr{A}$-linear map.

For the converse, given a left $\mathscr{A}$-linear map $g: C \otimes C \rightarrow \mathscr{A}$ define

$$
\hat{g}: C \otimes C \rightarrow A, \quad \hat{g}:=\left(\varepsilon_{C} \otimes A\right) \circ g .
$$

Then observe that for all $d, d^{\prime} \in C$ and $a \in A$

$$
y=\sum_{\alpha} d_{(1)} \otimes a_{\alpha} \otimes d_{(2)}^{\alpha} \otimes d^{\prime}=\rho^{\mathscr{A}} \circ \overline{p_{R}}(d \otimes a) \otimes d^{\prime} \in \mathscr{A} \square C \underset{C}{\square} \otimes C .
$$

Therefore since $g$ is left $\mathscr{A}$-linear we know that $\mu_{\mathscr{A}} \circ\left(\mathscr{A} \square_{C} g\right)(y)=g \circ \overline{C \otimes C} \rho(y)$, hence

$$
\sum_{\alpha}\left(d_{(1)} \otimes a_{\alpha}\right) g\left(d_{(2)}^{\alpha} \otimes d^{\prime}\right)=\sum_{\alpha} g\left(\left(d_{(1)} \otimes a_{\alpha}\right) \triangleright\left(d_{(2)}^{\alpha} \otimes d^{\prime}\right)\right) .
$$

Finally after applying $\varepsilon_{C} \otimes A: \mathscr{A} \rightarrow A$ to the above equation, (4.7), and using the fact that it is multiplicative, we can continue to compute

$$
\begin{aligned}
\sum_{\alpha} a_{\alpha} \hat{g}\left(d^{\alpha} \otimes d^{\prime}\right) & =\sum_{\alpha} \hat{g}\left(\left(d_{(1)} \otimes a_{\alpha}\right) \triangleright\left(d_{(2)}^{\alpha} \otimes d^{\prime}\right)\right) \\
& =\sum_{\alpha} \hat{g}\left(d_{(1)} \varepsilon_{C}\left(d_{(2)} a_{\alpha}\right) \varepsilon_{C}\left(d_{(3)}{ }^{\alpha}\right) \otimes d^{\prime}\right) \\
& =\sum_{\alpha} \hat{g}\left(a_{\alpha} d^{\alpha} \otimes d^{\prime}\right)
\end{aligned}
$$

where the second equality follows by the definition of $\overline{C \otimes C} \rho$ and the third by the definition of $C \rho$, observing that since $\psi_{L} \circ \psi_{R}=\overline{p_{R}}$

$$
\sum_{\alpha} a_{\alpha} d^{\alpha}=\sum_{\alpha, E} \varepsilon_{C}\left(d^{\alpha}{ }_{E(2)} a_{\alpha}^{E}\right) d_{E(1)}^{\alpha}=\sum_{\alpha} d_{(1)} \varepsilon_{C}\left(d_{(2)} a_{\alpha}\right) \varepsilon_{C}\left(d_{(3)}^{\alpha}\right) .
$$

It remains to show that the given correspondence is one-to-one. Clearly, applying $\varepsilon_{C} \otimes A$ to $g$ given in terms of $\hat{g}$ via equation (4.5), one obtains back $\hat{g}$. On the other hand, since $g$ is left $C$-colinear, $g=\left(C \otimes \varepsilon_{C} \otimes A\right) \circ(C \otimes g) \circ\left(\Delta_{C} \otimes C\right)$, thus establishing the converse correspondence.

From the previous lemma we have established a correspondence between left $\mathscr{A}$-linear maps $g: C \otimes C \rightarrow \mathscr{A}$ and $k$-linear maps $\hat{g}: C \otimes C \rightarrow A$ satisfying (4.1). We now give a proof of Proposition 4.1 .1 by showing that the maps $\hat{g}: C \otimes C \rightarrow A$ which give left $\mathscr{A}$-linear retractions of $\beta$, under the correspondence given in (4.4), are precisely those satisfying the hypotheses of Proposition 4.1.1, i.e. conditions (4.1) and (4.2).

Proof. (of Proposition 4.1.1) Suppose that there is a map $\hat{g}: C \otimes C \rightarrow A$ satisfying (4.1) and (4.2). Then the corresponding map $g: C \otimes C \rightarrow A$, as defined in (4.4), satisfies, for all $c \otimes a \in \mathscr{A}$ (summation suppressed),

$$
\begin{aligned}
g \circ \beta(c \otimes a) & =\sum c_{(1)} \otimes \hat{g}\left(c_{(2)} \otimes c_{(3)} a\right)=\sum_{\alpha} c_{(1)} \otimes a_{\alpha} \varepsilon_{C}\left(c_{(2)}^{\alpha}\right) \\
& =\overline{p_{R}}(c \otimes a)=c \otimes a
\end{aligned}
$$


using that $\hat{g}$ satisfies condition (4.2) in the second equality and the fact that $\overline{p_{R}}$ is a projection in the final equality. Therefore $g$ is a retraction of $\beta$, hence $C \rightarrow B$ is a weak $A$-Galois coextension and $C$ is injective as a left $B$-comodule by Theorem 3.3.16.

Conversely, suppose $C \rightarrow B$ is a weak $A$-Galois coextension and $C$ is injective as a left $B$-comodule. Then, by Theorem 3.3.16 there is a left $\mathscr{A}$-module retraction $g$ of $\beta$, for which the derived map $\hat{g}:=\left(\varepsilon_{C} \otimes A\right) \circ g: C \otimes C \rightarrow A$ satisfies condition (4.3) by Lemma 4.1.2. Moreover we can compute, for all $a \in A$ and $c \in C$,

$$
\begin{aligned}
\sum \hat{g}\left(c_{(1)} \otimes c_{(2)} a\right) & =\sum_{\alpha} \hat{g}\left(c_{(1)} \otimes c_{(2)} a_{\alpha} \varepsilon_{C}\left(c_{(3)}{ }^{\alpha}\right)\right) \\
& =\left(\varepsilon_{C} \otimes A\right) \circ g \circ \beta \circ \overline{p_{R}}(c \otimes a) \\
& =\left(\varepsilon_{C} \otimes A\right) \circ \overline{p_{R}}(c \otimes a)=\sum_{\alpha} a_{\alpha} \varepsilon_{C}\left(c^{\alpha}\right),
\end{aligned}
$$

where the the first equality follows from the assumption that $C \in \mathbf{M}\left(\psi_{R}\right)_{A}^{C}$ and the final equality since $g$ is a retraction of $\beta$. Therefore $\hat{g}$ satisfies the conditions required by Proposition 4.1.1.

Remark 4.1.3. In the case where $\psi_{R}$ is a bijective map (with inverse $\psi_{L}$ ) it is clear by condition (4.3) that, for all $a \in A$ and $c, c^{\prime} \in C$,

$$
a \hat{g}\left(c \otimes c^{\prime}\right)=\sum_{\alpha, E} a_{\alpha}^{E} \hat{g}\left(c_{E}^{\alpha} \otimes c^{\prime}\right)=\sum_{\alpha, E} \hat{g}\left(a_{\alpha}^{E} c_{E}^{\alpha} \otimes c^{\prime}\right)=\hat{g}\left(a c \otimes c^{\prime}\right)
$$

therefore $\hat{g}$ is left $A$-linear. Moreover, since left linearity of $\hat{g}$ implies (4.3), it is clear that in the case of invertible entwining structures condition (4.3) is equivalent to requiring $\hat{g}$ to be left $A$-linear.

As an application of Proposition 4.1.1 we have the the following example.

Example 4.1.4. Let $H$ be a Hopf algebra with a bijective antipode $S$ and left $A$ be a right $H$-comodule subalgebra of $H$, i.e. A is a subalgebra of $H$ and $\Delta_{H}(A) \subseteq A \otimes H$. Then we can define an associated invertible entwining map

$$
\psi_{R}: H \otimes A \rightarrow A \otimes H, \quad h \otimes a \mapsto \sum a_{(1)} \otimes h a_{(2)},
$$

such that $H \in \mathbf{M}\left(\psi_{R}\right)_{A}^{C}$ and which has inverse

$$
\psi_{L}: A \otimes H \rightarrow H \otimes A, \quad a \otimes h \mapsto \sum h S^{-1}\left(a_{(2)}\right) \otimes a_{(1)} .
$$

Using the map $\psi_{L}$ it is possible to induce a left $A$-action on $H$ in the way described in Corollary 3.4.12, explicitly the action will be given by the formula $a h:=h S^{-1}(a)$. Under the additional assumption that $A$ is a direct summand of $H$ as a left $A$-module, letting $p: H \rightarrow A$ be a left $A$-linear retraction of $A \subseteq H$, we are able to define a map

$$
\hat{g}: H \otimes H \rightarrow A, \quad h \otimes h^{\prime} \mapsto p\left(S(h) h^{\prime}\right) .
$$

Using the properties of the antipode and the left $A$-linearity of $p$, we find that, for all $a \in A$ and $c, c^{\prime} \in C$,

$$
\begin{aligned}
\hat{g}\left(a h \otimes h^{\prime}\right) & =\hat{g}\left(h S^{-1}(a) \otimes h^{\prime}\right)=p\left(S\left(h S^{-1} a\right) h^{\prime}\right) \\
& =p\left(a(S h) h^{\prime}\right)=a p\left((S h) h^{\prime}\right)=a \hat{g}\left(h \otimes h^{\prime}\right)
\end{aligned}
$$


Hence $\hat{g}$ is left $A$-linear and therefore satisfies condition (4.3). Moreover, we can compute, for all $a \in A$ and $h \in H$,

$$
\sum \hat{g}\left(h_{(1)} \otimes h_{(2)} a\right)=\sum S\left(h_{(1)}\right) h_{(2)} a=\varepsilon_{H}(h) a=\sum a_{(1)} \varepsilon_{H}\left(h a_{(2)}\right)=\sum_{\alpha} a_{\alpha} \varepsilon_{H}\left(h^{\alpha}\right) .
$$

Therefore $\hat{g}$ also satisfies condition (4.2) and so, by Proposition 4.1.1, we can conclude that $H \rightarrow B$ is an $A$-Galois coextension $H$ is injective as a left $B$-comodule.

We are also able to use Proposition 4.1.1 to characterize weak Galois coextension over self-injective algebras. Recall that an algebra $A$ is called a separable algebra provided the product $\mu_{A}$ is a split epimorphism in the category of $(A, A)$-bimodules. Equivalently, $\mathrm{A}$ is a separable algebra if it has a separability element, meaning an element $e=\sum e_{1} \otimes e_{2} \in A \otimes A$ such that $\sum e_{1} e_{2}=1$ and for all $A \in A$ ae $=e a$.

Theorem 4.1.5. Let $\left(A, C, \psi_{R}, \psi_{L}\right)$ be an invertible weak entwining structure such that $C$ is a right entwined module, and let $\mathscr{A}$ be the $C$-ring associated to $\left(A, C, \psi_{R}\right)$. Suppose that the map $\beta: \mathscr{A} \rightarrow C \otimes C, c \otimes a \mapsto \sum c_{(1)} \otimes c_{(2)}$ a is injective. If $A$ is a left self-injective algebra, then $C \rightarrow B$ is a weak $A-G$ alois coextension and $C$ is injective as a left $B$-comodule. Furthermore, if $A$ is a separable algebra, then $C$ is also $A$-equivariantly injective as a left $B$-comodule (i.e., $C$ is an injective left $B$ comodule and the corresponding coaction has $a$ retraction in ${ }^{B} \mathbf{M}_{A}$ ).

Remark 4.1.6. Recall that since $k$ is assumed to be a field, $A$ is an injective $k$-module. Therefore if $A$ is a separable algebra given any two left $A$-modules $M^{\prime}$ and $M$, injective $A$ linear map $g: M^{\prime} \rightarrow M$, and $A$-linear map $f: M^{\prime} \rightarrow A$, there exist a $k$-linear map $\hat{h}: M \rightarrow H$ rendering the diagram

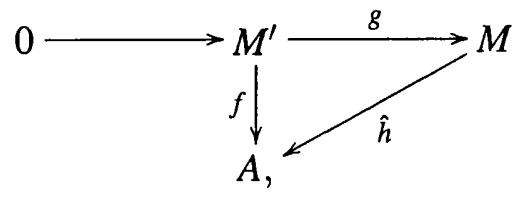

commutative. Then using a standard method for separable algebras, denoting the separability element of $A$ by $e \in A \otimes A$, define

$$
h: M \rightarrow A, \quad h:=\mu_{A} \circ(A \otimes \hat{h}) \circ\left(A \otimes_{M} \rho\right) \circ(e \otimes M),
$$

using the properties of the separability element, it is easily checked that $h$ is left $A$-linear and $h \circ g=f$. Therefore $A$ is left self injective algebra.

Proof. (of Theorem 4.1.5) Firstly, since $\mathscr{A}=\operatorname{Im} \overline{p_{R}}=\operatorname{Im} p_{L}, \mathscr{A}$ can be viewed as a left $A$-module in the way described in Theorem 2.2 .10 , explicitly by the composition

$$
A \otimes \mathscr{A} \stackrel{\psi_{L} \otimes A}{\longrightarrow} C \otimes A \otimes A \stackrel{C \otimes \mu_{A}}{\longrightarrow} \mathscr{A}
$$

Secondly, we view $C \otimes C$ as a left $A$-module via the composition

$$
A \otimes C \otimes C \stackrel{\psi_{L} \otimes C}{\longrightarrow} \mathscr{A} \otimes C \stackrel{\overline{C \otimes C \rho}}{\longrightarrow} C \otimes C
$$


which is easily seen to be equivalent to using the left $A$-action on $C$ described in Corollary 3.4.12 on the the first leg of $C \otimes C$. Next define the map

$$
r: \mathscr{A} \rightarrow A, \quad r(c \otimes a)=\varepsilon_{C}(c) a
$$

note that since the domain of this map is $\mathscr{A}$, we find that, for all $c \otimes a \in \mathscr{A}$ (summation suppressed),

$$
r(c \otimes a)=r \circ \overline{p_{R}}(c \otimes a)=\sum_{\alpha} \varepsilon_{C}\left(c^{\alpha}\right) a_{\alpha} .
$$

Moreover, for all $b \in A$ and $c \otimes a \in \mathscr{A}$ (summation suppressed)

$$
r(b(c \otimes a))=\sum_{E} \varepsilon_{C}\left(c_{E}\right) b^{E} a=\sum_{E} b \varepsilon_{C}\left(c_{E}\right) 1^{E} a=b \varepsilon_{C}(c) a
$$

where the second equality follows by (2.21) and the last equality since $c \otimes a \in \mathscr{A}$ implies that $\sum_{E} c_{E} \otimes 1^{E} a=p_{L}(c \otimes a)=c \otimes a$. Therefore we conclude that $r \in \operatorname{Hom}_{A-}(\mathscr{A}, A)$. We demonstrate that with the stated actions the map $\beta: \mathscr{A} \rightarrow C \otimes C$ is also left $A$-linear. Firstly observe that just as the corresponding left $A$-action on $C$, described in Corollary 3.4.12, is given in terms of the right $A$-action, symmetrically, it is possible to express the right $A$ action on $C$ in terms of the corresponding left $A$-action. Explicitly, using the assumptions that $C \in \mathbf{M}\left(\psi_{R}\right)_{A}^{C}$ and $\psi_{L} \circ \psi_{R}=\overline{p_{R}}$ we find that, for all $a \in A$ and $C \in C$,

$$
\begin{aligned}
\sum_{\alpha} \varepsilon_{C}\left(a_{\alpha} c^{\alpha}{ }_{(1)}\right) c^{\alpha}{ }_{(2)} & =\sum_{\alpha, E} \varepsilon_{C}\left(c^{\alpha}{ }_{(1) E} a_{\alpha}{ }^{E}\right) c^{\alpha}{ }_{(2)}=\sum_{\alpha, \beta, E} \varepsilon_{C}\left(c_{(1)}{ }_{E} a_{\alpha \beta}{ }^{E}\right) c_{(2)}{ }^{\alpha} \\
& =\sum_{\alpha, \beta} \varepsilon_{C}\left(c_{(1)} a_{\alpha \beta}\right) \varepsilon_{C}\left(c_{(2)}{ }^{\beta}\right) c_{(3)}{ }^{\alpha}=c a
\end{aligned}
$$

where the second equality follows by the definition of the left $A$-action in Corollary 3.4.12. Using the assumptions that $C \in{ }_{A}^{C} \mathbf{M}\left(\psi_{L}\right)$ and $\psi_{R} \circ \psi_{L}=\overline{p_{L}}$ we find that in particular, for all $a \in A$ and $C \in C$,

$$
\sum_{E} c_{E} a^{E}=\sum_{\alpha, E} \varepsilon_{C}\left(a^{E}{ }_{\alpha} c_{E}{ }_{(1)}\right) c_{E}{ }_{(2)}=\sum_{E} \varepsilon_{C}\left(a^{E} c_{(2)}\right) \varepsilon_{C}\left(c_{(1) E}\right) c_{(3)}=\varepsilon_{C}\left(a c_{(1)}\right) c_{(2)} .
$$

Therefore, for all $a, b \in A$ and $c \in C$,

$$
\begin{aligned}
\beta\left(b p_{L}(c \otimes a)\right) & =\sum_{E} c_{E(1)} \otimes c_{E(2)} b^{E} a=\sum_{E, F} c_{(1)} \otimes c_{(2)} b^{E F} a \\
& =\sum_{E} c_{(1)_{E}} \varepsilon_{C}\left(b^{E} c_{(2)}\right) \otimes c_{(3)} a=\sum b c_{(1)} \otimes c_{(2)} a=b \beta\left(p_{L}(c \otimes a)\right),
\end{aligned}
$$

where the second equality follows by (2.22), the third by (4.10) and the fourth since $C \in$ ${ }_{A}^{C} \mathbf{M}\left(\psi_{L}\right)$. Therefore $\beta$ is a left $A$-linear map. Furthermore, since we have assumed that $A$ is injective as a left $A$-module and $\beta$ is injective, there is an an exact sequence

$$
\operatorname{Hom}_{A-}(C \otimes C, A) \stackrel{\beta^{*}}{\longrightarrow} \operatorname{Hom}_{A-}(\mathscr{A}, A) \rightarrow 0
$$

where, for all $f \in \operatorname{Hom}_{A-}(C \otimes C, A), \beta^{*}(f)=f \circ \beta$. In view of this exact there exists $\hat{g} \in \mathrm{Hom}_{A-}(C \otimes C, A)$ s.t. $\beta^{*} \circ \hat{g}=\hat{g} \circ \beta=r$. By construction, $\hat{g}$ is left $A$-linear, hence (4.3) 
holds, and in view of (4.9) it is clear that $\hat{g}$ satisfies (4.2). Therefore by Proposition 4.1.1, $C \rightarrow B$ is a weak $A$-Galois coextension and $C$ is injective as a left $B$-comodule.

Now we prove the second part of theorem. Assume that $A$ is a separable algebra and let $e=\sum e_{1} \otimes e_{2} \in A \otimes A$ denote the separability element. Then to show that $C$ is $A$-equivariantly injective as a left $B$-comodule it suffices to show that there exists a retraction of the left $B$-coaction, given in Corollary 3.3 .4 , in ${ }^{B} \mathbf{M}_{A}$. Such a retraction can be constructed as follows. Firstly, since $C$ is injective as a left $B$-module there exists a left $B$-colinear map $\hat{\lambda}: B \otimes C \rightarrow C$ such that $\hat{\lambda} \circ{ }^{C} \rho=C$. Using this map $\hat{\lambda}$ we define

$$
\lambda: B \otimes C \rightarrow C, \quad \lambda=\rho_{C} \circ(\hat{\lambda} \otimes A) \circ\left(B \otimes \rho_{C} \otimes A\right) \circ(B \otimes C \otimes e) .
$$

Next observe that, for all $a \in A$ and $c \in C$,

$$
\begin{aligned}
{ }^{c} \rho \circ \rho_{C}(c \otimes a) & =\sum^{C} \rho\left(c_{(1)} \triangleleft\left(c_{(2)} \otimes a_{\alpha} \varepsilon_{C}\left(c_{(3)}^{\alpha}\right)\right)\right)=\sum_{\alpha} c_{(1)[-1]} \otimes c_{(1)[0]} a_{\alpha} \varepsilon_{C}\left(c_{(2)}^{\alpha}\right) \\
& =\sum_{\alpha} c_{[-1]} \otimes c_{[0](1)} a_{\alpha} \varepsilon_{C}\left(c_{[0](2)}^{\alpha}\right)=\sum c_{[-1]} \otimes c_{[0]} a \\
& =\sum_{\alpha}\left(B \otimes \rho_{C}\right) \circ\left({ }^{C} \rho \otimes A\right),
\end{aligned}
$$

in which ${ }^{C} \rho(c)=\sum c_{[-1]} \otimes c_{[0]}$ and $\overline{\rho_{C}}(c \otimes a)=c \triangleleft a$ denotes the right $\mathscr{A}$-action on $C$ given by the correspondence (3.17). The first equality in the computation holds by using the correspondence (3.17) to view the right $A$-action on $C$ as an $\mathscr{A}$-action. Then the second equality follows by using the fact that ${ }^{C} \rho$ is right $\mathscr{A}$-linear, see Corollary 3.3 .4 , and the correspondence (3.16), to return the right $\mathscr{A}$-action on $C$ back in an $A$-action. Also by the results in Corollary 3.3.4, ${ }^{C} \rho$ is right $C$-colinear hence the third equality holds. Finally the fourth equality follows from assumption that $C \in \mathbf{M}\left(\psi_{R}\right)_{A}^{C}$. Therefore $\rho_{C}$ is left $B$ colinear and it becomes clear that $\lambda$ is a composition of left $B$-colinear maps, hence is left $B$-colinear. Also observe that, for all $a \in A, b \in B$ and $c \in C$, since we know $e a=a e$, we can compute that

$$
\lambda(b \otimes c a)=\sum \hat{\lambda}\left(b \otimes c a e_{1}\right) e_{2}=\sum \hat{\lambda}\left(b \otimes c e_{1}\right) e_{2} a=\lambda(b \otimes c) a .
$$

Hence $\lambda$ is morphism in ${ }^{B} \mathbf{M}_{A}$. Next we check that $\lambda$ is a retraction from the left $B$-coaction. Again compute, for all $c \in C$,

$$
\begin{aligned}
\lambda{ }^{C} \rho(c) & =\sum \hat{\lambda}\left(c_{[-1]} \otimes c_{[0]} e_{1}\right) e_{2} \\
& =\sum \hat{\lambda}\left(\left(c e_{1}\right)_{[-1]} \otimes\left(c e_{1}\right)_{[0]}\right) e_{2} \\
& =\sum c e_{1} e_{2}=c,
\end{aligned}
$$

where the second equality follows from the left $B$-colinearity of the right $A$-action, the third because $\hat{\lambda}$ was chosen to be a splitting of the coaction and the final equality from the properties of the separability element.

To see how this result fits in with existing theorems, in the next section we derive a dual version, as it is in the setting of coalgebra-Galois extensions in which the majority of previous results have appeared. 


\subsection{Extensions of self-projective coalgebras}

In this section we start with an invertible weak entwining structure such that $A$, an algebra over a commutative ring $k$, is a right entwined module and then use Theorem 2.1.17 and Corollary 2.1.19 to deduce criteria for this algebra to be a weak $A$-Galois extension with some additional structure. Since we work in this setting, $S=$ End $^{-\mathfrak{C}}(A)$ (where $\mathfrak{C}$ is the $A$-coring associated to the (right-right) weak entwining structure) will be the same as $B=$ $A^{\text {coC }}$ (cf. Remark 2.1.14). Moreover, from the proof of Proposition 2.2.13, recall that $A^{\mathrm{coC}}=A^{\text {coC }}$, so for simplicity we shall henceforth denote all these objects by $B$.

Proposition 4.2.1. Let $\left(A, C, \psi_{R}, \psi_{L}\right)$ be an invertible weak entwining structure such that $A$ is a right entwined module, and let $\mathfrak{C}$ be the $A$-coring corresponding to $\left(A, C, \psi_{R}\right)$. View $A \otimes A$ as a left $\mathfrak{C}$-comodule via the map.

$$
A \otimes A \rho: A \otimes A \rightarrow \mathfrak{C} \otimes A, \quad a \otimes a^{\prime} \mapsto \sum a 1_{[0]} \otimes 1_{[1]} \otimes a^{\prime} .
$$

Then there exists a left $\mathfrak{C}$-colinear section of $\widetilde{c a n}_{A}: A \otimes A \rightarrow \mathfrak{C}$ if and only if there exists a $k$-linear map $\hat{f}: C \rightarrow A \otimes A$ such that, for all $c \in C$, writing $\hat{f}(c)=\sum c^{[1]} \otimes c^{[2]}$

$$
\sum c^{[1]} 1_{[0]} \otimes 1_{[1]} \otimes c^{[2]}=\sum \psi_{R}\left(c_{(1)} \otimes c_{(2)}^{[1]}\right) \otimes c_{(2)}^{[2]}
$$

and

$$
\widetilde{\operatorname{can}}_{A} \circ \hat{f}(c)=\sum_{\alpha} 1_{\alpha} \otimes c^{\alpha}
$$

Remark 4.2.2. Since in Proposition 4.2.1 it is assumed that $A \in \mathbf{M}\left(\psi_{R}\right)_{A}^{C}$, by Proposition 2.2.9A $A \mathbf{M}^{\mathfrak{C}}$, with the $\mathfrak{C}$-coaction defined, for all $a \in A$,

$$
\rho^{A}(a)=\sum_{\alpha} a_{[0]} \otimes\left(1_{\alpha} \otimes a_{[1]}^{\alpha}\right)
$$

where $\sum a_{[0]} \otimes a_{[1]}$ denotes the result of applying the right $C$-coaction on $a$, see [4, Proposition 2.3] for more details. By Example 2.1.5 since $A$ is finitely generated projective as a right $A$-module, there is a corresponding left $\mathfrak{C}$-coaction on $A$. The left $\mathfrak{C}$-coaction on $A \otimes A$ described above is the application of this left $\mathfrak{C}$-coaction on the first leg of $A \otimes A$.

Before studying when there is a left $\mathfrak{C}$-colinear retraction of $\widetilde{c a n}_{A}$, we first investigate left $\mathfrak{C}$-colinear maps $\mathfrak{C} \rightarrow A \otimes A$.

Lemma 4.2.3. Suppose $\left(A, C, \psi_{R}, \psi_{L}\right)$ is an invertible weak entwining structure with $A \in$ $\mathbf{M}\left(\psi_{R}\right)_{A}^{C}$. Then there is a bijective correspondence between left $\mathfrak{C}$-colinear maps $f: \mathfrak{C} \rightarrow$ $A \otimes A$ and $k$-linear maps $\hat{f}: C \rightarrow A \otimes A$ satisfying condition (4.13).

Proof. Since this result is a dual version of Lemma 4.1 .2 we just state the required correspondences, for a full proof see [13, Lemma 5.2]. Given a $k$-linear map $\hat{f}$ satisfying (4.13) define

$$
f: \mathfrak{C} \rightarrow A \otimes A, \quad f\left(\sum_{\alpha} a 1_{\alpha} \otimes c^{\alpha}\right)=\sum_{\alpha} a 1_{\alpha} \hat{f}\left(c^{\alpha}\right) .
$$

Conversely, given a left $\mathfrak{C}$-colinear map $f: \mathfrak{C} \rightarrow A \otimes A$ define

$$
\hat{f}: C \rightarrow A \otimes A, \quad \hat{f}(c)=f\left(\sum_{\alpha} 1_{\alpha} \otimes c^{\alpha}\right) .
$$


Then these correspondences are mutually inverse and the result holds.

We now are able to give a proof of Proposition 4.2.1 by showing that the map $\hat{f}: C \rightarrow$ $A \otimes A$ which give left $\mathfrak{C}$-colinear sections of $\widetilde{\operatorname{can}}_{A}$, under the correspondence given in 4.16, are precisely those satisfying the hypotheses of Proposition 4.2.1.

Proof. (of Proposition 4.2.1) Clearly if $f$ is a section of $\widetilde{c a n}_{A}$ then the corresponding map $\hat{f}$ satisfies (4.14). Conversely if $\hat{f}$ satisfies (4.14) then for the corresponding map $f$ we can compute that, for all $a \otimes c \in \mathfrak{C}$,

$$
\begin{aligned}
\widetilde{\operatorname{can}}_{A} \circ f(a \otimes c) & =\widetilde{\operatorname{can}}_{A}\left(\sum_{\alpha} a 1_{\alpha} \hat{f}\left(c^{\alpha}\right)\right)=\sum_{\alpha} a 1_{\alpha}\left(\widetilde{\operatorname{can}}_{A} \circ \hat{f}\left(c^{\alpha}\right)\right) \\
& =\sum_{\alpha, \beta} a 1_{\alpha} 1_{\beta} \otimes c^{\alpha \beta}=p_{R}(a \otimes c)=a \otimes c .
\end{aligned}
$$

Here the second equality follows since $\widetilde{c a n} A$ is left $A$-linear, the fourth by (2.16) and the final equality since the map $p_{R}$ leaves elements $\mathfrak{C}$ unchanged.

As motivation for the following theorem we refer the reader to [35, Theorem 3.1]. Recall that a coalgebra $C$ is called a coseparable coalgebra provided the coproduct has a retraction in the category of $C$-bicomodules. Equivalently, $C$ is a coseparable coalgebra if there exists a cointegral, i.e., a $k$-module map $\delta: C \otimes C \rightarrow k$ that is colinear, meaning, for all $c, c^{\prime} \in C$,

$$
\sum c_{(1)} \delta\left(c_{(2)} \otimes c^{\prime}\right)=\sum \delta\left(c \otimes c^{\prime}(1)\right) c^{\prime}(2),
$$

and such that $\delta \circ \Delta_{C}=\varepsilon_{C}$.

Theorem 4.2.4. Let $\left(A, C, \psi_{R}, \psi_{L}\right)$ be an invertible weak entwining structure such that $A$ is a right entwined module and let $\mathfrak{C}$ be the $A$-coring corresponding to $\left(A, C, \psi_{R}\right)$. Suppose that the map $\widetilde{c a n}_{A}: A \otimes A \rightarrow \mathfrak{C}, a \otimes a^{\prime} \mapsto a \rho^{A}\left(a^{\prime}\right)$ is surjective. If $C$ is a left self-projective $k$-flat coalgebra and the map

$$
B \otimes A \rightarrow \operatorname{Hom}^{\mathfrak{C}-}(A, A \otimes A), \quad b \otimes a \mapsto\left[a^{\prime} \mapsto a^{\prime} b \otimes a\right]
$$

is an isomorphism of left $B$-modules, then $B \subseteq A$ is weak $C$-Galois coextension and $A$ is $k$-relatively projective as a left $B$-module. Furthermore, if $C$ is a $k$-projective coseparable coalgebra and $A$ is $k$-projective, then $A$ is $C$-equivariantly projective as a left $B$-module.

Remark 4.2.5. By dualising the arguments of Remark 4.1.6, it easily shown that a $k$ projective coseparable coalgebra is left self-projective. Also note that assuming $C$ is $k$ projective implies that it is $k$-flat.

Proof. (of Theorem 4.2.4) Again, other than using some additional assumptions, the proof of this theorem is a dual version of Theorem 4.1.5, so we only provide a brief account. For more detail we refer the reader to [13, Section 6 and 7].

Firstly, observe that since $\mathfrak{C}=\operatorname{Im} p_{R}=\operatorname{Im} \overline{p_{L}}, \mathfrak{C}$ can be viewed as al left $C$-comodule in the way described in Theorem 3.4.7, explicitly ${ }^{\mathscr{B}} \rho:=\left(A \otimes \psi_{L}\right) \circ\left(A \otimes \Delta_{C}\right)$. Secondly we view $A \otimes A$ as a left $C$ comodule through the composition $\left(\psi_{L} \otimes A\right) \circ{ }^{A \otimes A} \rho$, which is easily 
seen to be equivalent to using the left $C$-coaction on $A$ described in Corollary 2.3.8 on the first leg of $A \otimes A$. Now define the map

$$
\ell: C \rightarrow \mathfrak{C}, \quad c \mapsto \sum_{\alpha} 1_{\alpha} \otimes c^{\alpha}
$$

Now observe it can be shown that $\widetilde{c a n}_{A}: A \otimes A \rightarrow \mathfrak{C}$ is left $C$-colinear and also that $\ell: C \rightarrow \mathfrak{C}$ is left $C$-colinear. Therefore since $\widetilde{c a n}_{A}$ is assumed to be surjective and $C$ to be left self projective, the

$$
\operatorname{Hom}^{C-}(C, A \otimes A) \stackrel{\operatorname{Hom}^{C-}\left(C, \widetilde{\operatorname{can}}_{A}\right)}{\longrightarrow} \operatorname{Hom}^{C-}(C, \mathfrak{C})
$$

is surjective. This implies that there exists $\hat{f} \in \operatorname{Hom}^{C-}(C, A \otimes A)$ such that

$$
\widetilde{\operatorname{can}}_{A} \circ \hat{f}=\ell \text {. }
$$

By Proposition 4.2.1 this guarantees that $\widetilde{\widetilde{c a n}_{A}}: A \otimes A \rightarrow \mathfrak{C}$ is a split epimorphism of left $\mathfrak{C}$ comodules. Therefore we can conclude from Theorem 2.1.17 that $A$ is a Galois comodule and $A$ is a $k$-relatively projective left $B$-module.

For the second part of the theorem, observe that under the more stringent condition that $A$ is $k$-projective, by Corollary 2.1.19 we know that $A$ is projective as a left $B$-module, guaranteeing the existence of a left $B$-linear map $\hat{\zeta}: A \rightarrow B \otimes A$ such that ${ }_{A} \rho \circ \hat{\zeta}=A$. Combining this with the cointegral $\delta: C \otimes C \rightarrow k$ we construct

$$
\varsigma: A \rightarrow B \otimes A, \quad \zeta=(B \otimes A \otimes \delta) \circ\left(B \otimes \rho^{A} \otimes C\right) \circ(\tilde{\zeta} \otimes C) \circ \rho^{A} .
$$

It can then be verified that this map is a section of the multiplication map in ${ }_{B} \mathbf{M}^{C}$, therefore $A$ is $C$-equivariantly projective as a left $B$-module.

Now for a Hopf algebra $H$, with bijective antipode, we can then use this theorem to produced structure theorems for certain weak $H$-Galois extensions of $H$-comodule algebras. From the first part of the theorem, assuming that $k$ is a field, we are lead to weak Hopf algebra version of the Kreimer-Takeuchi theorem [29, Theorem 1.7].

Corollary 4.2.6. Let $k$ be a field and let $H$ be a finite dimensional weak Hopf algebra over $k$. Let $A$ be a right $H$-comodule algebra and $\mathfrak{E}$ be the A-coring associated to the corresponding right-right weak entwining map, i.e. $\psi_{R}(h \otimes a):=\sum a_{[0]} \otimes h a_{[1]}$. If

$$
\widetilde{c a n}_{A}: A \otimes A \rightarrow \mathfrak{E}, \quad a \otimes b \mapsto \sum a b_{[0]} \otimes b_{[1]}
$$

is surjective, then $B \subseteq A$ is a weak Hopf-Galois extension and $A$ is projective as a left (and right) $B$-module.

Proof. Firstly, since $H$ is assumed to be finite dimensional from [3, Theorem 2.10] $H$ must has a bijective antipode. In view of this, the right-right weak entwining structure associated to the right $H$ comodule algebra $A$ is weakly invertible (cf. Proposition 2.3.5), in the sense that it forms part of weakly invertible entwining structure. Secondly, as the dual $H^{*}$ of a finite dimensional weak Hopf algebra is a weak Hopf algebra, [3, Theorem 3.11] 
implies that $H^{*}$ is a quasi-Frobenius algebra (i.e., it is self-injective). Then by [26, Remark 1.5] $H$ is projective as both a left and right $H$-comodule. Then since $k$ is assumed to be a field, all assumptions in Theorem 4.2.4 are satisfied, and in addition $A$ is $k$-projective, so $A$ is projective as a left $B$-module. That $A$ is projective as a right $B$-module follows since it is possible to produce left handed versions of the results we have used.

Also, in view of Proposition 2.3.5, from the second part Theorem 4.2.4 it is clear that

Corollary 4.2.7. Let $H$ be a coseparable $k$-projective weak Hopf algebra with bijective antipode and let $A$ be a $k$-projective right $H$-comodule algebra. Let $A$ be a right $H$-comodule algebra and $\mathfrak{E}$ be the $A$-coring associated to the corresponding right-right weak entwining map, i.e. $\psi_{R}(h \otimes a):=\sum a_{[0]} \otimes h a_{[1]}$. If the map

$$
\widetilde{\operatorname{can}}_{A}: A \otimes A \rightarrow \mathfrak{C}, \quad a \otimes b \mapsto \sum a b_{[0]} \otimes b_{[1]}
$$

is surjective, then $B \subseteq A$ is a weak $H$-Galois extension and $A$ is $H$-equivariantly projective as a left $B$-module. 


\section{Bibliography}

[1] J.N. Alonso Álvarez, J.M. Fernández Vilaboa, R. González Rodríguez and A.B. Rodríguez Raposo, Invertible weak entwining structures and weak C-cleft extensions, Preprint (2005).

[2] G. Böhm, Doi-Hopf modules over weak Hopf algebras, Comm. Algebra 28 (2000), 4687-4698.

[3] G. Böhm, F. Nill and K. Szlachányi, Weak Hopf algebras I. Integral theory and $C^{*}$-structure, J. Algebra 221 (1999), 385-438.

[4] T. Brzeziński, The structure of corings. Induction functors, Maschke-type theorem, and Frobenius and Galois-type properties, Alg. Rep. Theory, 5 (2002), 389-410.

[5] T. Brzeziński, Galois comodules J. Alg. 290 (2006), 503-537.

[6] T. Brzeziński and P.M. Hajac, The Chern-Galois character, C. R. Aced. Sci. Paris Ser. I 338 (2004), 113-116.

[7] T. Brzeziński, On modules associated to coalgebra-Galois extensions, J. Algebra 215 (1999), 290-317.

[8] T. Brzeziński and J. Gómez-Torrecillas, On comatrix corings and bimodules. K-Theory 29 (2003), 101-115.

[9] T. Brzeziński and P.M. Hajac, Coalgebra extensions and algebra coextensions of Galois type, Comm. Algebra, 27 (1999), 1347-1367.

[10] T. Brzeziński and S. Majid, Coalgebra bundles, Comm. Math. Phys. 191 (1998), 467-492.

[11] T. Brzeziński and S. Majid, Quantum group gauge theory on quantum spaces, Comm. Math. Phys. 157 (1993), 591-638.

[12] T. Brzeziński and R.B. Turner, The Galois theory of matrix $C$-rings, Preprint arxiv:math.RA/0512049 (2005), to appear in Appl. Cat. Str.

[13] T. Brzeziński, R.B. Turner and A.P. Wrightson, The structure of weak coalgebra-Galois extensions, Preprint arXiv:math.QA/0411230 (2004), to appear in Comm. Algebra. 
[14] T. Brzeziński and R. Wisbauer, Corings and Comodules. Cambridge University Press, Cambridge (2003).

[15] S. Caenepeel and E. De Groot, Modules over weak entwining structures, AMS Contemp. Math. 267 (2000), 31-54.

[16] S. Caenepeel, E. De Groot and J. Vercruysse, Constructing infinite comatrix corings from colimites. Preprint arXiv:math.RA/05116091 (2005).

[17] S. Caenepeel, G. Militaru and S. Zhu, Frobenius and Seperable Functors for Generalized Module Categories and Nonlinear Equations. SpringerVerlag, Berlin, Heidelberg, New York (2002).

[18] S. U. Chase and S. Sweedler, Hopf algebras and Galois theory. SpringerVerlag, Berlin, Heidelberg, New York (1969).

[19] A. Connes. Noncommutative geometry. Academic Press Inc., San Diego, CA, (1994).

[20] J. Cuadra and J. Gómez-Torrecillas, Galois corings and a JacobsonBourbaki type correspondence, Preprint arXiv:math.RA/0506291 (2005).

[21] Y. Doi On the structure of relative Hopf modules, Comm. Algebra 11 (1983), 243-253.

[22] Y. Doi Unifying Hopf modules, J. Algebra 153 (1992), 373-385.

[23] L. El Kaoutit and J. Gómez-Torrecillas, Comatrix corings: Galois corings, descent theory, and a structure theorem for cosemisimple corings. Math. Z. 244 (2003), 887-906.

[24] L. El Kaoutit and J. Gómez-Torrecillas, Infinite comatrix corings. Int. Math. Res. Not. 2004:39 (2002), 2017-2037.

[25] C. Faith, Algebra II. Ring Theory, Springer, Berlin (1976).

[26] J. Gómez-Torrecillas and C. Năstăsescu, Quasi-co-Frobenius coalgebras, J. Algebra 174 (1995), 909-923.

[27] J. Gómez-Torrecillas and J. Vercruysse, Comatrix corings and Galois comodules over firm rings, Preprint arXiv:math.RA/0509106 (2005).

[28] M. Koppinen Variations on the smash product with applications to groupgraded rings, J. Pure Appl. Algebra 104 (1994), 61-80.

[29] H.F. Kreimer and M. Takeuchi, Hopf algebras and Galois extensions of an algebra, Indiana Univ. Math. J. 30 (1981), 675-692.

[30] F. Nill, Axioms for weak bialgebras, Preprint ArXiv math.QA/9805104 (1998). 
[31] D.E. Radford and J. Towbar Yetter-Drinfeld categories associated to an arbitrary algebra, J. Pure Appl. Algebra 87 (1993), 259-279.

[32] P. Schauenburg Doi-Koppinen Hopf Modules Versus Entwined Modules, New York J. Math. 6 (2000), 325-329.

[33] P. Schauenburg Hopf bigalois extensions, Comm. Algebra 24 (1996), 3797-3825.

[34] H.-J. Schneider, Principal homogeneous spaces for arbitrary Hopf algebras, Israel J. Math., 72 (1990), 167-195.

[35] P. Schauenburg and H.-J. Schneider, On generalised Hopf-Galois extensions, J. Pure Appl. Algebra 202 (2005), 168-194.

[36] M. Sweedler, Hopf Algebras. W.A. Benjamin Inc., New York, 1969.

[37] M. Sweedler, The predual theorem to the Jacobson-Bourbaki theorem, Trans. Amer. Math. Soc. 213 (1975), 391-406.

[38] M. Takeuchi, Morita theorems for categories of comodules, J. Fac. Sci. Univ. Tokyo Sect. IA Math., 24 (1977), 629-644.

[39] M. Takeuchi, Relative Hopf modules - equivalences and freeness criteria, J. Algebra 60 (1979), 452-471.

[40] K. Al-Takhman, Equivalences of comodule categories for coalgebras over rings, J. Pure Applied Algebra. 173 (2002), 245-271.

[41] D. Tambara, The coendomorphism bialgebra of an algebra, J. Fac. Sci. Univ. Tokyo Sect. IA, Math. 37 (1990), 425-456.

[42] D. N. Yetter, Quantum groups and representations of monoidal categories, Math. Proc. Camb. Phil. Soc. 108 (1990), 261-290. 